TRANSACTIONS OF THE

AMERICAN MATHEMATICAL SOCIETY

Volume 353, Number 9, Pages 3465-3509

S 0002-9947(01)02771-4

Article electronically published on April 24, 2001

\title{
GROUP COHOMOLOGY AND GAUGE EQUIVALENCE OF SOME TWISTED QUANTUM DOUBLES
}

\author{
GEOFFREY MASON AND SIU-HUNG NG
}

\begin{abstract}
We study the module category associated to the quantum double of a finite abelian group $G$ twisted by a 3-cocycle, which is known to be a braided monoidal category, and investigate the question of when two such categories are equivalent. We base our discussion on an exact sequence which interweaves the ordinary and Eilenberg-Mac Lane cohomology of $G$. Roughly speaking, this reveals that the data provided by such module categories is equivalent to (among other things) a finite quadratic space equipped with a metabolizer, and also a pair of rational lattices $L \subseteq M$ with $L$ self-dual and integral.
\end{abstract}

\section{INTRODUCTION}

The purpose of the present paper is to investigate relationships that exist between certain quasi-Hopf algebras (namely, twisted quantum doubles of a finite abelian group) and their module categories. This is closely related to aspects of group cohomology concerning the relationship of the bar complex to some other complexes defined and studied by Eilenberg and Mac Lane [Mac52, EM50a and EM50b. Although our results are purely homological and algebraic in nature, some of the motivation for studying these questions derives from connections with rational conformal field theory (CFT) and vertex operator algebras [DW90], DPR92, [MS89, [DL93]. We will thus review background motivation (although it is hardly necessary for an understanding of our main results) and then give a more detailed overview of the paper.

It is not necessary for the reader to appreciate what a vertex operator algebra (VOA) is (cf. [Bor86], [FLM88], [FHL93]), but only to understand this: VOAs have a representation theory, hence a (linear) module category, and it is important to understand the nature of this category. It is expected that the module categories $V$-Mod for a number of VOAs have a braided monoidal structur 1 . We refer the reader to JS93 for the origins of braided monoidal categories, and to HL92, HL94a, HL94b], HL95a, [HL95b and references therein for more on braided monoidal categories in VOA theory. Perhaps the simplest VOAs from this perspective are called holomorphic: they have but one simple module, namely the adjoint module $V$ itself, and all modules are completely reducible. Examples of

Received by the editors December 8, 1999 and, in revised form, July 24, 2000.

2000 Mathematics Subject Classification. Primary 57T05, 16S40, 16W30.

Research of the first author was supported by the National Science Foundation and the Regents of the University of California.

${ }^{1}$ Monoidal categories and tensor categories are synonymous for us. Generally, we follow the definitions in Kas95]. 
holomorphic VOAs include the famous Moonshine Module and VOAs attached to positive-definite, even, self-dual lattices FLM88.

It is generally a very difficult problem to establish the nature of $V$-Mod, even for relatively well-understood VOAs. However, if $V$-Mod is indeed a braided monoidal category, then reconstruction theory (cf. [HO97], Maj92) suggests that there might be a quasi-Hopf algebra $Q$ with the property that $Q$-Mod is equivalent (as braided monoidal category) to $V$-Mod. (Recall that the module category of a quasi-Hopf algebra automatically carries a braided monoidal structure, cf. Kas95, Dri90.) In this context, the work of Dijkgraaf, Pasquier and Roche [DPR92 suggests how one might be able to identify such a $Q$ in the case that $V$ is a socalled holomorphic orbifold model. This means that there is a holomorphic VOA $W$ and a finite group of automorphisms $G$ of $W$ such that $V=W^{G}$, i.e., $V$ is the sub-VOA of $G$-fixed-points. The work of Dijkgraaf, Pasquier and Roche suggests that one can take $Q$ to be a twisted quantum double $D^{\omega}(G)$ of $G$. This is a certain quasi-Hopf algebra, first constructed in [DPR92], which is a version of the Drinfeld double of the group algebra $\mathbb{C}[G]$ Dri87, but which is twisted by a certain 3-cocycle $\omega$ in $Z^{3}\left(G, \mathbb{C}^{*}\right)$. For more details see [DPR92, [Mas95], and section 2 below.

One of the problems of vertex operator algebras is that of classification. There are far too many VOAs to make the problem of classification up to isomorphism at all practical, at least for the foreseeable future. A meaningful alternative is to classify VOAs up to equivalence of their module categories. Thus we would say that two VOAs $V$ and $W$ whose module categories have braided monoidal structures are equivalent if $V$-Mod and $W$-Mod are equivalent as linear, braided monoidal categories. For example, all holomorphic VOAs are equivalent in this sense. In the present paper we are interested in the case of holomorphic orbifolds: given two holomorphic VOAs $W_{1}$ and $W_{2}$, and finite automorphism groups $G_{1}$ and $G_{2}$ of $W_{1}$ and $W_{2}$ respectively, when are the corresponding holomorphic orbifolds equivalent? After the above discussion, one is led to the following purely algebraic question:

given finite groups $G_{1}$ and $G_{2}$ and normalized 3-cocycles $\omega_{1} \in$ $Z^{3}\left(G_{1}, \mathbb{C}^{*}\right)$ and $\omega_{2} \in Z^{3}\left(G_{2}, \mathbb{C}^{*}\right)$, when are the module categories $D^{\omega_{1}}\left(G_{1}\right)$-Mod and $D^{\omega_{2}}\left(G_{2}\right)$-Mod equivalent as (linear) braided monoidal categories?

Although we have used VOAs to motivate $(*)$, the question is of interest independent of the theory of VOAs; roughly, we are studying 'multiplicative Morita equivalences'. As far as we know, the question in this generality has not been discussed. In the present paper we will limit ourselves to the case in which the relevant groups and algebras are commutative. Note that it is well-known Kas95 that a sufficient condition for the equivalence of the categories in $(*)$ is that the corresponding quasi-bialgebras are gauge equivalent. We will prove (Theorem 10.5) that this is also a necessary condition, at least when the twisted quantum doubles are commutative. In practice, however, this is not very helpful in trying to decide the answer to $(*)$ in a given case. The main results and methods of this paper show essentially that there is a method that allows one to answer $\left(^{*}\right)$ in any given case. We also establish results that give a complete answer for various classes of groups.

Let us begin by considering a finite abelian group $G$. With each normalized 3-cocycle $\omega$ in $Z^{3}\left(G, \mathbb{C}^{*}\right)$ we consider the twisted quantum double $D^{\omega}(G)$ together with its group of group-like elements $\Gamma^{\omega}(G)$. Now $\Gamma^{\omega}(G)$ is an abelian group, and we will be particularly interested in the case in which $\Gamma^{\omega}(G)$ spans $D^{\omega}(G)$; this 
happens precisely when $D^{\omega}(G)$ is isomorphic as a bialgebra to the group algebra $\mathbb{C}\left[\Gamma^{\omega}(G)\right]$, in which case $\Gamma^{\omega}(G)$ is a central extension of $G$ by its group of characters $\widehat{G}$.

$$
1 \longrightarrow \widehat{G} \longrightarrow \Gamma^{\omega}(G) \longrightarrow G \longrightarrow 1 .
$$

However, there is an essential difference between the two bialgebras which generally means that they are not isomorphic as quasi-bialgebras: the associator of $D^{\omega}(G)$ carries information about the cocycle $\omega$, whereas the associator of the group algebra is trivial and carries no cohomological information.

We call the normalized 3-cocycle $\omega$ abelian in case $\Gamma^{\omega}(G)$ spans $D^{\omega}(G)$, and denote by $Z^{3}\left(G, \mathbb{C}^{*}\right)_{a b}$, resp. $H^{3}\left(G, \mathbb{C}^{*}\right)_{a b}$ the 3 -cocycles, resp. 3-cohomology classes which are abelian in this sense. They are groups, and via (1) we get a group homomorphism

$$
\Lambda: H^{3}\left(G, \mathbb{C}^{*}\right)_{a b} \longrightarrow H_{a b}^{2}(G, \widehat{G})
$$

where the subscript $a b$ on the second cohomology group refers to abelian extensions of $G$ by $\widehat{G}$.

It transpires that many of our results hinge on a close analysis of this map. Though we have described $\Lambda$ in terms of twisted quantum doubles, it turns out to have a purely homological interpretation. This arises as follows: Eilenberg and Mac Lane in [Mac52, EM50b and EM50a described a sequence of complexes $A_{i}(G)$ associated to an abelian group $G$ with a view to computing the cohomology of the Eilenberg-Mac Lane spaces $K(G, n)$. We are only interested in the complex $A_{0}(G)$, which is just the bar complex, and the second complex $A_{1}(G)$ which contains $A_{0}(G)$ as a subcomplex. This containment yields a canonical short exact sequence of complexes

$$
0 \longrightarrow \operatorname{Hom}\left(B(G), \mathbb{C}^{*}\right) \longrightarrow \operatorname{Hom}\left(A_{1}(G), \mathbb{C}^{*}\right) \longrightarrow \operatorname{Hom}\left(A_{0}(G), \mathbb{C}^{*}\right) \longrightarrow 0
$$

$\left(B(G)\right.$ is the quotient complex $\left.A_{1}(G) / A_{0}(G)\right)$, and thereby a long exact sequence in cohomology. It turns out that the map $\Lambda$ in (2) is the restriction to $H^{3}\left(G, \mathbb{C}^{*}\right)_{a b}$ of the connecting homomorphism which maps $H^{3}\left(G, \mathbb{C}^{*}\right)$ to $H^{4}\left(B(G), \mathbb{C}^{*}\right)$. There is naturally a close connection between $\Lambda$ and the cohomology of the complex $A_{1}(G)$, which is sometimes called abelian cohomology. We observe that this latter cohomology already plays a role in [MS89] and in [JS93, and is quite essential in the work of Dong and Lepowsky DL93 on the generalized Jacobi identity satisfied by the vertex operators associated to lattice elements in a lattice VOA. But the context of these works is quite different from our own.

Our analysis of the long exact sequence also reveals a fundamental difference between groups of odd order and groups of even order; we will see that $\Lambda$ is injective if, and only if, $G$ has odd order, and that in general $\operatorname{ker} \Lambda$ is isomorphic to the group 2 $\Omega_{2}(G)$ of elements in $G$ of order at most 2. We also give a precise description of $\operatorname{Im} \Lambda$ : in the easier case of groups of odd order it is precisely the group of invariants of the duality map $\epsilon: H_{a b}^{2}(G, \widehat{G}) \longrightarrow H_{a b}^{2}(G, \widehat{G})$ which maps a short exact sequence of abelian groups to the dual short exact sequence obtained by applying the functor $\operatorname{Hom}\left(?, \mathbb{C}^{*}\right)$. In case $G$ is an abelian 2-group, it will turn out from the structure of $\operatorname{Im} \Lambda$ that the extension $\Gamma^{\omega}(G)$ is necessarily a product of an even number of cyclic factors. This and other results in the paper have consequences for the theory of VOAs and CFT that we hope to pursue elsewhere.

\footnotetext{
${ }^{2}$ For a finite abelian group $G, \Omega_{p}(G)$ is the subgroup of elements of order dividing $p$.
} 
The main consequence of our analysis of the long exact sequence is that we are able to characterize the pair $(G, \omega)$ for $\omega$ abelian in terms that are more amenable to calculation. Namely, we show that it is equivalent to the existence of a certain non-degenerate quadratic form on the abelian group $\Gamma^{\omega}(G)$, call it $q$, with the property that $q$ has a metabolizer $\widehat{G}$. In general, we say that a non-degenerate quadratic space $(E, q)$ for a finite abelian group $E$ has a metabolizer $M$, if the restriction of $q$ to $M$ vanishes identically and if the order of $E$ is the square of the order of $M$. So here we are following the definition of metabolizer in tD79] rather than the classical definition Kne70, MH73], where $M$ is also required to be a direct summand of $E$. In the case we are interested in, this only happens if the extension (1) splits, and we say that $M$ is a split metabolizer in this case. For us, metabolizers are not necessarily split and the most interesting metabolizers are not. In this context we may speak of metabolic triples $(E, q, M)$, and we can try to construct suitable Witt-type groups. We essentially carry this out when $E$ is a homogeneous (or homocyclic) abelian p-group, however, we do not use the language of Witt groups. We will see (Theorem 10.4) that the equivalence $\left(^{*}\right)$ for abelian groups $G_{1}, G_{2}$ and abelian 3-cocycles $\omega_{1}, \omega_{2}$ is the same as the equivalence of the associated quadratic spaces $\left(\Gamma^{\omega_{1}}\left(G_{1}\right), q_{1}\right)$ and $\left(\Gamma^{\omega_{2}}\left(G_{2}\right), q_{2}\right)$.

We point out that quadratic forms play a prominent role in several of the papers we have already quoted concerning abelian cohomology and/or VOAs (DL93, JS93, MS89]). But in DL93 and MS89, for example, conformal field theories more general than holomorphic orbifolds are considered, so that the existence of a metabolizer, so crucial for us, plays no role in these works.

Once the connection with quadratic forms with a metabolizer has been forged, a number of further results flow. Consider a finite abelian group $G$ together with an abelian 3-cocycle $\omega$. By analogy with the standard decomposition of $G$ into a direct product of cyclic groups, one may ask whether $D^{\omega}(G)$ is gauge equivalent to a tensor product

$$
D^{\omega}(G) \sim D^{\omega_{1}}\left(C_{1}\right) \otimes \cdots \otimes D^{\omega_{r}}\left(C_{r}\right)
$$

with each $C_{i}$ a cyclic group. One may assume that $G$ is a $p$-group for some prime $p$, in which case the question has an affirmative answer in the following cases: (a) $p$ satisfies the congruence $p \equiv 1(\bmod 4) ;(b) G$ is a homogeneous $p$-group and $p$ is odd. However, the result is generally false if $p=2$, and $p \equiv 3(\bmod 4)$, and $G$ is not homogeneous. Furthermore, if there is an equivalence of the form (3), then it is generally not unique, and the direct product $C_{1} \times \cdots \times C_{r}$ of the groups $C_{i}$ is not necessarily isomorphic to $G$. It may seem curious that one has to impose congruence conditions on $p$ in order to establish (3) for all abelian $p$-groups. The reason is that via the quadratic forms we can use the technique of Gauss sums, and odd primes which are sums of two squares are better behaved. The proof of (3) for homogeneous $p$-groups is established using Witt-group type techniques. The paper of C.T.C. Wall Wal63, which classifies the quadratic forms on finite abelian groups (at least if they are of odd order) proves to be invaluable in many of our calculations.

By a rational lattice we mean a free abelian group $M$ of finite rank together with a non-degenerate bilinear form $\langle\bullet, \bullet\rangle: M \times M \longrightarrow \mathbb{Q}$. We do not necessarily assume that $\langle\bullet, \bullet\rangle$ is positive-definite. The lattice is called integral in case it takes values in $\mathbb{Z}$, and even if, in addition, one has $\langle x, x\rangle \in 2 \mathbb{Z}$. Such lattices play a prominent role in DL93] because they naturally give rise to vertex algebras, indeed to examples of 
what Dong and Lepowsky call "abelian intertwining algebras." Via the connection with quadratic forms, we are able to relate the elements of $H^{3}\left(G, \mathbb{C}^{*}\right)_{a b}$ to certain pairs of lattices $M \subseteq L$, essentially because such a pair (together with the bilinear form $\langle\bullet, \bullet\rangle)$ yields a quadratic form on $L / M$. Moreover, a theorem of Wall Wal63. allows us to see that the existence of a metabolizer implies that the lattice $M$ is necessarily self-dual (i.e., unimodular). As we will show elsewhere, this has consequences for the theory of lattice VOAs. It also permits us to see that the following pieces of data are essentially equivalen $3^{3}$ for a finite abelian group $G$ :

(a) $[\omega] \in H^{3}\left(G, \mathbb{C}^{*}\right)_{a b}$;

(b) a metabolic triple $(E, q, \widehat{G})$;

(c) a pair of rational lattices $M \subseteq L$ with $M$ even and self-dual and $L / M=G$. We will also prove (Theorem 13.8) that if $G$ is a homogeneous abelian $p$-group of odd order, then two twisted quantum doubles $D^{\omega}(G)$ and $D^{\omega^{\prime}}(G)$ for abelian cocycles $\omega$ and $\omega^{\prime}$ are gauge equivalent if, and only if, the corresponding cohomology classes $[\omega]$ and $\left[\omega^{\prime}\right]$ are equivalent under the action of the automorphism group $\operatorname{Aut}(G)$. In some sense this result is the quintessential goal that one seeks for any abelian $p$-group $G$; but the result is false in general due to "hidden" gauge equivalences which are hard to enumerate, but which can be detected via the metabolic triples.

Finally, the connection with quadratic forms allows us to construct various kinds of dualities and symmetries (other than categorical equivalence) between the module categories in $(*)$. We give various examples in section 14, including the following: for positive integers $n, k$ and odd prime $p$, let $G_{1}$ and $G_{2}$ denote the homogeneous groups $\left(\mathbb{Z}_{p^{n}}\right)^{k}$ and $\left(\mathbb{Z}_{p^{k}}\right)^{n}$ respectively. Then there are precisely $\left(\begin{array}{c}n+k \\ k\end{array}\right)$ equivalence classes of monoidal categories both of the form $D^{\omega_{1}}\left(G_{1}\right)$-Mod and $D^{\omega_{2}}\left(G_{2}\right)$-Mod, for some $\omega_{1}$ or $\omega_{2}$ respectively. Moreover, there is a canonical bijection (duality) between these two sets of equivalence classes of monoidal categories. Each such monoidal category can be labeled by a partition, and at the level of partitions the duality is simply that which maps a partition to its dual (or conjugate) partition. Moreover, duality induces a bijection between the braided monoidal categories associated to each group.

The paper is organized as follows: we give some background in section 2, including the observation that for a finite abelian group and normalized 3-cocycle $\omega \in Z^{3}\left(G, \mathbb{C}^{*}\right)$, the object $D^{\omega}(G)$-when equipped with the trivial associator-is a semi-simple self-dual Hopf algebra which is generally neither commutative nor cocommutative. We study the group-like elements of $D^{\omega}(G)$ in section 3, giving rise to the central extension (11). After a short section 4 concerned with tensor products of twisted quantum doubles, we take up in sections 58 the structure of the long exact sequence alluded to above. Much of the resulting homological information is encoded in a 7 term exact sequence which we record in Remark 8.6 In section 9 we consider the category $D^{\omega}(G)$-Mod as a monoidal category i.e., forgetting the braiding. For example, we show (Lemma 9.2 and Theorem 9.4) that for finite abelian groups $G$ of odd order and normalized 3-cocycles $\omega_{1}$ and $\omega_{2}$ in $Z^{3}\left(G, \mathbb{C}^{*}\right)_{a b}$, the monoidal categories $D^{\omega_{1}}(G)$-Mod and $D^{\omega_{2}}(G)$-Mod are tensor equivalent if, and only if, the corresponding groups of fusion rules $\Gamma^{\omega_{1}}$ and $\Gamma^{\omega_{2}}$ are isomorphic, and that in this case each $D^{\omega_{i}}(G)$ is gauge equivalent to the group algebra $\mathbb{C}\left[\Gamma^{\omega_{i}}\right]$. This answers in the affirmative-at least for groups of odd order-a question posed in [DPR92], namely, can $D^{\omega}(G)$ be obtained by twisting a Hopf algebra? We show

${ }^{3} \mathrm{~A}$ vague phrase which will be clarified in section 11 
by example that the answer is "no" in general. In section 10 we study the connections among gauge equivalence, braided monoidal categories and quadratic forms and prove the results already mentioned. Section 11 explains the connections with lattices, while section 12 presents some results involving Gauss sums and applies them to the proof of the gauge equivalence (3) in the case $p \equiv 1(\bmod 4)$. In section 13 we consider metabolic triples $(E, q, G)$ when $G$ is a homogeneous $p$-group and-in all but name-construct the corresponding Witt-type group. This is used (Theorem 13.8) to establish both (3) in the case that $G$ is a homogeneous abelian $p$-group, and other results concerning homogeneous $p$-groups already discussed. Finally, we discuss symmetries and dualities in section 14

The authors thank Chongying Dong for useful discussions.

\section{Twisted quantum Double of A Finite Group}

Let $G$ be a finite group and $\omega: G \times G \times G \longrightarrow \mathbb{C}^{*}$ be a normalized 3-cocycle 4 . For any $x, y, g \in G$, define

$$
\begin{aligned}
& \theta_{g}(x, y)=\frac{\omega(g, x, y) \omega\left(x, y,(x y)^{-1} g x y\right)}{\omega\left(x, x^{-1} g x, y\right)} \\
& \gamma_{g}(x, y)=\frac{\omega(x, y, g) \omega\left(g, g^{-1} x g, g^{-1} y g\right)}{\omega\left(x, g, g^{-1} y g\right)} .
\end{aligned}
$$

The twisted quantum double $D^{\omega}(G)$ of $G$ with respect to $\omega$ is the quasi-triangular quasi-Hopf algebra with underlying vector space $(\mathbb{C} G)^{*} \otimes \mathbb{C} G$ and multiplication,

$$
\begin{gathered}
(e(g) \otimes x)(e(h) \otimes y)=\theta_{g}(x, y) e(g) e\left(x h x^{-1}\right) \otimes x y, \\
\Delta(e(g) \otimes x)=\sum_{h k=g} \gamma_{x}(h, k) e(h) \otimes x \otimes e(k) \otimes x, \\
\Phi=\sum_{g, h, k \in G} \omega(g, h, k)^{-1} e(g) \otimes 1 \otimes e(h) \otimes 1 \otimes e(k) \otimes 1,
\end{gathered}
$$

where $\{e(g) \mid g \in G\}$ is the dual basis of the canonical basis of $\mathbb{C} G$ (cf. DPR92]). The counit and antipode are given by

$$
\epsilon(e(g) \otimes x)=\delta_{g, 1}
$$

and

$$
S(e(g) \otimes x)=\theta_{g^{-1}}\left(x, x^{-1}\right)^{-1} \gamma_{x}\left(g, g^{-1}\right)^{-1} e\left(x^{-1} g^{-1} x\right) \otimes x^{-1}
$$

where $\delta_{g, 1}$ is the Kronecker delta. The corresponding elements $\alpha$ and $\beta$ are $1_{D^{\omega}(G)}$ and $\sum_{g \in G} \omega\left(g, g^{-1}, g\right) e(g) \otimes 1$ respectively. For the definition and more details about quasi-Hopf algebras, see [Dri90], Kas95] or [CP95]. Verification of the detail involves the following identities, which result from the 3-cocycle identity for $\omega$ :

$$
\begin{gathered}
\theta_{z}(a, b) \theta_{z}(a b, c)=\theta_{a^{-1} z a}(b, c) \theta_{z}(a, b c) \\
\theta_{y}(a, b) \theta_{z}(a, b) \gamma_{a}(y, z) \gamma_{b}\left(a^{-1} y a, a^{-1} z a\right)=\theta_{y z}(a, b) \gamma_{a b}(y, z), \\
\gamma_{z}(a, b) \gamma_{z}(a b, c) \omega\left(z^{-1} a z, z^{-1} b z, z^{-1} c z\right)=\gamma_{z}(b, c) \gamma_{z}(a, b c) \omega(a, b, c),
\end{gathered}
$$

\footnotetext{
${ }^{4}$ All cocycles will take values in a trivial $G$-module.
} 
for all $a, b, c, y, z \in G$. The universal $R$-matrix is given by

$$
R=\sum_{g, h \in G} e(g) \otimes 1 \otimes e(h) \otimes g .
$$

Remark 2.1.

(i) Any Hopf algebra $H$ can be viewed as a quasi-Hopf algebra with the trivial associator $\Phi=1_{H} \otimes 1_{H} \otimes 1_{H}$ and $\alpha=\beta=1_{H}$.

(ii) If $w$ and $w^{\prime}$ are cohomologous 3-cocycles, then $D^{\omega}(G)$ and $D^{\omega^{\prime}}(G)$ are gauge equivalent as quasi-triangular quasi-bialgebras or simply gauge equivalent (cf. [Kas95]) with the algebra isomorphism $\Theta: D^{\omega}(G) \longrightarrow D^{\omega^{\prime}}(G)$ defined by

$$
\Theta(e(g) \otimes x)=\frac{b(g, x)}{b\left(x, x^{-1} g x\right)} e(g) \otimes x
$$

and the gauge transform $F \in D^{\omega^{\prime}}(G) \otimes D^{\omega^{\prime}}(G)$ given by

$$
F=\sum_{g, h} b(g, h)^{-1} e(g) \otimes 1 \otimes e(h) \otimes 1
$$

where $\omega^{\prime}=\omega \delta b$ (cf. DPR92]).

(iii) Let $\sigma$ be a group automorphism of $G$. Then $\widehat{\sigma} \omega$ defined by

$$
\widehat{\sigma} \omega(g, h, k)=\omega\left(\sigma^{-1}(g), \sigma^{-1}(h), \sigma^{-1}(k)\right)
$$

is a 3-cocycle. Moreover, $\sigma$ induces an isomorphism $\tilde{\sigma}$ of quasi-triangular quasi-Hopf algebras from $D^{\omega}(G)$ to $D^{\sigma \omega}(G)$, namely,

$$
\tilde{\sigma}(e(g) \otimes x)=e(\sigma(g)) \otimes \sigma(x) .
$$

For $g \in G$, denote by $C_{G}(g)$ the centralizer of $g$ in $G$. One can easily see that $\theta_{g}$ is a 2-cocycle of $C_{G}(g)$ with coefficient in $\mathbb{C}^{*}$. Moreover, the map $D_{g}: \omega \mapsto \theta_{g}$ induces a group homomorphism from $H^{3}\left(G, \mathbb{C}^{*}\right)$ to $H^{2}\left(C_{G}(g), \mathbb{C}^{*}\right)$. We will denote by $H^{3}\left(G, \mathbb{C}^{*}\right)_{a b}$ the group $\bigcap_{g \in G} \operatorname{ker} D_{g}$ and $Z^{3}\left(G, \mathbb{C}^{*}\right)_{a b}$ the subgroup of normalized 3 -cocycles whose cohomology classes are in $H^{3}\left(G, \mathbb{C}^{*}\right)_{a b}$.

When $G$ is abelian, $\theta_{g}=\gamma_{g}$ and $C_{G}(g)=G$ for all $g \in G$ and we will simply write $\theta_{g}$ as $\omega_{g}$. It follows from equation (10) that we have

$$
\frac{\omega_{g}(x, y) \omega_{h}(x, y)}{\omega_{g h}(x, y)}=\frac{\omega_{x y}(g, h)}{\omega_{x}(g, h) \omega_{y}(g, h)} .
$$

Hence the map $G \longrightarrow H^{2}\left(G, \mathbb{C}^{*}\right), g \mapsto\left[\omega_{g}\right]$ is a group homomorphism. Let us denote this map by $\Omega(\omega)$. Notice that $\Omega(\omega) \in H^{1}\left(G, H^{2}\left(G, \mathbb{C}^{*}\right)\right)$ and $\Omega\left(\omega \omega^{\prime}\right)=$ $\Omega(\omega) \Omega\left(\omega^{\prime}\right)$ for any other 3-cocycle $\omega^{\prime}$. Moreover, $\Omega(\omega)$ is independent of the choice of representative of the cohomology class of $\omega$. Therefore, $\Omega$ induces a group homomorphism $\bar{\Omega}$ from $H^{3}\left(G, \mathbb{C}^{*}\right)$ to $H^{1}\left(G, H^{2}\left(G, \mathbb{C}^{*}\right)\right)$, and $H^{3}\left(G, \mathbb{C}^{*}\right)_{a b}=\operatorname{ker} \bar{\Omega}$.

Proposition 2.2. Let $G$ be a finite abelian group and $\omega$ a normalized 3-cocycle.

(i) When equipped with the trivial associator, $D^{\omega}(G)$ is a self-dual Hopf algebra.

(ii) Let $b: G \times G \longrightarrow \mathbb{C}^{*}$ be a normalized 2-cochain and $\omega^{\prime}=\omega \delta b$. Then the map $\Theta: D^{\omega}(G) \longrightarrow D^{\omega^{\prime}}(G)$ defined by

$$
\Theta(e(g) \otimes x)=\frac{b(g, x)}{b(x, g)} e(g) \otimes x
$$

is an isomorphism of Hopf algebras. 
Proof. (i) Since $G$ is abelian, $e(g) \otimes 1$ is in the center of $D^{\omega}(G)$. Hence, $\beta \in$ $\operatorname{Cent}\left(D^{\omega}(G)\right)$ and $\Phi \in \operatorname{Cent}\left(D^{\omega}(G)^{\otimes 3}\right)$. Therefore, $\Delta$ is coassociative and $S$ satisfies the antipode conditions.

To show that $D^{\omega}(G)$ is self-dual, let us denote by $\left\{f_{x, g}\right\}_{g, x \in G}$ the dual basis of $\{e(g) \otimes x\}_{g, x \in G}$. Then,

$$
\begin{aligned}
f_{x, g} * f_{y, h}(e(k) \otimes z) & =\left(f_{x, g} \otimes f_{y, h}\right)\left(\sum_{a b=k} \omega_{z}(a, b) e(a) \otimes z \otimes e(b) \otimes z\right) \\
& =\delta_{x, z} \delta_{y, z} \delta_{g h, k} \omega_{x}(g, h) \\
& =\delta_{x, y} \omega_{x}(g, h) f_{x, g h}(e(k) \otimes z) .
\end{aligned}
$$

Therefore,

$$
f_{x, g} * f_{y, h}=\delta_{x, y} \omega_{x}(g, h) f_{x, g h} .
$$

Similarly, one can derive that

$$
\Delta\left(f_{x, g}\right)=\sum_{u v=x} \omega_{g}(u, v) f_{u, g} \otimes f_{v, g} .
$$

The identity, $1_{D^{\omega}(G)^{*}}$, and the counit, $\epsilon$, of $D^{\omega}(G)^{*}$ are given by

$$
1_{D^{\omega}(G)^{*}}=\sum_{x \in G} f_{x, 1}, \quad \epsilon\left(f_{x, g}\right)=\delta_{x, 1}
$$

respectively, Therefore, the linear map $\varphi: D^{\omega}(G) \longrightarrow D^{\omega}(G)^{*}$ defined by

$$
\varphi(e(x) \otimes g)=f_{x, g}
$$

is a Hopf algebra isomorphism.

(ii) Notice that for any $x, y, g \in G$

$$
\omega_{g}^{\prime}(x, y)=\omega_{g}(x, y) \delta b_{g}(x, y)
$$

where $b_{g}(x)=\frac{b(x, g)}{b(g, x)}$. The result follows immediately from this identity.

In the sequel, we will denote by $D^{\omega}(G)_{0}$ the Hopf algebra structure on $D^{\omega}(G)$, and we use $D^{\omega}(G)$ for the quasi-Hopf algebra structure with respect to the associator $\Phi$ given by equation (8).

\section{Group-like elements of $D^{\omega}(G)$}

Definition 3.1. Let $G$ be a finite group and $\omega$ a normalized 3-cocycle. A nonzero element $u$ in $D^{\omega}(G)$ is called group-like if $\Delta(u)=u \otimes u$. We will denote by $\Gamma^{\omega}(G)$ the set of all group-like elements of $D^{\omega}(G)$. We simply write $\Gamma^{\omega}$ for $\Gamma^{\omega}(G)$ when the context is clear.

As in the case of group-like elements in a coalgebra (cf. [Swe69]), $\Gamma^{\omega}$ is a linearly independent set and any group-like element $u$ is invertible in $D^{\omega}(G)$ with inverse $S(u)$. Since $\Delta: D^{\omega}(G) \longrightarrow D^{\omega}(G) \otimes D^{\omega}(G)$ is an algebra map, $\Gamma^{\omega}$ is a subgroup of the group of units of $D^{\omega}(G)$. Moreover, $\Gamma^{\omega}$ can be characterized by the following proposition. 
Proposition 3.2. A nonzero element $u$ in $D^{\omega}(G)$ is a group-like element if, and only if, $u=\sum_{g \in G} \alpha(g) e(g) \otimes x$ for some $x \in G$ and a map $\alpha: G \longrightarrow \mathbb{C}^{*}$ such that

$$
\gamma_{x}(g, h)=\frac{\alpha(g) \alpha(h)}{\alpha(g h)}
$$

for any $g, h \in G$.

Proof. The result follows from direct computation.

Let $\widehat{G}$ be the character group of $G$. Since $\gamma_{1}=1, \sum_{g \in G} \alpha(g) e(g) \otimes 1 \in \Gamma^{\omega}$ for any $\alpha \in \widehat{G}$ by Proposition [3.2. The map $\widehat{G} \longrightarrow \Gamma^{\omega}, \alpha \mapsto \sum_{g \in G} \alpha(g) e(g) \otimes 1$ is an injective group homomorphism. In the sequel, we often identify $\widehat{G}$ with the image of $\widehat{G}$ under this map. On the other hand, the map

$$
D^{\omega}(G) \longrightarrow \mathbb{C} G, \quad \sum_{g, x \in G} \alpha(g, x) e(g) \otimes x \mapsto \sum_{x \in G} \alpha(1, x) x
$$

is an algebra map. Let $B^{\omega}$ be the image of $\Gamma^{\omega}$ under this map. One can easily see that

$$
B^{\omega}=\left\{x \in G \mid \gamma_{x} \text { is a 2-coboundary }\right\} .
$$

Lemma 3.3. Let $G$ be a finite group. Then $\widehat{G}$ is in the center of $D^{\omega}(G)$. Moreover, $\Gamma^{\omega}$ is a central extension

$$
1 \longrightarrow \widehat{G} \longrightarrow \Gamma^{\omega} \longrightarrow B^{\omega} \longrightarrow 1 \text {. }
$$

For each $x \in B^{\omega}$, let $\gamma_{x}=\delta \tau_{x}$ for a 1-cochain $\tau_{x}: G \longrightarrow \mathbb{C}^{*}$. The 2-cocycle $\beta$ associated to this central extension is given by

$$
\beta(x, y)(g)=\frac{\tau_{x}(g) \tau_{y}\left(x^{-1} g x\right)}{\tau_{x y}(g)} \theta_{g}(x, y) .
$$

Proof. For any $\alpha \in \widehat{G}$ and $e(h) \otimes x \in D^{\omega}(G)$,

$$
\begin{aligned}
(e(h) \otimes x) \cdot\left(\sum_{g \in G} \alpha(g) e(g) \otimes 1\right) & =\sum_{g \in G} \alpha(g) e(h) e\left(x^{-1} g x\right) \otimes x \\
& =\sum_{g \in G} \alpha\left(x g x^{-1}\right) e(h) e(g) \otimes x \\
& =\sum_{g \in G} \alpha(g) e(h) e(g) \otimes x \\
& =\left(\sum_{g \in G} \alpha(g) e(g) \otimes 1\right) \cdot(e(h) \otimes x) .
\end{aligned}
$$

Therefore, the first statement follows.

An element $u \in \operatorname{ker}\left(\Gamma^{\omega} \longrightarrow B^{\omega}\right)$ if, and only if, $u=\sum_{g \in G} \alpha(g) e(g) \otimes 1$ and $\gamma_{1}=\delta \alpha$.

Since $\gamma_{1}=1, \alpha \in \widehat{G}$. Hence,

$$
\widehat{G}=\operatorname{ker}\left(\Gamma^{\omega} \longrightarrow B^{\omega}\right) .
$$

Since $\gamma_{x}=\delta \tau_{x}$ for $x \in B^{\omega}$,

$$
x \mapsto \sum_{g \in G} \tau_{x}(g) e(g) \otimes x
$$


is a section of the map $\Gamma^{\omega} \longrightarrow B^{\omega}$. One can check directly that

$$
\begin{array}{r}
\left(\sum_{g \in G} \tau_{x}(g) e(g) \otimes x\right) \cdot\left(\sum_{g \in G} \tau_{y}(g) e(g) \otimes y\right) \\
=\sum_{g \in G} \tau_{x}(g) \tau_{y}\left(x^{-1} g x\right) \theta_{g}(x, y) e(g) \otimes x y .
\end{array}
$$

Hence, the formula for the 2-cocycle associated to the central extension follows.

Remark 3.4. In general, $B^{\omega}$ and the extension $\Gamma^{\omega}$ depend on the individual cocycle. However, both of them are independent of the representative of the cohomology class $[\omega]$ when $G$ is abelian.

Proposition 3.5. Let $G$ be a finite abelian group and $\omega, \omega^{\prime}$ normalized 3-cocycles of $G$.

(i) $\Gamma^{\omega}$ is abelian.

(ii) If $\omega$ and $\omega^{\prime}$ are cohomologous, then $B^{\omega}=B^{\omega^{\prime}}$ and the central extensions

$$
1 \longrightarrow \widehat{G} \longrightarrow \Gamma^{\omega} \longrightarrow B^{\omega} \longrightarrow 1 \text { and } 1 \longrightarrow \widehat{G} \longrightarrow \Gamma^{\omega^{\prime}} \longrightarrow B^{\omega^{\prime}} \longrightarrow 1
$$

are equivalent.

Proof. (i) By Proposition 2.2, $D^{\omega}(G)_{0}$ is a self-dual Hopf algebra and hence $\Gamma^{\omega}$ is isomorphic to the group of group-like elements of $D^{\omega}(G)_{0}^{*}$. Let $\sigma_{1}, \sigma_{2}$ be group-like elements of $D^{\omega}(G)_{0}^{*}$. Then, $\sigma_{1}, \sigma_{2}: D^{\omega}(G) \longrightarrow \mathbb{C}$ are algebra maps. Let $V_{1}, V_{2}$ be the 1-dimensional representations associated to $\sigma_{1}$ and $\sigma_{2}$ respectively. Then, $V_{1} \otimes V_{2}$ and $V_{2} \otimes V_{1}$ are the 1-dimensional representations associated to $\sigma_{1} * \sigma_{2}$ and $\sigma_{2} * \sigma_{1}$ respectively. Since $V_{1} \otimes V_{2} \cong V_{2} \otimes V_{1}$ as $D^{\omega}(G)$ modules, $\sigma_{1} * \sigma_{2}=\sigma_{2} * \sigma_{1}$. Therefore, $\Gamma^{\omega}$ is abelian.

(ii) Let $\omega^{\prime}=\omega \delta b$ for some normalized 2-cochain $b: G \times G \longrightarrow \mathbb{C}^{*}$. Then

$$
\omega_{g}^{\prime}(x, y)=\omega_{g}(x, y) \delta b_{g}(x, y)
$$

where $b_{g}(x)=b(x, g) / b(g, x)$. Hence, $\omega_{g}^{\prime}$ is a 2-coboundary if, and only if, $\omega_{g}$ is a 2-coboundary. Therefore, $B^{\omega^{\prime}}=B^{\omega}$. Since the map $\Theta: D^{\omega}(G)_{0} \longrightarrow D^{\omega^{\prime}}(G)_{0}$ defined in Proposition 2.2 is a Hopf algebra isomorphism, $\Theta\left(\Gamma^{\omega}\right)=\Gamma^{\omega^{\prime}}$. Moreover, $\Theta$ satisfies the commutative diagram

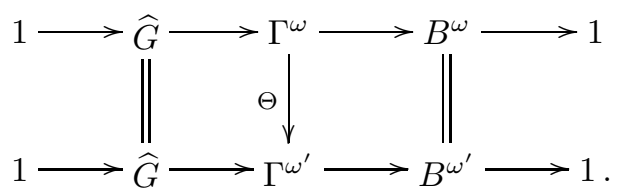

Corollary 3.6. Let $G$ be a finite group and $\omega$ a normalized 3-cocycle. Then the following statements are equivalent:

(i) $D^{\omega}(G)$ is spanned by $\Gamma^{\omega}$.

(ii) $G$ is abelian and $B^{\omega}=G$.

(iii) $G$ is abelian and $\omega \in Z^{3}\left(G, \mathbb{C}^{*}\right)_{a b}$.

(iv) $D^{\omega}(G)$ is a commutative algebra. 
Proof. (i) $\Leftrightarrow$ (ii). It follows from Lemma 3.3 that

$$
\left|\Gamma^{\omega}\right|=|\widehat{G}| \cdot\left|B^{\omega}\right| \text {. }
$$

Therefore, $D^{\omega}(G)$ is spanned by $\Gamma^{\omega}$ if and only if $|\widehat{G}|=|G|$ and $\left|B^{\omega}\right|=|G|$ which is equivalent to the statement (ii).

The equivalence of (ii) and (iii) follows directly from the definition of $Z^{3}\left(G, \mathbb{C}^{*}\right)_{a b}$ and equation (6). (ii) $\Rightarrow$ (iv). Since $G$ is abelian and $B^{\omega}=G, \omega_{g}$ is a coboundary for any $g \in G$. In particular, $\omega_{g}(x, y)=\omega_{g}(y, x)$ for any $x, y \in G$. Therefore, the multiplication on $D^{\omega}(G)$ is commutative.

(iv) $\Rightarrow$ (ii) Assume $D^{\omega}(G)$ is commutative. By the surjectivity of the algebra map defined in (14), $G$ is abelian and hence $\theta_{g}=\gamma_{g}=\omega_{g}$. Moreover, the commutative multiplication in $D^{\omega}(G)$ implies that

$$
\omega_{g}(x, y)=\omega_{g}(y, x)
$$

for any $x, y, g \in G$. Therefore, $\omega_{g}$ is a 2 -coboundary for any $g \in G$.

In the sequel, if $G$ is a finite abelian group, we will denote by $\Lambda_{G}[\omega]$ the cohomology class in $H^{2}\left(B^{\omega}, \widehat{G}\right)$ which corresponds to the central extension

$$
1 \longrightarrow \widehat{G} \longrightarrow \Gamma^{\omega} \longrightarrow B^{\omega} \longrightarrow 1
$$

In particular, if $[\omega] \in H^{3}\left(G, \mathbb{C}^{*}\right)_{a b}$, then $B^{\omega}=G$ and so $\Lambda_{G}[\omega] \in H^{2}(G, \widehat{G})$. We simply write $\Lambda$ instead of $\Lambda_{G}$ when there is no ambiguity.

Remark 3.7. If one of the conditions (i)-(iv) of Corollary 3.6 holds, then we have an isomorphism of Hopf algebras $D^{\omega}(G)_{0} \cong \mathbb{C}\left[\Gamma^{\omega}\right]$.

Proposition 3.8. The map $\Lambda: H^{3}\left(G, \mathbb{C}^{*}\right)_{a b} \longrightarrow H^{2}(G, \widehat{G}),[\omega] \mapsto \lambda[\omega]$ is a group homomorphism.

Proof. Let $[\omega] \in H^{3}\left(G, \mathbb{C}^{*}\right)_{a b}$. Then for each $x \in G$, there is a normalized 1-cochain $\tau_{x}$ such that $\delta \tau_{x}=\omega_{x}$. Then $\Lambda[\omega]$ can be represented by $\beta$ where $\beta: G \times G \longrightarrow \widehat{G}$ is given by

$$
\beta(x, y)(z)=\frac{\tau_{x}(z) \tau_{y}(z)}{\tau_{x y}(z)} \omega_{z}(x, y) .
$$

The result follows easily from this formula.

Let $G$ be a finite abelian group and $\omega \in Z^{3}\left(G, \mathbb{C}^{*}\right)_{a b}$. Denote by $T(\omega)$ the set of all normalized 2-cochains $\tau$ on $G$ such that

$$
\omega_{x}=\delta \tau_{x}
$$

for $x \in G$ where $\tau_{x}(y)=\tau(x, y)$. Pick any $\tau \in T(\omega)$. Denote by $\sigma(\alpha, x)$ the group-like element

$$
\sum_{g \in G} \alpha(g) \tau_{x}(g) e(g) \otimes x
$$

for any $\alpha \in \widehat{G}$ and $x \in G$. We will write $\sigma_{\tau}(\alpha, x)$ if we wish to emphasize the dependence of $\sigma$ on $\tau$. By Lemma 3.3. for any $u \in \Gamma^{\omega}$, there exist unique $\alpha \in \widehat{G}$ and $x \in G$ such that $u=\sigma(\alpha, x)$. It follows from Proposition 3.8 that

$$
\sigma(\alpha, x) \sigma(\lambda, y)=\sigma(\alpha \lambda \beta(x, y), x y)
$$


for any $\alpha, \lambda \in \widehat{G}$ and $x, y \in G$ where $\beta$ is given by equation (15). We will simply write $\Lambda(\omega)$ for the 2-cocycle given in equation (15) whenever the context is clear.

\section{TENSOR PRODUCTS}

We consider here the structure of the tensor product of two quantum doubles. Let $G, H$ be finite groups and $\omega, \omega^{\prime}$ normalized 3-cocycles on $G$ and $H$ respectively (with $\mathbb{C}^{*}$ coefficients as always). Of course there are canonical projections $G \times H \longrightarrow G$, $G \times H \longrightarrow H$, and we let infl $\omega$, infl $\omega^{\prime}$ denote the 3 -cocycles in $Z^{3}\left(G \times H, \mathbb{C}^{*}\right)$ obtained by inflating $\omega, \omega^{\prime}$ using these projections. Let $\zeta=($ infl $\omega)\left(\right.$ infl $\left.\omega^{\prime}\right)$.

Proposition 4.1. There is a natural isomorphism of quasi-triangular quasi-Hopf algebras

$$
\iota: D^{\omega}(G) \otimes D^{\omega^{\prime}}(H) \stackrel{\cong}{\longrightarrow} D^{\zeta}(G \times H)
$$

given by $\iota: e(g) \otimes x \otimes e(h) \otimes y \mapsto e(g, h) \otimes(x, y)$ for any $g, x \in G$ and $h, y \in H$.

This means that $\iota$ is an isomorphism of bialgebras, and that it maps the Drinfeld associators, $\mathcal{R}$-matrices and elements $\alpha, \beta$ (as given in section 21) to the corresponding objects associated with $D^{\zeta}(G \times H)$. The calculations needed to prove the proposition are essentially routine and we omit the proof.

The group-like elements in $D^{\omega}(G) \otimes D^{\omega^{\prime}}(H)$ are of the form

$$
\left(\sum_{g \in G} \lambda_{x}(g) e(g) \otimes x\right) \otimes\left(\sum_{h \in H} \lambda_{y}^{\prime}(h) e(h) \otimes y\right)
$$

where $x \in B^{\omega}, y \in B^{\omega^{\prime}}$ and $\lambda_{x}$ and $\lambda_{y}^{\prime}$ are functions on $G$ and $H$ respectively such that $\delta \lambda_{x}=\gamma_{x}, \delta \lambda_{y}^{\prime}=\gamma_{y}^{\prime}$ and $\gamma, \gamma^{\prime}$ are those $\gamma^{\prime}$ 's associated to $\omega$ and $\omega^{\prime}$ respectively (cf. (5D)). It is easy to see that the group of all group-like elements in $D^{\omega}(G) \otimes$ $D^{\omega^{\prime}}(H)$ is naturally isomorphic to $\Gamma^{\omega}(G) \times \Gamma^{\omega^{\prime}}(H)$. As $\iota$ induces an isomorphism from the group of group-like elements of $D^{\omega}(G) \otimes D^{\omega^{\prime}}(H)$ to $\Gamma^{\zeta}(G \times H)$,

$$
\Gamma^{\omega}(G) \times \Gamma^{\omega^{\prime}}(H) \stackrel{\iota}{\cong} \Gamma^{\zeta}(G \times H) .
$$

Proposition 4.2. Let $G$ be a finite group given as a direct product of groups $G=$ $H \times K$ with $|H|$ and $|K|$ coprime. Then for any normalized 3-cocycle $\omega$ of $G$, there exist normalized 3-cocycles $\eta, \eta^{\prime}$ on $K, H$ respectively such that $D^{\omega}(G)$ and $D^{\eta}(H) \otimes D^{\eta^{\prime}}(K)$ are equivalent as quasi-triangular quasi-bialgebras.

Proof. It is well-known that $H^{n}\left(G, \mathbb{C}^{*}\right)=\operatorname{infl}\left(H^{n}\left(H, \mathbb{C}^{*}\right)\right) \operatorname{infl}\left(H^{n}\left(K, \mathbb{C}^{*}\right)\right)$ (see Bro82]) (written multiplicatively), and in particular, for any normalized 3-cocycle $\omega$ of $G, \omega$ is cohomologous to $\zeta=($ infl $\eta)\left(\right.$ infl $\left.\eta^{\prime}\right)$ for some normalized 3-cocycles $\eta, \eta^{\prime}$ on $K, H$ respectively. Hence, $D^{\omega}(G)$ and $D^{\eta}(H) \otimes D^{\eta^{\prime}}(K)$ are equivalent as quasi-triangular quasi-bialgebras by Proposition 4.1 and Remark 2.1] (ii).

We state formally what obtains when $G$ is abelian since we will need this case.

Proposition 4.3. Let $G$ be a finite abelian group with decomposition $G=P_{1} \times$ $\cdots \times P_{k}$ into the product of its Sylow subgroups. Let $\omega \in Z^{3}\left(G, \mathbb{C}^{*}\right)$. Then there are cocycles $\eta_{i} \in Z^{3}\left(P_{i}, \mathbb{C}^{*}\right)$ such that $D^{\omega}(G)$ and $D^{\eta_{1}}\left(P_{1}\right) \otimes \cdots \otimes D^{\eta_{k}}\left(P_{k}\right)$ are equivalent as quasi-triangular quasi-bialgebras.

Because of this result, many of the questions we address regarding $D^{\omega}(G)(G$ abelian) can be reduced to the case that $G$ is a $p$-group for some prime $p$. 


\section{Eilenberg-Mac Lane COHOMOlOGy}

Let $G$ be a group. The bar complex of $G$, denoted by $A_{0}(G)$, is the chain complex of abelian group

$$
\stackrel{\partial}{\longrightarrow} C_{3} \stackrel{\partial}{\longrightarrow} C_{2} \stackrel{\partial}{\longrightarrow} C_{1} \longrightarrow \mathbb{Z}
$$

where $C_{n}$ is the free abelian group generated by all $n$-tuples $\left(x_{1}, \ldots, x_{n}\right)$ of elements $x_{i}$ of $G$ and $\partial$ is a $\mathbb{Z}$-linear map defined by

$$
\begin{aligned}
\partial\left(x_{1}, \ldots, x_{n}\right)= & \left(x_{2}, \ldots, x_{n}\right)+\sum_{i=1}^{n-1}(-1)^{i}\left(x_{1}, \ldots, x_{i-1}, x_{i} x_{i+1}, \ldots, x_{n}\right) \\
& +(-1)^{n}\left(x_{1}, \ldots, x_{n-1}\right) .
\end{aligned}
$$

We will call $\left(x_{1}, \ldots, x_{n}\right) \in C_{n}$ a $n$-dimensional cell of $A_{0}(G)$. For any abelian group $\Pi, \operatorname{Hom}\left(A_{0}(G), \Pi\right)$ is a cochain complex. We will denote by $C^{n}(G, \Pi), Z^{n}(G, \Pi)$, $B^{n}(G, \Pi)$ and $H^{n}(G, \Pi)$ the dimension $n$-cochains, cocycles, coboundaries and cohomology classes of $\operatorname{Hom}\left(A_{0}(G), \Pi\right)$ respectively.

For any two cells $\left(x_{1}, \ldots, x_{n}\right)$ and $\left(y_{1}, \ldots, y_{m}\right)$ in $A_{0}(G)$, we can define a "shuffle" of the $n$ letters $x_{1}, \ldots, x_{n}$ through the $m$ letters $y_{1}, \ldots, y_{m}$ to be the $n+m$-tuple in which the order of the $x$ 's and the order of $y$ 's are preserved. The sign of the shuffle is the sign of the permutation required to bring the shuffled letters back to the standard shuffle $\left(x_{1}, \ldots, x_{n}, y_{1}, \ldots, y_{m}\right)$. Then, we can define the "star" product of $\left(x_{1}, \ldots, x_{n}\right) *\left(y_{1}, \ldots, y_{m}\right)$ to be the signed sum of the shuffles of the letters $x$ through the letters $y$.

Let $G$ be an abelian group. In the paper [Mac52], the author described a complex $A_{1}(G)$ in which the cells are symbols $\sigma=\left[\alpha_{1}\left|\alpha_{2}\right| \cdots \mid \alpha_{p}\right]$, with each $\alpha_{i}$ a cell of $A_{0}(G)$. The dimension of $\sigma$ is $p-1$ plus the sum of the dimensions of the $\alpha_{i}$, and the boundary of $\sigma$ is

$$
\partial \sigma=\sum_{i=1}^{p}(-1)^{\epsilon_{i-1}}\left[\alpha_{1}|\cdots| \partial \alpha_{i}|\cdots| \alpha_{p}\right]+\sum_{i=1}^{p}(-1)^{\epsilon_{i}}\left[\alpha_{1}|\cdots| \alpha_{i} * \alpha_{i+1}|\cdots| \alpha_{p}\right],
$$

where $\epsilon_{i}=1+\operatorname{dim}\left[a_{1}|\cdots| \alpha_{i}\right]$. For any abelian $\Pi, \operatorname{Hom}\left(A_{1}(G), \Pi\right)$ is a cochain complex. We denote by $C_{a b}^{n}(G, \Pi), Z_{a b}^{n}(G, \Pi), B_{a b}^{n}(G, \Pi)$ and $H_{a b}^{n}(G, \Pi)$ the dimension $n$-cochains, cocycles, coboundaries and cohomology classes of $\operatorname{Hom}\left(A_{1}(G), \Pi\right)$ respectively. Until the end of this section, we will tacitly assume that $\Pi$ is a divisible abelian. The underlying product in $\Pi$ will be written additively.

Notice that $A_{0}(G)$ is a subcomplex of $A_{1}(G)$. We have the exact sequence of complexes

$$
0 \longrightarrow A_{0}(G) \longrightarrow A_{1}(G) \longrightarrow B(G) \longrightarrow 0
$$

with $B(G)$ the quotient complex $A_{1}(G) / A_{0}(G)$. Then

$$
0 \longrightarrow \operatorname{Hom}(B(G), \Pi) \longrightarrow \operatorname{Hom}\left(A_{1}(G), \Pi\right) \longrightarrow \operatorname{Hom}\left(A_{0}(G), \Pi\right) \longrightarrow 0 \text {. }
$$

is exact and we have the long exact sequence

$$
\begin{gathered}
\cdots \stackrel{\delta}{\longrightarrow} H^{*}(B(G), \Pi) \longrightarrow H_{a b}^{*}(G, \Pi) \longrightarrow H^{*}(G, \Pi) \\
\stackrel{\delta}{\longrightarrow} H^{*+1}(B(G), \Pi) \longrightarrow H_{a b}^{*+1}(G, \Pi) \longrightarrow \cdots
\end{gathered}
$$

where $H^{n}(B(G), \Pi)$ is the $n$-th cohomology group of the cochain complex $\operatorname{Hom}(B(G), \Pi)$. 
The low dimensional cells in $B(G)$ are given in the following table:

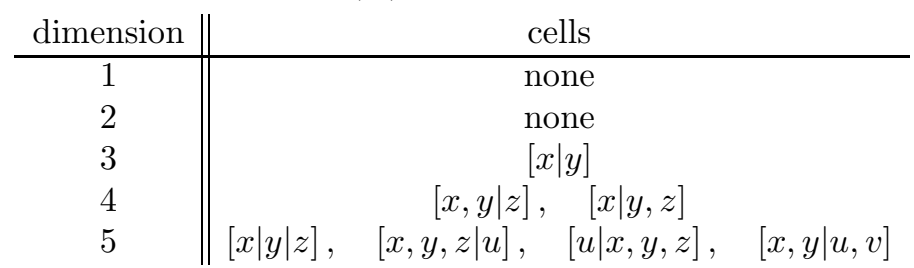

Since $H_{a b}^{2}(G, \Pi)=\operatorname{Ext}_{\mathbb{Z}}^{1}(G, \Pi)=0$, we have the exact sequence

$$
\begin{aligned}
0 \longrightarrow H^{2}(G, \Pi) & \stackrel{\delta}{\longrightarrow} H^{3}(B(G), \Pi) \longrightarrow H_{a b}^{3}(G, \Pi) \longrightarrow H^{3}(G, \Pi) \\
& \stackrel{\delta}{\longrightarrow} H^{4}(B(G), \Pi) .
\end{aligned}
$$

We are going to analyze the sequence (17) in some detail. Let us denote by $B_{n}(G)$ the group of $n$-chains of $B(G)$. For any cocycle $b: B^{3}(G) \longrightarrow \Pi$ and $x, y, z \in G$,

$$
\begin{aligned}
& b[\partial(x, y) \mid z]=0, \\
& b[x \mid \partial(y, z)]=0 .
\end{aligned}
$$

Hence, $b$ is a bicharacter of $G$. Conversely, it is also easy to see that any bicharacter on $G$ defines an element in $H^{3}(B(G), \Pi)$ uniquely. Hence, we have the following lemma.

Lemma 5.1. $H^{3}(B(G), \Pi)=\operatorname{Hom}(G \otimes G, \Pi)$.

Let $c \in Z^{2}(G, \Pi)$ and let $[c]$ be the corresponding cohomology class. There exists a natural isomorphism $\theta: H^{2}(G, \Pi) \longrightarrow \operatorname{Hom}\left(\bigwedge^{2} G, \Pi\right)$ defined by

$$
\theta([c])(x, y)=c(x, y)-c(y, x)
$$

for any $x, y \in G$ (cf. $[\mathrm{Bro} 82])$. On the other hand, the connecting homomorphism $\delta: H^{2}(G, \Pi) \longrightarrow H^{3}(B(G), \Pi)$ is given by

$$
([c])[x \mid y]=c((x) *(y))
$$

for $x, y \in G$. One can easily check that the following diagram commutes:

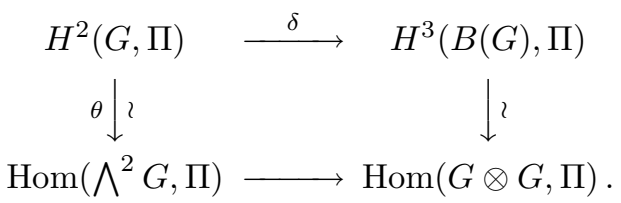

The bottom horizontal map in the diagram is the natural embedding. The exact sequence (17) is thus equivalent to the following exact sequence:

$$
\begin{aligned}
0 \longrightarrow \operatorname{Hom}\left(\bigwedge^{2} G, \Pi\right) & \longrightarrow \operatorname{Hom}(G \otimes G, \Pi) \longrightarrow H_{a b}^{3}(G, \Pi) \\
& \longrightarrow H^{3}(G, \Pi) \stackrel{\delta}{\longrightarrow} H^{4}(B(G), \Pi) .
\end{aligned}
$$

The 3-dimensional Eilenberg-Mac Lane cohomology group $H_{a b}^{3}(G, \Pi)$ was explicitly described in Mac52. The elements in $Z_{a b}^{3}(G, \Pi)$ are pairs $(f, d)$ where $f \in Z^{3}(G, \Pi)$ and $d \in C^{2}(G, \Pi)$ satisfying the following conditions:

$$
\begin{aligned}
& d(x y \mid z)-d(x \mid z)-d(y \mid z)+f(x, y, z)-f(x, z, y)+f(z, x, y)=0 \\
& d(x \mid y z)-d(x \mid y)-d(x \mid z)-f(x, y, z)+f(y, x, z)-f(y, z, x)=0 .
\end{aligned}
$$


The 3-dimensional cocycle $(f, d)$ is a coboundary if there exists $h \in C^{2}(G, \Pi)$ such that

$$
\begin{aligned}
f(x, y, z) & =\delta h(x, y, z), \\
d(x \mid y) & =h(x, y)-h(y, x) .
\end{aligned}
$$

Thus, the maps $\operatorname{Hom}(G \otimes G, \Pi) \longrightarrow H_{a b}^{3}(G, \Pi)$ and $H_{a b}^{3}(G, \Pi) \longrightarrow H^{3}(G, \Pi)$ in (21) are given by

$$
b \mapsto[(0, b)] \quad \text { and } \quad[(f, d)] \mapsto[f]
$$

respectively, where $[x]$ means the cohomology class associated to the cocycle $x$. To any $(f, d) \in Z_{a b}^{3}(G, \Pi)$, one can assign the function $t(x)=d(x \mid x)$, called its trace. Any trace is a quadratic function - a function $t: G \longrightarrow \Pi$ such that

(i) $t(a x)=a^{2} t(x)$ for $a \in \mathbb{Z}$, and

(ii) $b_{t}(x, y)=t(x+y)-t(x)-t(y)$ defines a bilinear function on $G$.

Theorem 5.2 (Eilenberg-Mac Lane). Let $Q(G, \Pi)$ consist of all quadratic functions from $G$ to $\Pi$. The function assigning to each cocycle its trace induces an isomorphism

$$
H_{a b}^{3}(G, \Pi) \stackrel{\cong}{\longrightarrow} Q(G, \Pi) .
$$

A map $F: B^{4}(G) \longrightarrow \Pi$ is a cocycle if it satisfies the following:

$$
\begin{aligned}
F[\partial(x, y, z) \mid u] & =0 \\
F[u \mid \partial(x, y, z)] & =0 \\
F[\partial(x, y) \mid u, v] & =F[x, y \mid \partial(u, v)] \\
F[x \mid(y) *(z)] & =-F[(x) *(y) \mid z] .
\end{aligned}
$$

$F$ is a coboundary if there is a function $d: B^{3}(G) \longrightarrow \Pi$ such that

$$
\begin{aligned}
d[\partial(x, y) \mid z] & =F[x, y \mid z], \\
d[x \mid \partial(y, z)] & =F[x \mid y, z] .
\end{aligned}
$$

Let $f \in Z^{3}(G, \Pi)$. The connecting homomorphism $\delta: H^{3}(G, \Pi) \longrightarrow H^{4}(B(G), \Pi)$ in (21) is given by $\delta[f]=[F]$ where $F: B^{4}(G) \longrightarrow \Pi$ is defined by

$$
\begin{aligned}
& F[x, y \mid z]=-f((x, y) *(z)), \\
& F[x \mid y, z]=f((x) *(y, z)) .
\end{aligned}
$$

Let $F: B^{4}(G) \longrightarrow \Pi$ be a cocycle. Define

$$
\bar{F}(x, y, z)=F[x, y \mid z]-F[y, x \mid z]
$$

for $x, y, z \in G$.

Lemma 5.3. The map

$$
\Psi:[F] \mapsto \bar{F}
$$

defines a group homomorphism from $H^{4}(B(G), \Pi)$ onto $\operatorname{Hom}\left(\bigwedge^{3} G, \Pi\right)$. The map splits if $G$ is finitely generated. 
Proof. Since $\partial(x, y)=\partial(y, x)$, one can check that $\Psi$ is well-defined. Let $F$ : $B^{4}(G) \longrightarrow \Pi$ be a cocycle. Then, for any $x, y, u, v \in G$,

$$
\begin{aligned}
\bar{F}(\partial(u, v), x, y) & =F[\partial(u, v), x \mid y]-F[x, \partial(u, v) \mid y] \\
& =-F[\partial(u, v) \mid x, y]+F[\partial(u, v) \mid y, x] \\
& =-F[u, v \mid \partial(x, y)]+F[u, v \mid \partial(y, x)] \\
& =0 .
\end{aligned}
$$

Therefore, $\bar{F}$ is linear at the first entry. Clearly,

$$
\bar{F}(x, y, z)=-\bar{F}(y, x, z) \quad \text { and } \quad \bar{F}(x, x, z)=0 .
$$

In particular, $\bar{F}$ is also linear at the second entry. Notice that

$$
\begin{aligned}
\bar{F}(x, y, z) & =F[x, y \mid z]-F[y, x \mid z] \\
& =F[x \mid z, y]-F[x \mid y, z] \\
& =-(F[x, z \mid y]-F[z, x \mid y]) \\
& =-\bar{F}(x, z, y) .
\end{aligned}
$$

Therefore, $\bar{F}$ is also linear at the third entry and hence $\bar{F} \in \operatorname{Hom}\left(\bigwedge^{3} G, \Pi\right)$.

Note that the map $\psi^{*}: H^{3}(G, \Pi) \longrightarrow \operatorname{Hom}\left(\bigwedge^{3} G, \Pi\right)$ given by

$$
\psi^{*}[\omega](x, y, z)=\sum_{\sigma} \operatorname{sgn}(\sigma) \omega(\sigma(x), \sigma(y), \sigma(z))
$$

with $\sigma$ running through the permutations of $x, y, z$ is a surjective homomorphism (cf. Bro82 ). It follows directly from (33) and (34) that the diagram

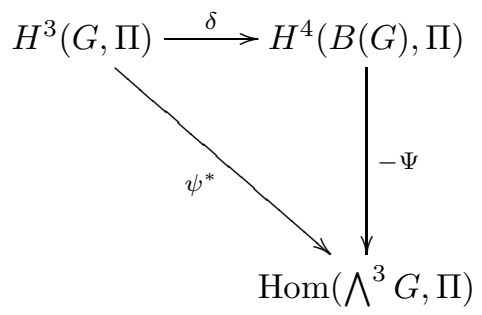

commutes. Hence, $\Psi$ is surjective. Moreover, if $G$ is finitely generated, the map $\psi^{*}$ has a linear section $s$. Then, $-\delta s$ is a linear section of $\Psi$.

For any $\beta \in Z_{a b}^{2}(G, \operatorname{Hom}(G, \Pi))$, define $F_{\beta}: B^{4}(G) \longrightarrow \Pi$ by

$$
F_{\beta}[x, y \mid z]=0 \quad \text { and } \quad F_{\beta}[x \mid y, z]=\beta(y, z)(x)
$$

for any $x, y, z \in G$. One can easily check that $F_{\beta} \in Z^{4}(B(G), \Pi)$. Moreover, $\Xi:[\beta] \longrightarrow\left[F_{\beta}\right]$ defines a linear map from $H_{a b}^{2}(G, \operatorname{Hom}(G, \Pi))$ to $H^{4}(B(G), \Pi)$.

Lemma 5.4. The sequence

$$
0 \longrightarrow H_{a b}^{2}(G, \operatorname{Hom}(G, \Pi)) \stackrel{\Xi}{\longrightarrow} H^{4}(B(G), \Pi) \stackrel{\Psi}{\longrightarrow} \operatorname{Hom}\left(\bigwedge^{3} G, \Pi\right) \longrightarrow 0
$$

is exact. In particular, if $G$ is finitely generated, the sequence is split exact.

Proof. If $\left[F_{\beta}\right]=0$, then there exists a function $d: B_{3}(G) \longrightarrow \Pi$ such that

$$
\begin{aligned}
0 & =d[\partial(x, y) \mid z], \\
\beta(y, z)(x) & =d[x \mid \partial(y, z)] .
\end{aligned}
$$


Hence, $d \in C^{1}(G, \operatorname{Hom}(G, \Pi))$ and $\beta=\delta d$. Therefore, $\Xi$ is injective.

Obviously, $\Psi \Xi[\beta]=0$ for $\beta \in Z_{a b}^{2}(G, \operatorname{Hom}(G, \Pi))$. Let $[F] \in \operatorname{ker} \Psi$. Then, we have

$$
F[x, y \mid z]=F[y, x \mid z]
$$

for any $x, y, z \in G$. Since $\Pi$ is divisible, there exists a map $\tau \in C^{2}(G, \Pi)$ such that

$$
\tau[\partial(x, y) \mid z]=F[x, y \mid z] .
$$

Then the map $F^{\prime}: B^{4}(G) \longrightarrow \Pi$ defined by

$$
F^{\prime}[x, y \mid z]=\tau[\partial(x, y) \mid z] \quad \text { and } \quad F^{\prime}[x \mid y, z]=\tau[x \mid \partial(y, z)]
$$

is a coboundary and hence $[F]=\left[F-F^{\prime}\right]$. Let $\beta(y, z)(x)=F[x \mid y, z]-F^{\prime}[x \mid y, z]$. Then,

$$
\begin{aligned}
\beta(y, z)(u)-\beta(y, z)(u v)+\beta(y, z)(v) & =F[\partial(u, v) \mid y, z]-\tau(\partial(u, v) \mid \partial(y, z)) \\
& =F[u, v \mid \partial(y, z)]-\tau(\partial(u, v) \mid \partial(y, z)) \\
& =\tau(\partial(u, v) \mid \partial(y, z))-\tau(\partial(u, v) \mid \partial(y, z)) \\
& =0 .
\end{aligned}
$$

Moreover, $\beta(\partial(x, y, z))(u)=F[u \mid \partial(x, y, z)]-F^{\prime}[u \mid \partial(x, y, z)]=0$. Therefore, $\beta \in$ $Z_{a b}^{2}(G, \operatorname{Hom}(G, \Pi))$ and $F_{\beta}=F-F^{\prime}$. Thus, $\Xi[\beta]=[F]$. The second statement follows directly from Lemma 5.3 .

\section{THE KERNEL OF $\Lambda$}

Let us return to the exact sequence (21) with $\Pi=\mathbb{C}^{*}$. We then have the exact sequence

$$
\begin{aligned}
0 \longrightarrow \operatorname{Hom}\left(\bigwedge^{2} G, \mathbb{C}^{*}\right) & \longrightarrow \operatorname{Hom}\left(G \otimes G, \mathbb{C}^{*}\right) \longrightarrow H_{a b}^{3}\left(G, \mathbb{C}^{*}\right) \\
& \longrightarrow H^{3}\left(G, \mathbb{C}^{*}\right) \stackrel{\delta}{\longrightarrow} H^{4}\left(B(G), \mathbb{C}^{*}\right) .
\end{aligned}
$$

Notice that for any $(f, d) \in Z_{a b}^{3}\left(G, \mathbb{C}^{*}\right), f \in Z^{3}\left(G, \mathbb{C}^{*}\right)$ and $f_{z}$ is a coboundary for $z \in G$ because

$$
f_{z}(x, y)=\frac{f(z, x, y) f(x, y, z)}{f(x, z, y)}=\frac{d(z \mid x) d(z \mid y)}{d(z \mid x y)} .
$$

Therefore, $[f] \in H^{3}\left(G, \mathbb{C}^{*}\right)_{a b}$ and hence the image of the map $H_{a b}^{3}\left(G, \mathbb{C}^{*}\right) \longrightarrow$ $H^{3}\left(G, \mathbb{C}^{*}\right)$ is contained in $H^{3}\left(G, \mathbb{C}^{*}\right)_{a b}$.

Let $f \in Z^{3}\left(G, \mathbb{C}^{*}\right)_{a b}$. Then $\delta[f]$ admits the representative $F \in Z^{4}\left(B(G), \mathbb{C}^{*}\right)$ defined by

$$
F[x, y \mid z]=f((x, y) *(z))^{-1} \quad \text { and } \quad F[x \mid y, z]=f((x) *(y, z)) .
$$

On the other hand, $\Xi \Lambda[f]$ has the representative $F^{\prime} \in Z^{4}\left(B(G), \mathbb{C}^{*}\right)$ given by

$$
F^{\prime}[x, y \mid z]=1 \quad \text { and } \quad F^{\prime}[x \mid y, z]=\frac{\tau_{y}(x) \tau_{z}(x)}{\tau_{y z}(x)} f_{x}(y, z)
$$

where $f_{x}(y, z)=\frac{\tau_{x}(y) \tau_{x}(z)}{\tau_{x}(y z)}$. Let

$$
d[x \mid y]=\tau_{y}(x) .
$$


Then $F^{\prime} / F=\delta d$. Hence $F^{\prime}$ and $F$ represent the same cohomology class in $H^{4}\left(B(G), \mathbb{C}^{*}\right)$ and the diagram

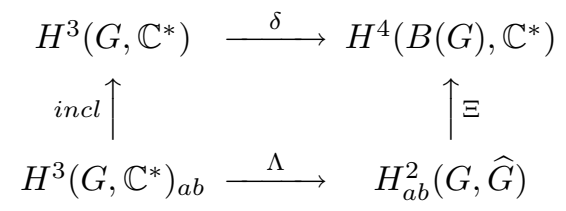

commutes. Moreover, we obtain an exact sequence

$$
\operatorname{Hom}\left(G \otimes G, \mathbb{C}^{*}\right) \longrightarrow H_{a b}^{3}\left(G, \mathbb{C}^{*}\right) \longrightarrow H^{3}\left(G, \mathbb{C}^{*}\right)_{a b} \stackrel{\Lambda}{\longrightarrow} H_{a b}^{2}(G, \widehat{G}) .
$$

Therefore,

$$
\operatorname{ker} \lambda=\operatorname{coker}\left(\operatorname{Hom}\left(G \otimes G, \mathbb{C}^{*}\right) \longrightarrow H_{a b}^{3}\left(G, \mathbb{C}^{*}\right)\right) .
$$

Since the map $\operatorname{Hom}\left(G \otimes G, \mathbb{C}^{*}\right) \longrightarrow H_{a b}^{3}\left(G, \mathbb{C}^{*}\right)$ is given by $b \mapsto[(1, b)]$,

$\operatorname{Im}\left(\operatorname{Hom}\left(G \otimes G, \mathbb{C}^{*}\right) \longrightarrow H_{a b}^{3}\left(G, \mathbb{C}^{*}\right)\right)=\left\{[(1, b)] \in H_{a b}^{3}\left(G, \mathbb{C}^{*}\right) \mid b\right.$ is a bicharacter $\}$.

Let $K\left(G, \mathbb{C}^{*}\right)$ be the group of quadratic forms which are the trace of some bicharacter. Then, by Theorem 5.2 we see that the following holds:

Proposition 6.1. For any abelian group $G$, $\operatorname{ker} \lambda=Q\left(G, \mathbb{C}^{*}\right) / K\left(G, \mathbb{C}^{*}\right)$.

Lemma 6.2. Let $G$ be a finite abelian group.

(i) Let $G=H \oplus L$ for subgroups $H, L$ of $G$. Then $t \in K\left(G, \mathbb{C}^{*}\right)$ if, and only if, $\left.t\right|_{H} \in K\left(H, \mathbb{C}^{*}\right)$ and $\left.t\right|_{L} \in K\left(L, \mathbb{C}^{*}\right)$.

(ii) Let $H$ be the Sylow 2-subgroup of $G$. Then

$$
Q\left(G, \mathbb{C}^{*}\right) / K\left(G, \mathbb{C}^{*}\right) \cong Q\left(H, \mathbb{C}^{*}\right) / K\left(H, \mathbb{C}^{*}\right)
$$

as abelian groups.

(iii) $Q\left(G, \mathbb{C}^{*}\right)^{2} \subseteq K\left(G, \mathbb{C}^{*}\right)$.

Proof. (i) The necessity of the statement is obvious. Let us assume $t \in K\left(G, \mathbb{C}^{*}\right)$ is such that $\left.t\right|_{H} \in K\left(H, \mathbb{C}^{*}\right)$ and $\left.t\right|_{L} \in K\left(L, \mathbb{C}^{*}\right)$. Let $b_{H}$ and $b_{L}$ be bicharacters of $H$ and $L$ respectively with

$$
\left.t\right|_{H}(x)=b_{H}(x, x) \text { and }\left.\quad t\right|_{L}(y)=b_{L}(y, y)
$$

for $x \in H$ and $y \in L$. Since $t \in Q\left(G, \mathbb{C}^{*}\right)$,

$$
c(u, v)=\frac{t(u v)}{t(u) t(v)}
$$

defines a bicharacter on $G$. Consider the map $b: G \times G \longrightarrow \mathbb{C}^{*}$ given by

$$
b\left(x_{1} y_{1}, x_{2} y_{2}\right)=b_{H}\left(x_{1}, x_{2}\right) c\left(x_{1}, y_{2}\right) b_{L}\left(y_{1}, y_{2}\right)
$$

for any $x_{1}, x_{2} \in H$ and $y_{1}, y_{2} \in L$. One can check directly that $b$ is a bicharacter on $G$. Moreover,

$$
b(x y, x y)=b_{H}(x, x) c(x, y) b_{L}(y, y)=t(x y) .
$$

Therefore, $t \in K\left(G, \mathbb{C}^{*}\right)$.

(ii) It suffices to show that $t \in K\left(G, \mathbb{C}^{*}\right)$ if, and only if, $\left.t\right|_{H} \in K\left(H, \mathbb{C}^{*}\right)$. Let $L$ be the subgroup of odd order elements of $G$. Then $G=H \oplus L$. Since $|L|$ is odd, $K\left(L, \mathbb{C}^{*}\right)=Q\left(L, \mathbb{C}^{*}\right)$. Hence, by (i), $t \in Q\left(G, \mathbb{C}^{*}\right)$ if, and only if, $\left.t\right|_{H} \in Q\left(H, \mathbb{C}^{*}\right)$. 
(iii) Since

$$
c(x, y)=\frac{t(x y)}{t(x) t(y)}
$$

is a bicharacter and $c(x, x)=t(x)^{2}$, the result follows.

Proposition 6.3. Let $G$ be a finite abelian group. Then $Q\left(G, \mathbb{C}^{*}\right) / K\left(G, \mathbb{C}^{*}\right) \cong$ $\Omega_{2}(G)$. In particular, $\operatorname{ker} \lambda_{G} \cong \Omega_{2}(G)$.

Proof. By virtue of Lemma 6.2(ii), we may assume that $G$ is a finite abelian 2group. Let us consider the case that $G$ is cyclic of order $2^{n}$ with the generator $y$. Then

$$
1=t(1)=t\left(y^{2^{n}}\right)=t(y)^{2^{2 n}}
$$

Since $y^{-1}=y^{2^{n}-1}$,

$$
t(y)=t\left(y^{2^{n}-1}\right)=t(y)^{2^{2 n}-2^{n+1}+1} .
$$

Hence, $t(y)$ is a $2^{n+1}$ th root of unity. Conversely, for any $2^{n+1}$ th root of unity $\xi, t\left(y^{r}\right)=\xi^{r^{2}}$ defines a quadratic function. Hence $Q\left(G, \mathbb{C}^{*}\right) \cong \mathbb{Z}_{2^{n+1}}$. Obviously, $K\left(G, \mathbb{C}^{*}\right)$ is a proper subgroup $Q\left(G, \mathbb{C}^{*}\right)$ and $Q\left(G, \mathbb{C}^{*}\right)^{2} \subseteq K\left(G, \mathbb{C}^{*}\right)$ by Lemma 6.2 (iii). Since $Q\left(G, \mathbb{C}^{*}\right)^{2}$ is the largest proper subgroup of $Q\left(G, \mathbb{C}^{*}\right), Q\left(G, \mathbb{C}^{*}\right)^{2}=$ $K\left(Q, \mathbb{C}^{*}\right)$. Hence, $Q\left(G, \mathbb{C}^{*}\right) / K\left(G, \mathbb{C}^{*}\right) \cong \mathbb{Z}_{2} \cong \Omega_{2}(G)$.

Now, we consider the general case. Let $G=C_{1} \oplus \cdots \oplus C_{l}$ where $C_{1}, \ldots, C_{l}$ are cyclic subgroups of $G$. Consider the map $p: Q\left(G, \mathbb{C}^{*}\right) \longrightarrow Q\left(C_{1}, \mathbb{C}^{*}\right) / K\left(C_{1}, \mathbb{C}^{*}\right) \times$ $\cdots \times Q\left(C_{l}, \mathbb{C}^{*}\right) / K\left(C_{l}, \mathbb{C}^{*}\right)$ defined by

$$
p(t)=\left(t_{1}, \ldots, t_{l}\right)
$$

where $t_{i}$ is the coset $\left.t\right|_{C_{i}} K\left(C_{i}, \mathbb{C}^{*}\right)$. Obviously, $p$ is an epimorphism. By Lemma $6.2(\mathrm{i}), \operatorname{ker} p=K\left(G, \mathbb{C}^{*}\right)$. Hence,

$$
\frac{Q\left(G, \mathbb{C}^{*}\right)}{K\left(G, \mathbb{C}^{*}\right)} \cong \frac{Q\left(C_{1}, \mathbb{C}^{*}\right)}{K\left(C_{1}, \mathbb{C}^{*}\right)} \times \cdots \times \frac{Q\left(C_{l}, \mathbb{C}^{*}\right)}{K\left(C_{l}, \mathbb{C}^{*}\right)} .
$$

As $\frac{Q\left(C_{i}, \mathbb{C}^{*}\right)}{K\left(C_{i}, \mathbb{C}^{*}\right)} \cong \mathbb{Z}_{2}, \frac{Q\left(G, \mathbb{C}^{*}\right)}{K\left(G, \mathbb{C}^{*}\right)} \cong \mathbb{Z}_{2}^{l} \cong \Omega_{2}(G)$.

Corollary 6.4. If $G$ is a finite abelian group of odd order, then $\Lambda$ is injective.

Corollary 6.5. Let $G$ be a finite abelian group and $H$ the Sylow 2-subgroup of $G$. Let $H=\bigoplus_{i=1}^{l} C_{i}$ be a cyclic subgroup decomposition of $H$. Let $p_{i}: H \longrightarrow C_{i}$ be the natural projection associated to the decomposition and let $p_{i}^{*}: H^{3}\left(C_{i}, \mathbb{C}^{*}\right) \longrightarrow$ $H^{3}\left(G, \mathbb{C}^{*}\right)$ be the associated inflation of $p_{i}$. Then

$$
\operatorname{ker} \lambda_{G}=\sum_{i=1}^{l} p_{i}^{*}\left(\operatorname{ker} \lambda_{C_{i}}\right) \text {. }
$$

Proof. Note that the exact sequence (39) is natural in $G$. Therefore, we have the commutative diagram

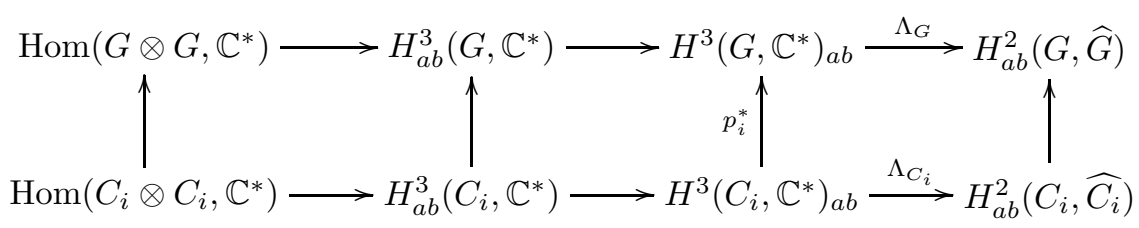


with exact rows. Therefore, $p_{i}^{*}\left(\operatorname{ker} \lambda_{C_{i}}\right) \subseteq \operatorname{ker} \Lambda_{G}$ for $i=1, \ldots, l$. Hence,

$$
\sum_{i=1}^{l} p_{i}^{*}\left(\operatorname{ker} \lambda_{C_{i}}\right) \subseteq \operatorname{ker} \lambda_{G}
$$

Since $p_{i}^{*}$ is injective and $\left\{\operatorname{Im} p_{i}^{*}\right\}$ is $\mathbb{Z}$-linearly independent in $H^{3}\left(G, \mathbb{C}^{*}\right)_{a b}$,

$$
\sum_{i=1}^{l} p_{i}^{*}\left(\operatorname{ker} \lambda_{C_{i}}\right) \cong \bigoplus_{i=1}^{l} \operatorname{ker} \Lambda_{C_{i}} \cong \mathbb{Z}_{2}^{l} .
$$

Therefore, the equality in the statement follows.

\section{The cohomology group $H^{3}\left(G, \mathbb{C}^{*}\right)_{a b}$}

Definition 7.1. Let $G$ be an abelian group and $M$ be a left $G$-module. For $g \in G$, the group homomorphism $m_{g}: C_{\bullet-1}(G) \longrightarrow C_{\bullet}(G)$ given by $y \mapsto y * g$ is a chain map. We denote by $D_{g, M}^{\bullet}: H^{\bullet}(G, M) \longrightarrow H^{\bullet-1}(G, M)$ the map induced by $m_{g}$ and define $D_{g, M}^{0}$ to be the trivial map. We will also denote $H^{\bullet}(G, M)_{a b}=$ $\bigcap_{g \in G} \operatorname{ker} D_{g, M}^{\bullet}$. When $M$ is the trivial module $\mathbb{C}^{*}, D_{g, M}$ will simply be written as $D_{g}$. In this case, one can easily see that $H^{3}(G, M)_{a b}$ coincides with $H^{3}\left(G, \mathbb{C}^{*}\right)_{a b}$ defined previously.

Lemma 7.2. For any positive integer $n \geq 1$,

$$
H^{n}\left(G, \mathbb{C}^{*}\right)_{a b} \cong H^{n+1}(G, \mathbb{Z})_{a b}
$$

and

$$
\frac{H^{n}\left(G, \mathbb{C}^{*}\right)}{H^{n}\left(G, \mathbb{C}^{*}\right)_{a b}} \cong \frac{H^{n+1}(G, \mathbb{Z})}{H^{n+1}(G, \mathbb{Z})_{a b}}
$$

Proof. Recall that, for $n \geq 1, H^{n}\left(G, \mathbb{C}^{*}\right) \stackrel{\delta}{\cong} H^{n+1}(G, \mathbb{Z})$ under the connecting maps of the long exact sequence associated to the exact sequence of trivial $G$-modules

$$
0 \longrightarrow \mathbb{Z} \longrightarrow \mathbb{C} \stackrel{\exp }{\longrightarrow} \mathbb{C}^{*} \longrightarrow 1 \text {. }
$$

Since the diagram

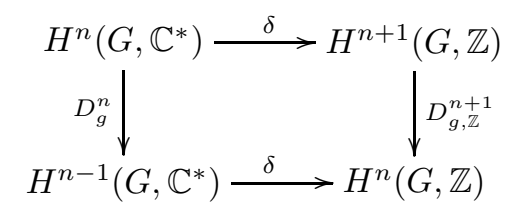

commutes for $g \in G$, the result follows.

Remark 7.3. Let $G$ be an abelian group. For any $g \in G$, the map $D_{g, \mathbb{Z}}=\bigoplus_{n \geq 0} D_{g, \mathbb{Z}}^{n}$ is a graded derivation on $\bigoplus_{n \geq 0} H^{n}(G, \mathbb{Z})$ with respect to the cup product. Hence, $\operatorname{ker} D_{g, \mathbb{Z}}$ is a graded subring of $H^{\bullet}(G, \mathbb{Z})$. Since

$$
H^{\bullet}(G, \mathbb{Z})_{a b}=\bigcap_{g \in G} \operatorname{ker} D_{g, \mathbb{Z}}
$$

$H^{\bullet}(G, \mathbb{Z})_{a b}$ is a graded subring of $H^{\bullet}(G, \mathbb{Z})$. In particular, if $G$ is a finite cyclic group, $H^{\bullet}(G, \mathbb{Z})$ is generated by $H^{2}(G, \mathbb{Z})$. As $H^{1}(G, \mathbb{Z})=0, H^{2}(G, \mathbb{Z})=H^{2}(G, \mathbb{Z})_{a b}$. Therefore, $H^{\bullet}(G, \mathbb{Z})_{a b}=H^{\bullet}(G, \mathbb{Z})$ in case $G$ is cyclic. 
Lemma 7.4. For any abelian group $G, H^{2}\left(G, \mathbb{C}^{*}\right)_{a b}$ is trivial and

$$
\frac{H^{3}\left(G, \mathbb{C}^{*}\right)}{H^{3}\left(G, \mathbb{C}^{*}\right)_{a b}} \cong \operatorname{Hom}\left(\bigwedge^{3} G, \mathbb{C}^{*}\right)
$$

Proof. For $[f] \in H^{2}\left(G, \mathbb{C}^{*}\right)_{a b}, f(x, y) / f(y, x)=1$. Hence, $f$ is a coboundary. Recall that the map $\varphi^{*}: H^{3}\left(G, \mathbb{C}^{*}\right) \longrightarrow \operatorname{Hom}\left(\bigwedge^{3} G, \mathbb{C}^{*}\right)$ defined by equation (36) is a split epimorphism. Moreover, for any normalized 3-cocycle $\omega$,

$$
\varphi *([\omega])(x, y, z)=\omega_{x}(y, z) / \omega_{x}(z, y)
$$

for any $x, y, z \in G$. Therefore, $\operatorname{ker} \varphi^{*}=H^{3}\left(G, \mathbb{C}^{*}\right)_{a b}$.

Let $G$ be a finite abelian group with a cyclic subgroup decomposition $G=$ $\bigoplus_{i=1}^{l} C_{i}$. Let $\left\{p_{i}\right\}$ be the projections of $G$ associated to the decomposition and $P_{i}^{\bullet}: H^{\bullet}\left(C_{i}, \mathbb{Z}\right) \longrightarrow H^{\bullet}(G, \mathbb{Z})$ the induced graded ring homomorphism with respect to cup product. Let $P_{C_{1}, \ldots, C_{l} ; G}^{\bullet} H^{\bullet}\left(C_{1}, \mathbb{Z}\right) \otimes \cdots \otimes H^{\bullet}\left(C_{l}, \mathbb{Z}\right) \longrightarrow H^{\bullet}(G, \mathbb{Z})$ be the graded ring monomorphism defined by

$$
P_{C_{1}, \ldots, C_{l} ; G}^{\bullet}\left(f_{1} \otimes \cdots \otimes f_{l}\right)=P_{1}\left(f_{1}\right) \cup \cdots \cup P_{l}\left(f_{l}\right) .
$$

Proposition 7.5. Let $G$ be a finite abelian group with a cyclic subgroup decomposition $G=\bigoplus_{i=1}^{l} C_{i}$. Then

$$
H^{4}(G, \mathbb{Z})_{a b}=\operatorname{Im} P_{C_{1}, \ldots, C_{l} ; G}^{4} \cdot
$$

In particular,

$$
H^{4}(G, \mathbb{Z})_{a b}=\bigoplus_{n_{1}+\cdots+n_{l}=4} H^{n_{1}}\left(C_{1}, \mathbb{Z}\right) \otimes \cdots \otimes H^{n_{l}}\left(C_{l}, \mathbb{Z}\right) .
$$

Proof. We proceed by induction on $l$. The case $l=1$ follows from Remark 7.3 Assume $l>1$ and $H=\bigoplus_{i=2}^{l} C_{i}$. Then $\operatorname{Im} P_{C_{1}, \ldots, C_{l} ; G}^{\bullet} \subseteq \operatorname{Im} P_{C_{1}, H ; G}^{\bullet}$. Moreover, by the Künneth theorem,

$$
\frac{H^{4}(G, \mathbb{Z})}{\operatorname{Im} P_{C_{1}, \ldots, C_{l} ; G}^{4}} \cong \frac{\operatorname{Im} P_{C_{1}, H ; G}^{4}}{\operatorname{Im} P_{C_{1}, \ldots, C_{l} ; G}^{4}} \oplus \operatorname{Tor}\left(H^{3}\left(C_{1}, \mathbb{Z}\right), H^{2}(H, \mathbb{Z})\right)
$$

Note that

$$
\frac{\operatorname{Im} P_{C_{1}, H ; G}^{4}}{\operatorname{Im} P_{C_{1}, \ldots, C_{l} ; G}^{4}} \cong \frac{H^{4}(G, \mathbb{Z})}{P_{C_{2}, \ldots, C_{l} ; H}^{4}} .
$$

By induction and Lemma 7.4 ,

$$
\frac{\operatorname{Im} P_{C_{1}, H ; G}^{4}}{\operatorname{Im} P_{C_{1}, \ldots, C_{l} ; G}^{4}} \cong \frac{H^{4}(G, \mathbb{Z})}{H^{4}(G, \mathbb{Z})_{a b}} \cong \bigwedge^{3} G
$$

Since

$$
\begin{gathered}
\operatorname{Tor}\left(H^{2}\left(C_{1}, \mathbb{Z}\right), H^{3}(H, \mathbb{Z})\right) \cong C_{2} \otimes H^{3}(H, \mathbb{Z}) \cong C_{2} \otimes H^{2}\left(H, \mathbb{C}^{*}\right) \cong C_{2} \otimes \bigwedge^{2} H \\
\frac{H^{4}(G, \mathbb{Z})}{\operatorname{Im} P_{C_{1}, \ldots, C_{l} ; G}^{4}} \cong\left(C_{2} \otimes \bigwedge^{2} H\right) \oplus \bigwedge^{3} H \cong \bigwedge^{3} G .
\end{gathered}
$$

Hence, by Lemma 7.4 and Lemma 7.2 ,

$$
\frac{H^{4}(G, \mathbb{Z})}{\operatorname{Im} P_{C_{1}, \ldots, C_{l} ; G}^{4}} \cong \frac{H^{4}(G, \mathbb{Z})}{H^{4}(G, \mathbb{Z})_{a b}}
$$


Thus, $\left|\operatorname{Im} P_{C_{1}, \ldots, C_{l} ; G}^{4}\right|=\left|H^{4}(G, \mathbb{Z})_{a b}\right|$. Notice that $\operatorname{Im} P_{C_{1}, \ldots, C_{l} ; G}^{\bullet}$ is the graded subring of $H^{\bullet}(G, \mathbb{Z})$ generated by $H^{2}(G, \mathbb{Z})$. Since $H^{2}(G, \mathbb{Z})=H^{2}(G, \mathbb{Z})_{a b}$, $\operatorname{Im} P_{C_{1}, \ldots, C_{l} ; G}^{\bullet} \subseteq H^{\bullet}(G, \mathbb{Z})_{a b}$.

\section{The Image of $\Lambda_{G}$}

Let $G$ be a finite abelian group and

$$
E: \quad 1 \longrightarrow \widehat{G} \longrightarrow \Gamma \longrightarrow G \longrightarrow 1
$$

an abelian central extension of $G$ by $\widehat{G}$. By applying the functor $\widehat{?}=\operatorname{Hom}\left(?, \mathbb{C}^{*}\right)$ to this exact sequence, we can obtain another abelian central extension of $G$ by $\widehat{G}$, namely,

$$
\epsilon(E): \quad 1 \longrightarrow \widehat{G} \longrightarrow \widehat{\Gamma} \longrightarrow G \longrightarrow 1
$$

where $G$ and $\widehat{\widehat{G}}$ are naturally identified. Obviously, if $E_{1}$ and $E_{2}$ are equivalent abelian central extensions of $G$ by $\widehat{G}$, then so are $\epsilon\left(E_{1}\right)$ and $\epsilon\left(E_{2}\right)$. Therefore, $\epsilon$ induces a map on $H_{a b}^{2}(G, \widehat{G})$. We will denote this map by the same symbol $\epsilon$.

Let $\beta$ be a normalized abelian cocycle in $Z_{a b}^{2}(G, \widehat{G})$. Then the set $\Gamma=\widehat{G} \times G$ equipped with the multiplication

$$
(\alpha, x) \cdot(\lambda, y)=(\alpha \lambda \beta(x, y), x y)
$$

is an abelian group. Moreover, a central extension of $G$ by $\widehat{G}$ associated to $[\beta]$ is given by

$$
1 \longrightarrow \widehat{G} \stackrel{i}{\longrightarrow} \Gamma \stackrel{p}{\longrightarrow} G \longrightarrow 1
$$

where $i(\alpha)=(\alpha, 1)$ and $p(\alpha, x)=x$ for $\alpha \in \widehat{G}$ and $x \in G$. Let $\tau_{x}$ be a normalized 1-cochain such that

$$
\delta \tau_{x}(g, h)=\beta(g, h)(x)
$$

for any $g, h \in G$. Let any $x \in G$, denoted by $\bar{x}$ be a fixed element in $\widehat{\Gamma}$ such that $\widehat{i}(\bar{x})=x$ and let $\overline{1}$ be the identity element of $\widehat{\Gamma}$. Then, there exist a normalized cocycle $\beta^{\prime} \in Z^{2}(G, \widehat{G})$ such that

$$
\overline{x y}=\widehat{p}\left(\beta^{\prime}(x, y)\right) \bar{x} \cdot \bar{y}
$$

for any $x, y \in G$. Obviously, $\epsilon[\beta]=\left[\beta^{\prime}\right]$.

Let $\tau_{x}^{\prime}(g)=\bar{x}(1, g)$ for any $g, x \in G$. Then

$$
\beta^{\prime}(x, y)(g)=\frac{\tau_{x}^{\prime}(g) \tau_{y}^{\prime}(g)}{\tau_{x y}^{\prime}(g)}
$$

for $x, y, g \in G$. Since $\bar{x} \in \widehat{\Gamma}$,

$$
\tau_{x}^{\prime}(g) \tau_{x}^{\prime}(h)=\tau_{x}^{\prime}(g h) \beta(g, h)(x) .
$$

Therefore, $\tau_{x}^{\prime}=\tau_{x} \lambda_{x}$ for some $\lambda_{x} \in \widehat{G}$. Define

$$
b_{1}^{\prime}(x, y)(g)=\frac{\tau_{x}(g) \tau_{y}(g)}{\tau_{x y}(g)} \text { and } \lambda(x)(g)=\lambda_{x}(g) .
$$

Then $\beta^{\prime}=\beta_{1}^{\prime} \delta \lambda$ and hence $\beta_{1}^{\prime}$ is a normalized 2-cocycle in $Z_{a b}^{2}(G, \widehat{G})$ and

$$
\epsilon([\beta])=\left[\beta_{1}^{\prime}\right]
$$

By the formulae (43) and (44), we obtain 
Proposition 8.1. The map $\epsilon: H_{a b}^{2}(G, \widehat{G}) \longrightarrow H_{a b}^{2}(G, \widehat{G})$ is a group homomorphism.

Let us identify $\widehat{G}$ and $H^{2}(G, \mathbb{Z})$ and consider the map $\Upsilon: \widehat{G} \otimes \widehat{G} \longrightarrow H_{a b}^{2}(G, \widehat{G})$, which assigns to $\alpha \otimes \lambda$ the cohomology class $[\beta]$ with

$$
\beta(x, y)(g)=\alpha(g)^{\tilde{\lambda}(x, y)}
$$

where $\tilde{\lambda}$ is a normalized 2-cocycle in $Z^{2}(G, \mathbb{Z})$ corresponding to $\lambda$. Since $G$ is finite, one can easily see that $\Upsilon$ is an isomorphism.

Lemma 8.2. Let $T: \widehat{G} \otimes \widehat{G} \longrightarrow \widehat{G} \otimes \widehat{G}$ be the transposition automorphism. Then,

$$
\Upsilon T=\epsilon \Upsilon \text {. }
$$

Proof. Let $\alpha_{1}, \alpha_{2} \in \widehat{G}$ and $\tilde{\alpha_{i}}=\delta f_{i}$ for some $f_{i}: G \longrightarrow \mathbb{C}$ such that $\operatorname{Im} \delta f_{i} \subseteq \mathbb{Z}$ and $f_{i}(1)=0$. Then $\Upsilon\left(\alpha_{1} \otimes \alpha_{2}\right)$ is the cohomology class represented by $\beta$ given by

$$
\beta(x, y)(g)=\alpha_{1}(g)^{f_{2}(x)+f_{2}(y)-f_{2}(x y)} .
$$

Let

$$
\tau_{g}(x)=\alpha_{1}(g)^{f_{2}(x)} \alpha_{2}(x)^{f_{1}(g)} .
$$

Then $\beta(x, y)(g)=\delta \tau_{g}(x, y)$. By formulae (43) and (44), $\epsilon[\beta]=\left[\beta^{\prime}\right]$ where $\beta^{\prime}$ is given by

$$
\beta^{\prime}(x, y)(g)=\frac{\tau_{x}(g) \tau_{y}(g)}{\tau_{x y}(g)}=\alpha_{2}(g)^{f_{1}(x)+f_{1}(y)-f_{1}(x y)} .
$$

Hence, $\left[\beta^{\prime}\right]=\Upsilon\left(\alpha_{2} \otimes \alpha_{1}\right)$.

Lemma 8.3. For any normalized $\beta \in Z_{a b}^{2}(G, \widehat{G}), \xi(\beta)(x, y, z)=\beta(x, y)(z)$ defines a 3-cocycle in $Z^{3}\left(G, \mathbb{C}^{*}\right)$ and $[\xi(\beta)] \in H^{3}\left(G, \mathbb{C}^{*}\right)_{a b}$. Moreover, $\Lambda_{G}[\xi(\beta)]=[\beta] \epsilon[\beta]$.

Proof. For any normalized $\beta \in Z_{a b}^{2}(G, \widehat{G})$, one can easily check that $\omega=\xi(\beta)$ satisfies the 3 -cocycle identity. For any $z \in G$, let $\tau_{z}: G \longrightarrow \mathbb{C}^{*}$ such that $\beta(x, y)(z)=\delta \tau_{z}(x, y)$. Then

$$
\omega_{z}(x, y)=\delta \tau_{z}(x, y)
$$

and so $[\omega] \in H^{2}\left(G, \mathbb{C}^{*}\right)_{a b}$. Moreover, $\Lambda_{G}[\omega]$ can be represented by the 2 -cocycle $\beta_{1}$ given by

$$
\beta_{1}(x, y)(z)=\frac{\tau_{z}(x) \tau_{z}(y)}{\tau_{z}(x y)} \frac{\tau_{x}(z) \tau_{y}(z)}{\tau_{x y}(z)}
$$

However, from the foregoing we have $\left[\beta_{1}\right]=[\beta] \epsilon[\beta]$.

Theorem 8.4. Let $G$ be a finite abelian group. Then

$$
\operatorname{Im} \Lambda_{G}=\operatorname{Im} S
$$

where $S: H^{2}(G, \widehat{G}) \longrightarrow H^{2}(G, \widehat{G})$ is defined by $S[\beta]=[\beta] \epsilon[\beta]$ for $[\beta] \in H_{a b}^{2}(G, \widehat{G})$.

Proof. It follows from Lemma 8.3 that $\operatorname{Im} \Lambda_{G} \subseteq \operatorname{Im} S$. By virtue of Lemma 8.2 $\operatorname{Im} S \cong \operatorname{Im}(i d+T)$. Let $G=C_{1} \oplus \cdots \oplus C_{l}$ be a cyclic subgroup decomposition of $G$. As $\widehat{G} \cong G$, one can easily see that

$$
\operatorname{Im}(i d+T) \cong\left(\bigoplus_{i<j} C_{i} \otimes C_{j}\right) \oplus\left(\bigoplus_{i=1}^{l} \frac{C_{i}}{\left(C_{i}\right)_{2}}\right) .
$$


By Proposition 7.5 and Proposition 6.3

$$
H^{3}\left(G, \mathbb{C}^{*}\right)_{a b} \cong\left(\bigoplus_{i<j} C_{i} \otimes C_{j}\right) \oplus\left(\bigoplus_{i=1}^{l} C_{i}\right) \quad \text { and } \quad \operatorname{ker} \Lambda_{G} \cong \Omega_{2}(G)
$$

Therefore, $\left|\operatorname{Im} \Lambda_{G}\right|=|\operatorname{Im} S|$ and hence $\operatorname{Im} \Lambda_{G}=\operatorname{Im} S$.

Theorem 8.5. Let $G$ be a finite abelian 2-group and $\omega$ a normalized 3-cocycle such that $[\omega] \in H^{2}\left(G, \mathbb{C}^{*}\right)_{a b}$. Then $\Gamma^{\omega}(G)$ is a direct sum of an even number of cyclic subgroups.

Proof. By Theorem 8.4, $\Lambda_{G}[\omega]=[\beta] \epsilon[\beta]$ for some normalized $\beta \in Z_{a b}^{2}(G, \widehat{G})$. For $z \in G$, let $\tau_{z}: G \longrightarrow \mathbb{C}^{*}$ such that

$$
\beta(x, y)(z)=\delta \tau_{z}(x, y) .
$$

Then, $\Lambda_{G}[\omega]$ contains the normalized 2-cocycle $\sigma$ given by

$$
\sigma(x, y)(z)=\frac{\tau_{z}(x) \tau_{z}(y)}{\tau_{z}(x y)} \frac{\tau_{x}(z) \tau_{y}(z)}{\tau_{x y}(z)} .
$$

Consider the central extension associated to $\sigma$

$$
1 \longrightarrow \widehat{G} \stackrel{i}{\longrightarrow} \Gamma \stackrel{p}{\longrightarrow} G \longrightarrow 1
$$

as defined in (41) where $\Gamma$ the group with the underlying set $\widehat{G} \times G$ endowed with the multiplication

$$
(\alpha, x) \cdot(\lambda, y)=(\alpha \lambda \sigma(x, y), x y) .
$$

To show that $\Gamma$ is a direct sum of an even number of cyclic subgroups, it is enough to show that $\Gamma^{\prime}=p^{-1}\left(\Omega_{2}(G)\right) / i\left(\widehat{G}^{2}\right)$ is a direct sum of an even number of cyclic subgroups. For this, it suffices to show that $\Gamma^{\prime}$ admits a nonsingular alternating bilinear form valued in $\mathbb{C}^{*}$ Wal63. Define $b: \Gamma^{\prime} \otimes \Gamma^{\prime} \longrightarrow \mathbb{C}^{*}$ by

$$
b\left(\left(\alpha \widehat{G}^{2}, x\right),\left(\lambda \widehat{G}^{2}, y\right)\right)=\frac{\alpha(y)}{\lambda(x)} \frac{\tau_{y}(x)}{\tau_{x}(y)}
$$

for $\alpha, \lambda \in \widehat{G}$ and $x, y \in \Omega_{2}(G)$. One can easily see that $b$ is well-defined. The linearity of $b$ follows from the fact that

$$
\beta(x, y)=\frac{\tau_{x} \tau_{y}}{\tau_{x y}}
$$

is a character for any $x, y \in G$. The routine verification that $b$ is also nondegenerate and alternating will be left to the reader.

Remark 8.6. We summarize some of our results.

(i) We have an exact sequence

$$
\begin{aligned}
1 \longrightarrow \operatorname{Hom}\left(\bigwedge^{2} G, \mathbb{C}^{*}\right) & \longrightarrow \operatorname{Hom}\left(G \otimes G, \mathbb{C}^{*}\right) \longrightarrow H_{a b}^{3}\left(G, \mathbb{C}^{*}\right) \\
& \longrightarrow H^{3}\left(G, \mathbb{C}^{*}\right)_{a b} \stackrel{\Lambda_{G}}{\longrightarrow} \operatorname{Im} S \longrightarrow 1
\end{aligned}
$$

where $S$ is as in Theorem 8.4 .

(ii) If $|G|$ is odd, then $\Lambda$ induces an isomorphism

$$
H^{3}\left(G, \mathbb{C}^{*}\right)_{a b} \underset{\cong}{\stackrel{\Lambda}{\cong}} H_{a b}^{2}(G, \widehat{G})^{+}
$$


where $H_{a b}^{2}(G, \widehat{G})^{+}$is the group of $\epsilon$-invariants. (In this case, $\Lambda$ is an injection (Cor. 6.4) and $\operatorname{Im} S=H_{a b}^{2}(G, \widehat{G})^{+}$as follows from Theorem 8.4 and the fact that $\epsilon$ is an involution on the group $H_{a b}^{2}(G, \widehat{G})$.)

\section{The monoidal Category $D^{\omega}(G)$-Mod}

Let $G$ be a finite abelian group and $\omega \in Z^{3}\left(G, \mathbb{C}^{*}\right)_{a b}$. Adopt the notation introduced in section 3, and pick $\tau \in T(\omega)$. Then,

$$
\Gamma^{\omega}=\{\sigma(\alpha, x) \mid \alpha \in \widehat{G}, x \in G\}
$$

where $\sigma(\alpha, x)$ is given by (16). By Proposition 2.2 for any $x \in G$ and $\alpha \in \widehat{G}$, the element

$$
\chi(\alpha, x)=\sum_{g \in G} \alpha(g) \tau_{x}(g) f_{g, x}
$$

is an algebra map from $D^{\omega}(G)$ to $\mathbb{C}$ where $\left\{f_{x, g}\right\}$ is the dual basis of $\{e(g) \otimes x\}$. Denote by $V(\alpha, x)$ the irreducible representation of $D^{\omega}(G)$ associated to the algebra map $\chi(\alpha, x)$. Then,

$$
S=\{V(\alpha, x) \mid \alpha \in \widehat{G}, x \in G\}
$$

is a complete set of irreducible representations of $D^{\omega}(G)$ up to isomorphism and $e(h) \otimes y$ acts on $V(\alpha, x)$ as scalar multiplication by $\alpha(y) \tau_{x}(y) \delta_{h, x}$. Hence, as reflected in equation (8), the associativity map

$$
\begin{gathered}
a_{V(\alpha, x), V(\lambda, y), V(\mu, z)}:(V(\alpha, x) \otimes V(\lambda, y)) \otimes V(\mu, z) \\
\longrightarrow V(\alpha, x) \otimes(V(\lambda, y) \otimes V(\mu, z))
\end{gathered}
$$

in $D^{\omega}(G)$-Mod is the scalar $\omega(x, y, z)^{-1}$. Notice that the group structure on $S$ induced by the tensor product of $D^{\omega}(G)$-Mod is isomorphic to $\Gamma^{\omega}$ with the isomorphism $\sigma(\alpha, x) \mapsto V(\alpha, x)$. Hence, by the results of [TY98], we have the following lemma.

Lemma 9.1. Let $G$ be a finite abelian group and $\omega$ be a normalized 3-cocycle such that $[\omega] \in H^{3}\left(G, \mathbb{C}^{*}\right)_{a b}$. Then, $D^{\omega}(G)$-Mod is monoidally equivalent to $D^{\omega}(G)_{0}$-Mod if, and only if, infl $[\omega]$ is trivial, where infl : $H^{3}\left(G, \mathbb{C}^{*}\right) \longrightarrow$ $H^{3}\left(\Gamma^{\omega}, \mathbb{C}^{*}\right)$ is inflation along the projection $\Gamma^{\omega} \longrightarrow G$.

In fact, we have

Lemma 9.2. Suppose that $G$ is finite and abelian and that infl $[\omega]$ is trivial. Then $D^{\omega}(G)$ and $D^{\omega}(G)_{0}$ are gauge equivalent as quasi-bialgebras.

Proof. Since infl $[\omega]$ is trivial, there is a normalized 3-cochain $f$ on $\Gamma^{\omega}$ such that

$$
\operatorname{infl}(\omega)=\delta f .
$$

Let $\tau \in T(\omega)$ and let $E_{\sigma(\alpha, x)}=\frac{1}{|G|} e(x) \otimes \sum_{g \in G} \frac{g}{\alpha(g) \tau_{x}(g)}$. One can easily see that $E_{u} E_{v}=\delta_{u, v} E_{v}$ and $\Delta E_{u}=\sum_{s, t \in \Gamma^{\omega}} E_{s} \otimes E_{t}$. Set

$$
F=\sum_{u, v \in \Gamma^{\omega}} f(u, v)^{-1} E_{u} \otimes E_{v} .
$$

One can easily check that $F$ is invertible with

$$
F^{-1}=\sum_{u, v \in \Gamma^{\omega}} f(u, v) E_{u} \otimes E_{v} .
$$


As $f$ is normalized,

$$
(\varepsilon \otimes i d) F=1_{D}^{\omega}(G)=(i d \otimes \varepsilon)(F) .
$$

Moreover, by equation (47),

$$
\Phi=F_{23}(i d \otimes \Delta)(F) \Phi_{0}(\Delta \otimes i d)\left(F^{-1}\right) F_{12}^{-1}
$$

where $\Phi_{0}=1_{D}^{\omega}(G) \otimes 1_{D}^{\omega}(G) \otimes 1_{D}^{\omega}(G)$. Since $D^{\omega}(G)_{0}$ is a commutative, $D^{\omega}(G)$ is a twist of $D^{\omega}(G)_{0}$ by $F$, that is, $D^{\omega}(G)$ and $D^{\omega}(G)_{0}$ are gauge equivalent.

Lemma 9.3. infl $[\omega] \in \operatorname{ker} \Lambda_{\Gamma \omega}$.

Proof. Set $\bar{\omega}=$ infl $\omega$. Define $d: \Gamma^{\omega} \times \Gamma^{\omega} \longrightarrow \mathbb{C}^{*}$ by

$$
d[\sigma(\alpha, x) \mid \sigma(\lambda, y)]=\frac{1}{\alpha(y) \tau_{x}(y)} .
$$

One can check directly that $(\bar{\omega}, d) \in Z_{a b}^{3}\left(\Gamma^{\omega}, \mathbb{C}^{*}\right)$. By the exact sequence (39), we have the commutative diagram

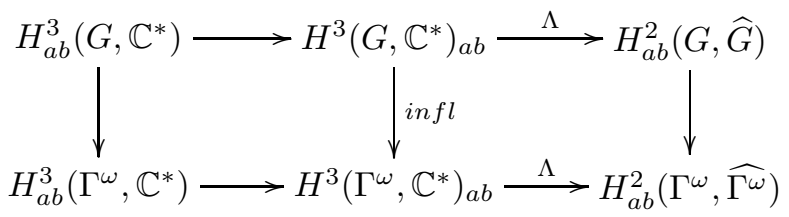

with the rows exact, where the vertical homomorphisms are induced by the epimorphism $\Gamma^{\omega} \longrightarrow G$. As

$$
[(\bar{\omega}, d)] \mapsto[\bar{\omega}]=\operatorname{infl}[\omega]
$$

it follows from the diagram that infl $[\omega] \in \operatorname{ker} \lambda_{\Gamma \omega}$.

Theorem 9.4. Let $G$ be a finite abelian group of odd order and $\omega_{1}, \omega_{2}$ normalized 3cocycles such that $\left[\omega_{1}\right],\left[\omega_{2}\right] \in H^{3}\left(G, \mathbb{C}^{*}\right)_{a b}$. Then, $D^{\omega_{1}}(G)$-Mod and $D^{\omega_{2}}(G)$-Mod are monoidally equivalent if, and only if, $\Gamma^{\omega_{1}} \cong \Gamma^{\omega_{2}}$ as groups.

Proof. Since the group structure, induced by the underlying tensor product, on the isomorphism classes of irreducible representations of $D^{\omega_{i}}(G)$ is isomorphic to $\Gamma^{\omega_{i}}$, $\Gamma^{\omega_{1}} \cong \Gamma^{\omega_{2}}$ as groups if $D^{\omega_{1}}(G)$-Mod and $D^{\omega_{2}}(G)$-Mod are monoidal equivalent. Conversely, assume that $\Gamma^{\omega_{1}} \cong \Gamma^{\omega_{2}}$ as groups. Then, $\mathbb{C} \Gamma^{\omega_{1}}$ and $\mathbb{C} \Gamma^{\omega_{2}}$ are isomorphic as Hopf algebras. By Lemma 9.3, infl $\left[\omega_{i}\right] \in \operatorname{ker} \Lambda_{\Gamma^{\omega_{i}}}$. As $G$ is of odd order, $\left|\Gamma^{\omega_{i}}\right|$ is odd, and it follows from Corollary 6.4 that $\operatorname{ker} \Lambda_{\Gamma^{\omega_{i}}}$ is trivial. Hence, by Lemma 9.2, $D^{\omega_{i}}(G)$ is equivalent to $\mathbb{C} \Gamma^{\omega_{i}}$ and hence $D^{\omega_{i}}(G)$-Mod is monoidally equivalent to the tensor category $\mathbb{C} \Gamma^{\omega_{i}}$-Mod with the usual associativity constraint.

Example 9.5. Theorem 9.4 is not true for groups of even order. For instance, take $G=\mathbb{Z}_{2}$. By Proposition 6.3 $\operatorname{ker} \Lambda_{G} \cong \mathbb{Z}_{2}$ and hence $\operatorname{ker} \Lambda_{G}=H^{3}\left(G, \mathbb{C}^{*}\right)$. Let $\omega_{1}$ be a normalized 3-cocycle on $G$ whose cohomology class is nontrivial and let $\omega_{0}=1$. As $\Lambda_{G}\left[\omega_{i}\right]$ is trivial $(i=0,1), \Gamma^{\omega_{i}} \cong \mathbb{Z}_{2} \times \mathbb{Z}_{2}$ as groups. Therefore, inflation of cohomology along $\Gamma^{\omega_{i}} \longrightarrow G$ is injective for $i=0,1$. Thus, infl $\left[\omega_{1}\right]$ is non-trivial, while infl $\left[\omega_{0}\right]$ is obviously trivial. Thanks to lemma 0.1 if $\mathbb{C}\left[\mathbb{Z}_{2} \times \mathbb{Z}_{2}\right]$-Mod is equipped with the usual associativity constraint, then $D^{\omega_{0}}(G)$-Mod is monoidally equivalent to $\mathbb{C}\left[\mathbb{Z}_{2} \times \mathbb{Z}_{2}\right]$-Mod but $D^{\omega_{1}}(G)$-Mod is not. Note that $\mathbb{C}\left[\mathbb{Z}_{4}\right]$ and $\mathbb{C}\left[\mathbb{Z}_{2} \times \mathbb{Z}_{2}\right]$ are the only 4-dimensional semisimple Hopf algebras up to isomorphism. As the fusion rule of $D^{\omega_{1}}(G)$-Mod is isomorphic to $\mathbb{Z}_{2} \times \mathbb{Z}_{2}, D^{\omega_{1}}(G)$-Mod and 
$\mathbb{C}\left[\mathbb{Z}_{4}\right]$-Mod are not monoidally equivalent. Hence, $D^{\omega_{1}}(G)$ cannot be obtained from any Hopf algebra by a twist.

Remark 9.6. In the paper DPR92, p. 92, the authors asked whether $D^{\omega}(G)$ can be obtained by twisting a Hopf algebra. Theorem 9.4 gives an affirmative answer to the question under the conditions stated in the theorem. However, in general, Example 9.5 gives a negative answer to the question.

\section{Gauge equivalence And Quadratic Forms}

Let $G$ be a finite abelian group, $\omega \in Z^{3}\left(G, \mathbb{C}^{*}\right)_{a b}$ and $\tau \in T(\omega)$. Then, as shown in the proof of Lemma 9.3 (infl $\left.\omega^{-1}, d_{\omega}\right) \in Z_{a b}^{3}\left(\Gamma^{\omega}(G), \mathbb{C}^{*}\right)$ where $d_{\omega}$ : $\Gamma^{\omega}(G) \times \Gamma^{\omega}(G) \longrightarrow \mathbb{C}^{*}$ is defined by

$$
d_{\omega}[\sigma(\alpha, x) \mid \sigma(\lambda, y)]=\alpha(y) \tau_{x}(y) .
$$

Define $q_{\omega}: \Gamma^{\omega}(G) \longrightarrow \mathbb{C}^{*}$ to be the trace of the abelian 3-cocycle $\left(\right.$ infl $\left.\omega^{-1}, d_{\omega}\right)$. Obviously, $q_{\omega}$ is a quadratic map given by

$$
q_{\omega}(\sigma(\alpha, x))=\alpha(x) \tau_{x}(x) .
$$

Denote by $b_{\omega}$ the symmetric bicharacter on $\Gamma^{\omega}(G)$ associated to $q_{\omega}$, namely, $b_{\omega}(x, y)=\frac{q_{\omega}(x y)}{q_{\omega}(x) q_{\omega}(y)}$. One can easily show that $b_{\omega}$ is given by

$$
b_{\omega}(\sigma(\alpha, x), \sigma(\lambda, y))=\alpha(y) \lambda(x) \tau_{x}(y) \tau_{y}(x)
$$

for any $\sigma(\alpha, x), \sigma(\lambda, y) \in \Gamma^{\omega}(G)$. Obviously, $b_{\omega}$ is non-degenerate on $\Gamma^{\omega}(G)$. Let $\langle\bullet, \bullet\rangle_{\omega}$ be the $\mathbb{C}$-linear extension of $b_{\omega}$ on $D^{\omega}(G)$.

Lemma 10.1. With the previous notation, the map $\varphi_{\omega}: D^{\omega}(G)_{0} \longrightarrow D^{\omega}(G)_{0}^{*}$, $\varphi_{\omega}: x \mapsto\langle x, \bullet\rangle_{\omega}$ is identical to the Hopf algebra isomorphism $\varphi: D^{\omega}(G)_{0} \longrightarrow$ $D^{\omega}(G)_{0}^{*}$ defined in (13). In particular, $\langle\bullet, \bullet\rangle_{\omega}$ is a non-degenerate symmetric bilinear form on $D^{\omega}(G)$. Moreover, for any $u, v \in \Gamma^{\omega}$,

$$
\left(\varphi_{\omega}(u) \otimes \varphi_{\omega}(v)\right) R=d_{\omega}[v \mid u]
$$

and

$$
q_{\omega}(u)=\left(\varphi_{\omega}(u) \otimes \varphi_{\omega}(u)\right) R
$$

where $R$ is the $\mathcal{R}$-matrix of $D^{\omega}(G)$.

Proof. To show that $\varphi_{\omega}=\varphi$, it suffices to show that $\varphi_{\omega}(u)=\varphi(u)$ for $u \in \Gamma^{\omega}$. Hence, it is enough to show that

$$
\varphi(u)(v)=\varphi_{\omega}(u)(v)=b_{\omega}(u, v)
$$

for any $u, v \in \Gamma^{\omega}$. However, it follows from (13) that

$$
\varphi(\sigma(\alpha, x))(\sigma(\lambda, y))=\alpha(y) \lambda(x) \tau_{x}(y) \tau_{y}(x)=b_{\omega}(\sigma(\alpha, x), \sigma(\lambda, y)) .
$$

Now, $\varphi_{\omega}(\sigma(\alpha, x))(e(g) \otimes y)=\alpha(y) \tau_{x}(y) \delta_{g, x}$. Thus,

$$
\left(\varphi_{\omega}(\sigma(\alpha, x)) \otimes \varphi_{\omega}(\sigma(\lambda, y))\right) R=\lambda(x) t_{y}(x)=d_{\omega}[\sigma(\lambda, y) \mid \sigma(\alpha, x)]
$$

and hence

$$
\left(\varphi_{\omega}(u) \otimes \varphi_{\omega}(u)\right) R=d_{\omega}[u \mid u]=q_{\omega}(u)
$$

for $u \in \Gamma^{\omega}$. 
Lemma 10.2. Let $\omega_{1}$ and $\omega_{2}$ be normalized 3-cocycles in $Z^{3}\left(G_{1}, \mathbb{C}^{*}\right)_{a b}$ and $Z^{3}\left(G_{2}, \mathbb{C}^{*}\right)_{a b}$ respectively. If $j: D^{\omega_{1}}\left(G_{1}\right)_{0} \longrightarrow D^{\omega_{2}}\left(G_{2}\right)_{0}$ is a bialgebra isomorphism, then $j^{t}: D^{\omega_{2}}\left(G_{2}\right)_{0} \longrightarrow D^{\omega_{1}}\left(G_{1}\right)_{0}$, defined by

$$
\left\langle j^{t}(u), v\right\rangle_{\omega_{1}}=\langle u, j(v)\rangle_{\omega_{2}}
$$

for any $u \in D^{\omega_{2}\left(G_{2}\right)}$ and $v \in D^{\omega_{1}\left(G_{1}\right)}$, is also a bialgebra isomorphism.

Proof. Consider the map $j^{\prime}: D^{\omega_{2}}\left(G_{2}\right)_{0} \longrightarrow D^{\omega_{1}}\left(G_{1}\right)_{0}$ given by

$$
j^{\prime}=\varphi_{\omega_{1}}^{-1} j^{*} \varphi_{\omega_{2}} \text {. }
$$

Since $j: D^{\omega_{1}}\left(G_{1}\right)_{0} \longrightarrow D^{\omega_{2}}\left(G_{2}\right)_{0}$ is a bialgebra isomorphism, then so is $j^{*}$ : $D^{\omega_{2}}\left(G_{2}\right)_{0}^{*} \longrightarrow D^{\omega_{1}}\left(G_{1}\right)_{0}^{*}$. It follows from Lemma 10.1 that $j^{\prime}$ is also a bialgebra isomorphism. Moreover, for any $u \in D^{\omega_{2}}\left(G_{2}\right)$ and $v \in D^{\omega_{1}}\left(G_{1}\right)$,

$$
\left\langle j^{\prime}(u), v\right\rangle_{\omega_{1}}=\left(\varphi_{\omega_{1}} j^{\prime}(u)\right)(v)=\left(j^{*} \varphi_{\omega_{2}}(u)\right)(v)=\varphi_{\omega_{2}}(j(u))(v)=\langle u, j(v)\rangle_{\omega_{2}} .
$$

Hence, $j^{\prime}=j^{t}$.

Definition 10.3. Let $\Gamma_{1}, \Gamma_{2}$ be abelian groups, and $b_{1}, b_{2}$ bicharacters on $\Gamma_{1}$ and $\Gamma_{2}$ respectively. The pairs $\left(\Gamma_{1}, b_{1}\right)$ and $\left(\Gamma_{2}, b_{2}\right)$ are said to be equivalent if there is a group isomorphism $j: \Gamma_{1} \longrightarrow \Gamma_{2}$ such that

$$
b_{2}(j(x), j(y))=b_{1}(x, y)
$$

for $x, y \in \Gamma_{1}$. Similarly, let $q_{1}: \Gamma_{1} \longrightarrow \mathbb{C}^{*}$ and $q_{2}: \Gamma_{2} \longrightarrow \mathbb{C}^{*}$ be quadratic maps. The pairs $\left(\Gamma_{1}, q_{1}\right)$ and $\left(\Gamma_{2}, q_{2}\right)$, which we call quadratic spaces, are said to be equivalent if there is a group isomorphism $j: \Gamma_{1} \longrightarrow \Gamma_{2}$ such that

$$
q_{2} j=q_{1} .
$$

The orthogonal sum of two quadratic spaces $\left(\Gamma_{1}, q_{1}\right)$ and $\left(\Gamma_{2}, q_{2}\right)$ is the quadratic space $\left(\Gamma_{1} \times \Gamma_{2}, q\right)$ where $q(x, y)=q_{1}(x) q_{2}(y)$.

Theorem 10.4. Let $G_{1}, G_{2}$ be finite abelian groups and $\omega_{1}, \omega_{2}$ normalized 3cocycles in $Z^{3}\left(G_{1}, \mathbb{C}^{*}\right)_{a b}$ and $Z^{3}\left(G_{2}, \mathbb{C}^{*}\right)_{a b}$, respectively. Then, $D^{\omega_{1}}\left(G_{1}\right)$ and $D^{\omega_{2}}\left(G_{2}\right)$ are gauge equivalent if, and only if, $\left(\Gamma^{\omega_{1}}, q_{\omega_{1}}\right)$ and $\left(\Gamma^{\omega_{2}}, q_{\omega_{2}}\right)$ are equivalent.

Proof. Suppose that $D^{\omega_{1}}\left(G_{1}\right)$ and $D^{\omega_{2}}\left(G_{2}\right)$ are equivalent as quasi-triangular quasiHopf algebras. Then there exists a gauge transform $F \in D^{\omega_{2}}\left(G_{2}\right) \otimes D^{\omega_{2}}\left(G_{2}\right)$ such that $D^{\omega_{1}}\left(G_{1}\right)$ and $D^{\omega_{2}}\left(G_{2}\right)_{F}$ are isomorphic as quasi-triangular quasibialgebras. Let $j: D^{\omega_{1}}\left(G_{1}\right) \longrightarrow D^{\omega_{2}}\left(G_{2}\right)_{F}$ be such an isomorphism. Then,

$$
(j \otimes j) R_{1}=F_{21} R_{2} F^{-1}
$$

where $R_{1}$ and $R_{2}$ are the $\mathcal{R}$-matrices of $D^{\omega_{1}}\left(G_{1}\right)$ and $D^{\omega_{2}}\left(G_{2}\right)$ respectively. Since $\Delta_{2}=\left(\Delta_{2}\right)_{F}, D^{\omega_{2}}\left(G_{2}\right)_{F}$ and $D^{\omega_{2}}\left(G_{2}\right)_{0}$ are identical as bialgebras. By Lemma 10.2 $j^{t}: D^{\omega_{2}}\left(G_{2}\right)_{0} \longrightarrow D^{\omega_{1}}\left(G_{1}\right)_{0}$ is a bialgebra isomorphism. In particular, $j^{t}\left(\Gamma^{\omega_{2}}\right)=$ $\Gamma^{\omega_{1}}$. Let $\left\{E_{u}\right\}_{u \in \Gamma \omega_{2}}$ be the dual basis of $\Gamma^{\omega_{2}}$ with respect to the pairing $\langle\bullet, \bullet\rangle_{\omega_{2}}$. Let $F=\sum_{u, v \in \Gamma^{\omega_{2}}} f(u, v) E_{u} \otimes E_{v}$. For any $u \in \Gamma^{\omega_{2}}$, apply $\varphi_{\omega_{2}}(u) \otimes \varphi_{\omega_{2}}(u)$ to equation (51). The left-hand side of the equation becomes

$$
\left(\varphi_{\omega_{2}}(u) \otimes \varphi_{\omega_{2}}(u)\right)(j \otimes j) R_{1}=\left(\varphi_{\omega_{1}}\left(j^{t} u\right) \otimes \varphi_{\omega_{1}}\left(j^{t} u\right)\right) R_{1}=q_{\omega_{1}}\left(j^{t}(u)\right)
$$

and the right-hand side becomes

$$
\left(\varphi_{\omega_{2}}(u) \otimes \varphi_{\omega_{2}}(u)\right)\left(F_{21} R_{2} F^{-1}\right)=q_{\omega_{2}}(u) f(u, u) f(u, u)^{-1}=q_{\omega_{2}}(u) .
$$


Therefore, $\left(\Gamma^{\omega_{1}}, q_{\omega_{1}}\right)$ and $\left(\Gamma^{\omega_{2}}, q_{\omega_{2}}\right)$ are equivalent. Conversely, assume that there exists an isomorphism $j: \Gamma^{\omega_{2}} \longrightarrow \Gamma^{\omega_{1}}$ such that $q_{\omega_{1}} j=q_{\omega_{2}}$. Set

$$
\hat{j}\left(i n f l \omega_{1}\right)(u, v, w)=\operatorname{infl} \omega_{1}(j(u), j(v), j(w))
$$

and

$$
\hat{j} d_{\omega_{1}}[u \mid v]=d_{\omega_{1}}[j(u) \mid j(v)]
$$

for any $u, v \in \Gamma^{\omega_{2}}$. Then, $\left(\hat{j}\left(i n f l \omega_{1}^{-1}\right), \hat{j}\left(d_{\omega_{1}}\right)\right)$ is also an abelian 3-cocycle of $\Gamma^{\omega_{2}}$. The trace of this cocycle is $q_{\omega_{1}} j$. By Theorem [5.2, there exists a normalized 2-cochain $f$ on $\Gamma^{\omega_{2}}$ such that

$$
\begin{aligned}
\operatorname{infl} \omega_{1}^{-1}(j(u), j(v), j(w)) & =\omega_{2}^{-1}(u, v, w) \delta f(u, v, w), \\
d_{\omega_{1}}[j(u) \mid j(v)] & =d_{\omega_{2}}[u \mid v] f(u, v) / f(v, u)
\end{aligned}
$$

for any $u, v, w \in \Gamma^{\omega_{2}}$. Let

$$
F=\sum_{u, v \in \Gamma^{\omega_{2}}} f(u, v) E_{u} \otimes E_{v} .
$$

Notice that $\epsilon\left(E_{u}\right)=\left\langle E_{u}, \sigma(1,1)\right\rangle=\delta_{1, u}$ and $E_{u} E_{v}=\delta_{u, v} E_{u}$ for any $u, v \in \Gamma^{\omega_{2}}$. This implies that $F$ is invertible in $D^{\omega_{2}}\left(G_{2}\right) \otimes D^{\omega_{2}}\left(G_{2}\right)$ and

$$
(i d \otimes \epsilon) F=1_{D}^{\omega_{2}\left(G_{2}\right)}=(\epsilon \otimes i d) F .
$$

For simplicity, we denote the linear extension of $j$ from $D^{\omega_{2}}\left(G_{2}\right)_{0}$ to $D^{\omega_{1}}\left(G_{1}\right)_{0}$ by the same symbol $j$. Obviously, $j$ is a bialgebra isomorphism. By Lemma 10.2 $j^{t}$ is also a bialgebra isomorphism. For any $u, v, w \in \Gamma^{\omega_{2}}$,

$$
\begin{aligned}
& \left(\varphi_{\omega_{2}}(u) \otimes \varphi_{\omega_{2}}(v) \otimes \varphi_{\omega_{2}}(w)\right)\left(j^{t} \otimes j^{t} \otimes j^{t}\right) \Phi_{1} \\
& \quad=\left(\varphi_{\omega_{1}}(j(u)) \otimes \varphi_{\omega_{1}}(j(v)) \otimes \varphi_{\omega_{1}}(j(w))\right) \Phi_{1} \\
& \quad=i n f l \omega_{1}^{-1}(j(u), j(v), j(w))
\end{aligned}
$$

and

$$
\begin{aligned}
\left(\varphi_{\omega_{2}}(u) \otimes \varphi_{\omega_{2}}(v) \otimes \varphi_{\omega_{2}}(w)\right)\left(F_{23}(i d \otimes \Delta)(F) \Phi_{2}(\Delta \otimes i d)\left(F^{-1}\right) F_{12}^{-1}\right) \\
=\left(i n f l \omega_{2}\right) \delta f(u, v, w) .
\end{aligned}
$$

Hence, by equation (52), we obtain

$$
\left(j^{t} \otimes j^{t} \otimes j^{t}\right) \Phi_{1}=\left(F_{23}(i d \otimes \Delta)(F) \Phi_{2}(\Delta \otimes i d)\left(F^{-1}\right) F_{12}^{-1}\right) .
$$

It follows from equation (49) that

$$
\left(\varphi_{\omega_{2}}(u) \otimes \varphi_{\omega_{2}}(v)\right)\left(j^{t} \otimes j^{t}\right) R_{1}=\left(\varphi_{\omega_{1}}(j(u)) \otimes \varphi_{\omega_{1}}(j(v))\right) R_{1}=d_{\omega_{1}}[j(v) \mid j(u)]
$$

and

$$
\left(\varphi_{\omega_{2}}(u) \otimes \varphi_{\omega_{2}}(v)\right) F_{21} R_{2} F^{-1}=f(v, u) f^{-1}(u, v) d_{\omega_{2}}[v \mid u] .
$$

Hence, by equation (53),

$$
\left(j^{t} \otimes j^{t}\right) R_{1}=F_{21} R_{2} F^{-1} .
$$

Therefore, $j^{t}: D^{\omega_{1}}\left(G_{1}\right) \longrightarrow D^{\omega_{2}}\left(G_{2}\right)_{F}$ is a quasi-bialgebra isomorphism.

Theorem 10.5. Let $G_{1}, G_{2}$ be finite abelian groups and $\omega_{1} \in Z^{3}\left(G_{1}, \mathbb{C}^{*}\right)_{a b}, \omega_{2} \in$ $Z^{3}\left(G_{2}, \mathbb{C}^{*}\right)_{a b}$. Then, $D^{\omega_{1}}\left(G_{1}\right)$ and $D^{\omega_{2}}\left(G_{2}\right)$ are gauge equivalent if, and only if $D^{\omega_{1}}\left(G_{1}\right)$-Mod and $D^{\omega_{2}}\left(G_{2}\right)$-Mod are equivalent additive braided tensor categories; that is, there exists a braided tensor equivalence $\left(\mathcal{F}, \varphi_{0}, \varphi_{2}\right)$ between $D^{\omega_{1}}\left(G_{1}\right)$-Mod and $D^{\omega_{2}}\left(G_{2}\right)$-Mod with $\mathcal{F}$ being additive. 
Proof. The "only if" part of the statement is well-known (cf. Kas95]). Conversely, let $\left(\mathcal{F}, \varphi_{0}, \varphi_{2}\right)$ be an additive braided tensor equivalence from $K$-Mod to $S$-Mod where $K=D^{\omega_{1}}\left(G_{1}\right)$ and $S=D^{\omega_{2}}\left(G_{2}\right)$. By Morita theory (cf. [AF92], Lam99]), $\mathcal{F}$ induces an algebra isomorphism $\alpha$ of $\operatorname{End}_{S}(\mathcal{F}(K))$ with $K$ and so $\mathcal{F}(K)$ becomes a $S$ - $K$-bimodule. Moreover, $\mathcal{F}$ is equivalent to the tensor functor $\mathcal{F}(K) \otimes_{K}$ ?. Note that both $S$ and $K$ are semisimple and their irreducible modules are 1-dimensional. Therefore, $\mathcal{F}(K)={ }_{S} S$ and the right $K$-module action is given by the isomorphism $\alpha^{-1}: K \longrightarrow S$ where $S$ is naturally identified with $\operatorname{End}\left({ }_{S} S\right)$. For any $M \in K$-Mod, define $\alpha^{*}(M)$ to be the left $S$-module with the underlying space $M$ and the left $S$-action given by

$$
s m=\alpha(s) m
$$

for any $s \in S$ and $m \in M$. It is easy to see that

$$
\operatorname{Hom}_{K}(M, N)=\operatorname{Hom}_{S}\left(\alpha^{*}(M), \alpha^{*}(N)\right)
$$

for any $M, N \in K$-Mod. Hence, $\alpha^{*}$ defines an additive functor from $K$-Mod to $S$-Mod. It is straightforward to show that $\alpha^{*}$ and $\mathcal{F}(K) \otimes_{K}$ ? are equivalent functors. Without loss of generality, we may assume $\mathcal{F}=\alpha^{*}$.

Now $\mathbb{C} \stackrel{\varphi_{0}}{\longrightarrow} \alpha^{*}(\mathbb{C})$ is scalar multiplication by a nonzero complex number $\kappa$. For any $M \in S$-Mod, define $\eta_{M}$ to be scalar multiplication $\kappa^{-1}$. Then $\eta: \alpha^{*} \longrightarrow$ $\alpha^{*}$ is a natural isomorphism. Hence, $\left(\alpha^{*}, i d, \kappa \varphi_{2}\right)$ is a braided tensor equivalence from $K$-Mod to $S$-Mod. Consider $\kappa \varphi_{2}(K, K)$. Then $\kappa \varphi_{2}(K, K)\left(1_{K} \otimes 1_{K}\right)$ is an invertible in $K \otimes K$ and we set $F^{-1}=\kappa \varphi_{2}(K, K)\left(1_{K} \otimes 1_{K}\right)$. Then, $F$ is a gauge transform of $K$ (cf. [Kas95], p. 381) and

$$
\begin{aligned}
& \alpha\left(\Phi_{S}\right)=F_{23}\left(i d \otimes \Delta_{K}\right)(F) \Phi_{K}(\Delta \otimes i d)\left(F^{-1}\right) F_{12}^{-1}, \\
& \alpha\left(R_{S}\right)=F_{21} R_{K} F^{-1},
\end{aligned}
$$

where $\Phi_{S}, R_{S}$ and $\Phi_{K}, R_{K}$ are the associators and $\mathcal{R}$-matrices of $S$ and $K$ respectively.

Definition 10.6. Let $\Gamma$ be a finite abelian group and $b: \Gamma \times \Gamma \longrightarrow \mathbb{C}^{*}$ a symmetric bicharacter. For any subset $M$ of $G$, denote by $M^{\perp}$ the subgroup

$$
\{x \in \Gamma \mid b(x, M)=1\}
$$

of $\Gamma$. A subgroup $N$ of $\Gamma$ is called a metabolizer of $(\Gamma, b)$ if $N=N^{\perp}$. A quadratic map $q: \Gamma \longrightarrow \mathbb{C}^{*}$ is called non-degenerate if the associated bicharacter $b_{q}(x, y)=$ $\frac{q(x y)}{q(x) q(y)}$ is non-degenerate. A subgroup $N$ of $\Gamma$ is called a metabolizer of $(\Gamma, q)$ if $\left.q\right|_{N}=1$ and $N$ is a metabolizer of $\left(\Gamma, b_{q}\right)$. A metabolizer $N$ of $(\Gamma, q)$ or $(\Gamma, b)$ is called split if $N$ is a summand of $\Gamma$.

Remark 10.7. (i) Let $G$ be a finite abelian group and $\omega$ a normalized 3-cocycle in $Z^{3}\left(G, \mathbb{C}^{*}\right)_{a b}$. Then, $q_{\omega}(\widehat{G})=1$. Moreover, if $b_{\omega}(\sigma(\alpha, x), \sigma(\lambda, 1))=1$ for all $\lambda \in \widehat{G}$, then $\lambda(x)=1$ for all $\lambda \in \widehat{G}$. This implies $x=1$. Therefore, $\widehat{G}^{\perp}=\widehat{G}$ and hence $\widehat{G}$ is a metabolizer of $\left(\Gamma^{\omega}, q_{\omega}\right)$.

(ii) If $(\Gamma, q)$ is non-degenerate with metabolizer $G$, then $|G|^{2}=|\Gamma|$.

Let $G, G^{\prime}$ be finite abelian groups, $\omega \in Z^{3}\left(G, \mathbb{C}^{*}\right)_{a b}$ and $\omega^{\prime} \in Z^{3}\left(G^{\prime}, \mathbb{C}^{*}\right)_{a b}$. Let $\zeta \in Z^{3}\left(G_{1} \times G_{2}, \mathbb{C}^{*}\right)_{a b}$ be the product of the inflations of $\omega$ and $\omega^{\prime}$, namely,

$$
\zeta=(i n f l \omega)\left(i n f l \omega^{\prime}\right)
$$


where the inflations are induced by the natural surjections $G \times G^{\prime} \longrightarrow G$ and $G \times G^{\prime} \longrightarrow G^{\prime}$. By the remarks following Proposition 4.1, there is an isomorphism of groups $\iota: \Gamma^{\omega}(G) \times \Gamma^{\omega^{\prime}}(G) \longrightarrow \Gamma^{\zeta}\left(G \times G^{\prime}\right)$ given by

$$
\iota\left(\sum_{g \in G} \lambda_{x}(g) e(g) \otimes x, \sum_{h \in G^{\prime}} \lambda_{y}^{\prime}(h) e(h) \otimes y\right)=\sum_{(g, h) \in G \times G^{\prime}} \lambda_{x}(g) \lambda_{y}^{\prime}(h) e(g, h) \otimes(x, y)
$$

where $\delta \lambda_{x}=\omega_{x}, \delta \lambda_{y}^{\prime}=\omega_{y}^{\prime}$ and $x \in G, y \in G^{\prime}$. Since, for any

$$
u=\sum_{(g, h) \in G \times G^{\prime}} \lambda_{(x, y)}(g, h) e(g, h) \otimes(x, y) \in \Gamma^{\zeta}\left(G \times G^{\prime}\right), q_{\zeta}(u)=\lambda_{(x, y)}(x, y),
$$

$\iota$ defines an equivalence of the quadratic forms $\left(\Gamma^{\zeta}, q_{\zeta}\right)$ and $\left(\Gamma^{\omega}, q_{\omega}\right) \perp\left(\Gamma^{\omega^{\prime}}, q_{\omega^{\prime}}\right)$.

Conversely, suppose that $H$ is a finite abelian group and $\eta \in Z^{3}\left(H, \mathbb{C}^{*}\right)_{a b}$ such that $\left(\Gamma^{\eta}, q_{\eta}\right)$ is equivalent to the orthogonal sum $\left(\Gamma^{\omega}, q_{\omega}\right) \perp\left(\Gamma^{\omega^{\prime}}, q_{\omega^{\prime}}\right)$. Then, $\left(\Gamma^{\eta}(H), q_{\eta}\right)$ and $\left(\Gamma^{\zeta}\left(G \times G^{\prime}\right), q_{\zeta}\right)$ are equivalent quadratic forms. By virtue of Proposition 10.4, $D^{\eta}(H)$ is equivalent to $D^{\omega}(H) \otimes D^{\omega^{\prime}}\left(H^{\prime}\right)$ as quasi-triangular quasi-bialgebras. This proves

Proposition 10.8. Let $G$ be a finite abelian group and $\eta \in Z^{3}\left(G, \mathbb{C}^{*}\right)_{a b}$. Then, $D^{\eta}(G)$ is equivalent to $D^{\omega}(H) \otimes D^{\omega^{\prime}}\left(H^{\prime}\right)$ as quasi-triangular quasi-bialgebras for some abelian groups $H, H^{\prime}$ and $\omega \in Z^{3}\left(H, \mathbb{C}^{*}\right)_{a b}, \omega^{\prime} \in Z^{3}\left(H^{\prime}, \mathbb{C}^{*}\right)_{a b}$ if, and only if, $\left(\Gamma^{\eta}, q_{\eta}\right)$ is equivalent to $\left(\Gamma^{\omega}, q_{\omega}\right) \perp\left(\Gamma^{\omega^{\prime}}, q_{\omega^{\prime}}\right)$.

\section{LATTices}

We use the following notation: $M$ is a self-dual, even lattice with respect to the nonsingular bilinear form

$$
\langle\bullet, \bullet\rangle: M \times M \longrightarrow \mathbb{Z} .
$$

Thus $x \mapsto\langle x, \bullet\rangle$ is an isomorphism of $M$ with $\operatorname{Hom}_{\mathbb{Z}}(M, \mathbb{Z})$; moreover, $\langle x, x\rangle$ is an even integer for $x \in M$. Note that $\langle\bullet, \bullet\rangle$ is not necessarily positive definite.

Let $E$ be the space $\mathbb{R} \otimes_{\mathbb{Z}} M$ equipped with the $\mathbb{R}$-linear extension of $\langle\bullet, \bullet\rangle$, let $M \subseteq L \subseteq E$ with $|L: M|\left\langle\infty\right.$, and let $L_{0}=\{x \in E \mid\langle x, L\rangle \subseteq \mathbb{Z}\}$ be the $\mathbb{Z}$-dual of $L$. We set $G=L / M$. There is a short exact sequence

$$
0 \longrightarrow M / L_{0} \longrightarrow L / L_{0} \longrightarrow L / M \longrightarrow 0
$$

and we pick a section $s: L / M \longrightarrow L$, such that $s(0)=0$, which naturally defines a section $\bar{s}: L / M \longrightarrow L / L_{0}$.

Because $M$ is self-dual, the pairing $\langle\bullet, \bullet\rangle: L \times L \longrightarrow \mathbb{Q}$ induces a perfect pairing

$$
p: M / L_{0} \times L / M \longrightarrow S^{1}, \quad\left(x+L_{0}, y+M\right) \mapsto e^{2 \pi i\langle x, y\rangle}
$$

and so there is a natural identification of $M / L_{0}$ with $\operatorname{Hom}\left(L / M, S^{1}\right)=\widehat{G}$. Thus the sequence (54) is of the type we have been considering. That is, the triple $L_{0} \subseteq M \subseteq L$ defines an element of $H_{a b}^{2}(G, \widehat{G})$ with $G=L / M$, and it is wellknown that a 2-cocycle $\beta \in Z_{a b}^{2}(G, \widehat{G})$ which corresponds to the triple is defined, using the section $\bar{s}$, via

$$
\beta(x, y)=\bar{s}(x)+\bar{s}(y)-\bar{s}(x+y)
$$

for $x, y \in G$. 
Following Dong and Lepowsky DL93, we pick an alternating, bilinear map $c: L \times L \longrightarrow S^{1}$ with the property

$$
c(x, y)=(-1)^{\langle x, y\rangle}
$$

for $x, y \in M$. Such $c$ always exists (cf. DL93, Remark 12.18).

Proposition 11.1. With the previous notation, set

$$
\begin{gathered}
\omega(g, x, y)=c(s(g), s(x)+s(y)-s(x+y)) e^{\pi i\langle s(g), s(x)+s(y)-s(x+y)\rangle}, \\
\tau_{g}(x)=c(s(g), s(x)) e^{\pi i\langle s(g), s(x)\rangle} .
\end{gathered}
$$

Then $\omega \in Z^{3}\left(G, \mathbb{C}^{*}\right)_{a b}, \omega_{g}=\delta \tau_{g}$, and $\Lambda_{G}([\omega])=[\beta]$.

Proof. After some computation, we find that for $g, h, x, y \in G$,

$$
\begin{aligned}
\delta \omega(g, h, x, y)= & c(s(g)+s(h)-s(g+h), s(x)+s(y)-s(x+y)) \\
& \cdot e^{\pi i\langle s(g)+s(h)-s(g+h), s(x)+s(y)-s(x+y)\rangle} .
\end{aligned}
$$

Note that both $a=s(g)+s(h)-s(g+h)$ and $b=s(x)+s(y)-s(x+y)$ lie in $M$. Using equation (57), the expression of (60) is equal to 1 and, as a result, $\omega$ is a normalized 3-cocycle.

The identity $\omega_{g}=\delta \tau_{g}$ follows immediately from (58) and (59). Finally, using the expression (15) together with (59) shows that $\Lambda_{G}([\omega])$ is the cohomology class represented by $\beta_{1} \in Z_{a b}^{2}(G, \widehat{G})$ where

$$
\begin{aligned}
& \beta_{1}(x, y)(g) \\
& =\frac{c(s(g), s(x)) c(s(x), s(g)) c(s(g), s(y)) c(s(y), s(g))}{c(s(g), s(x+y)) c(s(x+y), s(g))} e^{2 \pi i\langle s(g), s(x)+s(y)-s(x+y)\rangle}
\end{aligned}
$$

for $x, y, g \in G$. Since $c$ is alternating, equations (55) and (56) yield

$$
\beta_{1}(x, y)(g)=p(\beta(x, y), g) .
$$

So by definition of the pairing $p, \beta_{1}=\beta$.

We now have two quadratic forms associated to this situation: $\left(L / L_{0}, q_{L}\right)$ where $q_{L}\left(x+L_{0}\right)=e^{\pi i\langle x, x\rangle}$ for $x \in L$; and $\left(\Gamma^{\omega}, q_{\omega}\right)$ canonically associated to the 3 -cocycle $\omega($ cf. (48) $)$.

Lemma 11.2. These two quadratic spaces are equivalent.

Proof. Let notation be as before. From section $3 \Gamma^{\omega}$ is a central extension of $G$ by $\widehat{G}$. The additive version of the corresponding 2 -cocycle $\beta$ is given by (56). Here, as before, $G=L / M$ and $\hat{G}=M / L_{0}$ under the identification given in (55). There is an isomorphism $j: L / L_{0} \longrightarrow \Gamma^{\omega}$ defined for $x \in L$ via

$$
x+L_{0} \mapsto \sigma\left(x+L_{0}-\bar{s}(x+M), x+M\right) .
$$

Now use equations (48), (59) to see that

$$
q_{\omega}\left(j\left(x+L_{0}\right)\right)=p\left(x+L_{0}-\bar{s}(x+M), x+M\right) e^{\pi i\langle s(x), s(x)\rangle} .
$$

Write $s(x+M)=x+m$ for some $m \in M$. Then we see that

$$
\begin{aligned}
q_{\omega}\left(j\left(x+L_{0}\right)\right) & =e^{\pi i(2\langle x-(x+m), x\rangle+\langle x+m, x+m\rangle)}, \\
& =e^{\pi i\langle x, x\rangle}=q_{L}\left(x+L_{0}\right) .
\end{aligned}
$$

This completes the proof of the lemma. 
We now begin again, this time with a non-degenerate quadratic form $q$ on the finite abelian group $\Gamma$. It is convenient to write $q$ additively, that is, $q: \Gamma \longrightarrow \mathbb{Q} / \mathbb{Z}$. We also consider $q_{1}=2 q$ as a quadratic form with values in $\mathbb{Q} / 2 \mathbb{Z}$. According to Wal63, Theorem (6), there is a rational lattice $\left(H^{\prime},\langle\bullet, \bullet\rangle\right)$ i.e. $\langle\bullet, \bullet\rangle: H^{\prime} \times H^{\prime} \longrightarrow$ $\mathbb{Q}$ non-degenerate, an even (integral) sublattice $H \subseteq H^{\prime}$, and an equivalence of quadratic forms

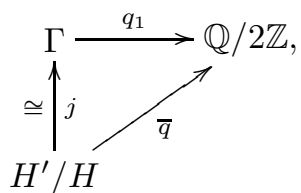

where $\bar{q}$ is the quadratic form arising from $\langle\bullet, \bullet\rangle$. Thus, for $x, y \in H^{\prime}$ we have $\bar{q}(x+H)=\langle x, x\rangle(\bmod 2 \mathbb{Z})$ and

$$
\begin{aligned}
q_{1}(j(x+H)) & \equiv\langle x, x\rangle \quad(\bmod 2 \mathbb{Z}), \\
b(j(x+H), j(y+H)) & \equiv\langle x, y\rangle \quad(\bmod \mathbb{Z})
\end{aligned}
$$

where $b$ is the bilinear form on $\Gamma$ associated to $q$.

Now assume that $(\Gamma, q)$ has a metabolizer $\widehat{G}$, and write $j^{-1}(\widehat{G})=M / H$ for a sublattice $M$ with $H \subseteq M \subseteq H^{\prime}$. Since $\widehat{G}^{\perp}=\widehat{G}$, it follows from (64) that $M$ is a self-dual, integral sublattice of $H^{\prime}$. Similarly, equation (63) shows that $M$ is an even lattice. We now have

Theorem 11.3. (i) Let $M \subseteq L$ be a pair of rational lattices with $M$ even and self-dual, and let $L_{0} \subseteq M$ be the $\mathbb{Z}$-dual of $L$. Then the pair $\left(L / L_{0}, q_{L}\right)$ is a non-degenerate quadratic space with metabolizer $M / L_{0}$.

(ii) Furthermore, there is $\omega \in Z^{3}\left(G, \mathbb{C}^{*}\right)_{a b}$ such that the corresponding quadratic space $\left(\Gamma^{\omega}, q_{\omega}\right)$ is equivalent to $\left(L / L_{0}, q_{L}\right)$. Moreover, $\Lambda(\omega)$ is cohomologous to the element of $Z_{a b}^{2}\left(L / M, M / L_{0}\right)$ defined by (56).

(iii) Conversely, suppose that $(\Gamma, q)$ is a non-degenerate quadratic space with metabolizer. Then there are lattices $M \subseteq L$ as in (i) such that $(\Gamma, q)$ and $\left(L / L_{0}, q_{L}\right)$ are equivalent quadratic spaces.

(iv) Every non-degenerate quadratic space $(\Gamma, q)$ with a metabolizer $\widehat{G}$ is equivalent to one of the form $\left(\Gamma^{\omega}, q_{\omega}\right)$ for suitable $\omega \in Z^{3}\left(G, \mathbb{C}^{*}\right)_{a b}$.

Proof. (i) and (ii) follow from Proposition 11.1 and Lemma 11.2, while (iii) follows from the discussion following the proof of the Lemma. Part (iv) follows from (i)(iii).

Remark 11.4. From Theorem 11.3 and our earlier work, we see that the following pieces of data are more or less equivalent:

1. A pair of rational lattices $M \subseteq L$ with $M$ even and self-dual, $L / M \cong G$.

2. A non-degenerate quadratic space $(\Gamma, q)$ with metabolizer $\widehat{G}$.

3. A cohomology class $[\omega] \in H^{3}\left(G, \mathbb{C}^{*}\right)_{a b}$.

4. A cohomology class $[\beta] \in H_{a b}^{2}(G, \widehat{G})$ which is in the image of $S$ (cf. Theorem 8.4.

\section{Gauss sums}

We begin with a discussion of Gauss sums on pairs $(M, q)$ consisting of a finite abelian group $M$ and a non-degenerate quadratic form $q: M \longrightarrow \mathbb{Q} / \mathbb{Z}$. Thus, 
$q(x+y)=q(x)+q(y)+b(x, y)$ where $b: M \times M \longrightarrow \mathbb{Q} / \mathbb{Z}$ is a non-degenerate bilinear form. In our applications, $(M, q)$ will be the pair $\left(\Gamma^{\omega}, q_{\omega}\right)$ that we have already considered, or something related to it. Our discussion is closely related to that of tom Dieck (tD79, 2.2) adapted to our present needs.

The Gauss sum $G(M, q)$ is defined via

$$
G(M, q)=\sum_{m \in M} e^{2 \pi i q(m)} .
$$

The Gauss man $\gamma$ is defined via

$$
\gamma(M, q)=\frac{1}{\sqrt{|M|}} G(M, q) .
$$

Lemma 12.1. Let $N \subseteq M$ be a subgroup of $M$ such that the restriction of $q$ to $N$ vanishes identically. Then $q$ induces a non-degenerate quadratic form $\bar{q}$ on $N^{\perp} / N$, and we have

$$
\gamma(M, q)=\gamma\left(N^{\perp} / N, \bar{q}\right)
$$

Proof. Note that $\bar{q}$ is defined via $\bar{q}(m+N)=q(m)$ for $m \in N^{\perp}$. It is easy to see that this is well-defined and yields a quadratic form on $N^{\perp} / N$ which is non-degenerate. Then we calculate

$$
\begin{aligned}
\gamma(M, q) & =\frac{1}{\sqrt{|M|}} \frac{1}{|N|} \sum_{n \in N} \sum_{m \in M} e^{2 \pi i q(m+n)} \\
& =\frac{1}{\sqrt{|M|}} \frac{1}{|N|} \sum_{m \in M} e^{2 \pi i q(m)} \sum_{n \in N} e^{2 \pi i b(m, n)}
\end{aligned}
$$

where we used $q(m+n)=q(m)+q(n)+b(m, n)$ and $q(n)=0$ for $n \in N$. Now the inner sum vanishes whenever $m \notin N^{\perp}$ by the orthogonality of group characters, and is otherwise equal to $|N|$. So in fact

$$
\begin{aligned}
\gamma(M, q) & =\frac{1}{\sqrt{|M|}} \sum_{m \in N^{\perp}} e^{2 \pi i q(m)} \\
& =\frac{|N|}{\sqrt{|N|\left|N^{\perp}\right|}} \sum_{m \in N^{\perp} / N} e^{2 \pi i \bar{q}(m)} \\
& =\gamma\left(N^{\perp} / N, \bar{q}\right) .
\end{aligned}
$$

Corollary 12.2. If $(M, q)$ has a metabolizer, then $\gamma(M, q)=1$.

Lemma 12.3. Let $p$ be a prime and $(M, q)$ a non-degenerate quadratic space on the non-trivial cyclic p-group $M$. One of the following holds:

(i) $p$ is odd, $|M|$ is a square, and $(M, q)$ has a metabolizer.

(ii) $p \equiv 1(\bmod 4),|M|$ is not a square, and $\gamma(M, q)= \pm 1$.

(iii) $p \equiv 3(\bmod 4),|M|$ is not a square, and $\gamma(M, q)= \pm i$.

(iv) $p=2$ and $\gamma(M, q)$ is a primitive 8th root of unity.

\footnotetext{
${ }^{5}$ We say map because $\gamma$ can be considered as a function on a suitable Witt group.
} 
Proof. Pick $N \subseteq M$ maximal subject to the condition that the restriction of $q$ to $N$ is identically zero. Set $V=N^{\perp} / N$, so that $\gamma(M, q)=\gamma(V, \bar{q})$ as in Lemma 12.1] Now the maximality of $N$ implies that $(V, \bar{q})$ is anisotropic, that is, if $v \in V$ and $\bar{q}(v)=0$ then in fact $v=0$. It is then easy to see that $|V| \leq p^{2}$, and even $|V| \leq p$ if $p$ is odd. First assume that $p$ is odd. Then (i) holds if $V=0$, and otherwise $|V|=p$ and $|M|=|N|^{2}|V|$ is not a square. Then (ii) or (iii) holds by the classical calculation of Gauss sums (cf. to79]).

Now take $p=2$. If $|V|=2$ or 4 it is an easy direct calculation that (iv) holds. To complete the lemma, we must show that, in fact, $V \neq 0$ if $p=2$. Assume, by way of contradiction, that indeed $V=0$. Then $|M|=|N|^{2}=2^{2 n}$, say. Let $x$ be a generator of $M$. Then $2^{n} x$ generates $N$ and therefore we have $q\left(2^{n} x\right)=0$. As $b$ is non-degenerate, $b(x, x)=k / 2^{2 n}$ for some odd integer $k$. It follows from the equality

$$
q(2 x)-2 q(x)=b(x, x)
$$

that $q(x)=k^{\prime} / 2^{n+1}$ for some odd integer $k^{\prime}$. Hence $q\left(2^{n} x\right)=k^{\prime} / 2 \neq 0$, contradiction.

Now consider a non-degenerate pair $(M, q)$ which has a metabolizer, and where $M$ is a $p$-group with $p \equiv 1(\bmod 4)$. We may write $M$ as an orthogonal direct sum of pairs $\left(M_{i}, q_{i}\right)$ with $M_{i}$ cyclic (Wal63], Theorem 4). By Lemma 12.3, if $\left|M_{i}\right|$ is a square, then $\left(M_{i}, q_{i}\right)$ has a metabolizer and $\gamma\left(M_{i}, q_{i}\right)=1$. Since $|M|$ is a square (by Remark 10.7(ii)) and $\gamma(M, q)=1$, there must be an even number of $M_{i}$ whose order is not a square and satisfy $\gamma\left(M_{i}, q_{i}\right)=-1$, and an even number of $M_{i}$ whose order is not a square and satisfy $\gamma\left(M_{i}, q_{i}\right)=1$. So we see that now we may write (with a change of notation)

$$
(M, q)=\left(M_{1}, q_{1}\right) \perp \cdots \perp\left(M_{r}, q_{r}\right)
$$

with $\gamma\left(M_{i}, q_{i}\right)=1$ for each $i$, and $M_{i}$ is either cyclic of square order or the product of two cyclic groups, each of non-square order.

Before we proceed further with our discussion, let us recall Wall's nomenclature for non-degenerate symmetric bilinear forms on a finite cyclic group. Let $G$ be a cyclic group of order $p^{n}$ with generator $x$, where $p$ is an odd prime. A bilinear form $b: G \times G \longrightarrow \mathbb{Q} / \mathbb{Z}$ with $b(x, x)=\epsilon p^{-n}$ is of type $A$ if $\epsilon=1$, and it is of type $B$ if $\epsilon$ is a quadratic non-residue modulo $p$ (cf. Wal63).

We assert that each $\left(M_{i}, q_{i}\right)$ in (65) has a cyclic metabolizer. This has already been explained if $M_{i}$ is cyclic. Otherwise, $M_{i}=H \oplus K$ with $H, K$ both nonzero cyclic groups and $\gamma\left(H, q_{i}\right) \gamma\left(K, q_{i}\right)=1$. Then $\gamma\left(H, q_{i}\right)=\gamma\left(K, q_{i}\right)$ and from section 5 of Wal63] we see that $H$ and $K$ are either both of type A or both of type B. More precisely, there are generators $x, y$ of $H, K$ respectively (and of order $p^{h}$, $p^{k}$ respectively, say) such that $b_{i}(x, x)=\epsilon p^{-h}$ and $b_{i}(y, y)=-\epsilon p^{-k}$ where $\epsilon$ is either 1 or a non-residue $(\bmod p)$. (Here we have used the fact that $\left(\frac{-1}{p}\right)=1$ for $p \equiv 1(\bmod 4)$.$) Now if h=r-u$ and $k=r+u$ for nonnegative integers $r, u$ we find that the subgroup generated by $x+p^{u} y$ is the metabolizer we require.

We can rewrite (65) in terms of twisted quantum doubles using Proposition 10.8 As each $\left(M_{i}, q_{i}\right)$ has a cyclic metabolizer $G_{i}$, say, then we know (cf. Remark 11.4) that the pair $\left(M_{i}, q_{i}\right)$ can be realized via $D^{\omega_{i}}\left(G_{i}\right)$ for suitable $\omega_{i} \in Z^{3}\left(G, \mathbb{C}^{*}\right)_{a b}$. That is, $\left(M_{i}, q_{i}\right)$ is equivalent to $\left(\Gamma^{\omega_{i}}, q_{\omega_{i}}\right)$. The orthogonal sum (65) corresponds 
to the tensor product of twisted doubles. So if we start with a pair $(G, \omega)$ which gives rise to $(M, q)$, we can conclude

Theorem 12.4. Suppose that $p \equiv 1(\bmod 4)$ is a prime, $G$ an abelian $p$-group, and $\omega \in Z^{3}(G, \mathbb{C})_{a b}$. Then there are cyclic p-groups $G_{i}$ and cocycles $\omega_{i} \in Z^{3}\left(G_{i}, \mathbb{C}\right)_{a b}$, $1 \leq i \leq r$, such that the two quasi-triangular, quasi-Hopf algebras $D^{\omega}(G)$ and $\bigotimes_{i=1}^{r} D^{\omega_{i}}\left(G_{i}\right)$ are gauge equivalent.

Remark 12.5. It is evident from our proof that the choice of the isomorphism types of the $G_{i}$ are far from unique. For example, suppose that $M$ is the orthogonal sum of four cyclic group of orders $p, p^{3}, p^{5}, p^{7}$, and each of type A. Then our proof shows that $D^{\omega}(G)$ is equivalent to $D^{\omega_{1}}\left(G_{1}\right) \otimes D^{\omega_{2}}\left(G_{2}\right)$, as quasi-triangular quasibialgebras, where $G_{1}, G_{2}$ can be chosen to be have orders $p^{2}, p^{6} ; p^{3}, p^{5}$; or $p^{4}, p^{4}$. Of course, the number $r$ of tensor factors that occur depends only on $(G, \omega)$, and we always have $|G|=\prod_{i=1}^{r}\left|G_{i}\right|$.

Theorem 12.4 fails for primes not congruent to $1(\bmod 4)$. To treat this case we need

Lemma 12.6. Let $(M, q)$ be a non-degenerate quadratic space on the p-group $M$. Suppose that $|M|$ is a square. Then $\gamma(M, q)=1$ if, and only if, $(M, q)$ has a metabolizer.

Proof. Let $N$ and $V$ be as in the proof of Lemma 12.3 After Corollary 12.2 we may assume $\gamma(M, q)=1$ and $V \neq 0$ and try to reach a contradiction.

We see that $V$ is not cyclic by Lemma 12.3, and since $V$ is anisotropic, then $V \cong \mathbb{Z}_{p} \oplus \mathbb{Z}_{p}$, as is easily verified. From Lemma 12.3 (and its proof), we see that $\gamma(M, q)=-1$ if either $p$ is odd, or if $p=2$ and $V$ is not an orthogonal sum of two cyclic groups. Otherwise, $p=2, V=\langle x, y\rangle, b(x, y)=0, q(x)=q(y)= \pm 1 / 4$, $q(x+y)=1 / 2$. Hence $\gamma(M, q)= \pm i$ in this case.

Now consider the analogue of 65 in the case $p \equiv 3(\bmod 4)$. The arguments preceding (65) together with Lemma 12.3 show that we may still write $(M, q)$ in the form of equation (65), with each $\gamma\left(M_{i}, q_{i}\right)=1$, and $\left|M_{i}\right|$ a square. However, $M_{i}$ may now be a product of 1,2 or 4 cyclic subgroups. By Lemma 12.6, each $\left(M_{i}, q_{i}\right)$ has a metabolizer. Thus there is an analogue of Theorem 12.4 for $p \equiv 3(\bmod 4)$, except that the groups $G_{i}$ cannot necessarily be chosen to be cyclic. Rather, we have the property that the group $\Gamma^{\omega_{i}}\left(G_{i}\right)$ is a product of 1,2 or 4 cyclic groups.

Finally, take $p=2$, and assume again that $\gamma(M, q)=1$. We can decompose $(M, q)$ as follows ([Wal63], sections 5 and 6)

$$
(M, q)=\left(P_{1}, q_{1}\right) \perp \cdots \perp\left(P_{t}, q_{t}\right)
$$

with each $P_{i}$ homogeneous and the product of at most 2 cyclic groups. From Lemma 12.3, and the proof of Lemma 12.6, we see that the number of cyclic factors in (66) is necessarily even. Hence $M$ is the product of an even number of cyclic groups, which thus gives a different proof of Theorem 8.5. The analogue of Theorem 12.4 for $p=2$ is the following: $D^{\omega}(G)$ is gauge equivalent to $\bigotimes_{i=1}^{r} D^{\omega_{i}}\left(G_{i}\right)$ and for each $i, \Gamma^{\omega_{i}}\left(G_{i}\right)$ is the product of $1,2,4$ or 8 cyclic groups.

\section{Metabolic Forms And homogeneous groups}

A metabolic form is a triple $(\Gamma, b, G)$ with $b: \Gamma \times \Gamma \longrightarrow \mathbb{Q} / \mathbb{Z}$ a non-degenerate symmetric bilinear form and $G$ a metabolizer of $(\Gamma, b)$. If $\Gamma^{\prime} \leq \Gamma$ and $G^{\prime} \leq G$ such 
that $\left(\Gamma^{\prime}, b, G^{\prime}\right)$ also a metabolic form, then $\left(\Gamma^{\prime}, b, G^{\prime}\right)$ is called a sub-metabolic form of $(\Gamma, b, G)$. Obviously, $(\{0\}, b,\{0\})$ is a trivial sub-metabolic form of $(\Gamma, b, G)$. A metabolic form is called simple if $(\Gamma, b, G)$ is the only nontrivial sub-metabolic form.

A group homomorphism $j: \Gamma_{1} \longrightarrow \Gamma_{2}$ is called a morphism mapping $\left(\Gamma_{1}, b_{1}, G_{1}\right)$ to $\left(\Gamma_{2}, b_{2}, G_{2}\right)$ if $j\left(G_{1}\right) \subseteq G_{2}$ and

$$
b_{2}(j(u), j(v))=b_{1}(u, v)
$$

for any $u, v \in \Gamma_{1}$. If the morphism $j$ is bijective, then we call the two metabolic forms equivalent and denote this by

$$
\left(\Gamma_{1}, b_{1}, G_{1}\right) \cong\left(\Gamma_{2}, b_{2}, G_{2}\right) .
$$

Proposition 13.1. Let $G, G^{\prime}$ be finite abelian groups of odd order, $\omega \in Z^{3}\left(G, \mathbb{C}^{*}\right)_{a b}$ and $\omega^{\prime} \in Z^{3}\left(G^{\prime}, \mathbb{C}^{*}\right)_{a b}$. Then $\left(\Gamma^{\omega}(G), b_{\omega}, \widehat{G}\right) \cong\left(\Gamma^{\omega^{\prime}}\left(G^{\prime}\right), b_{\omega^{\prime}}, \widehat{G}^{\prime}\right)$ if, and only if, $\omega^{\prime}$ and $\widehat{\rho} w$ are cohomologous for some isomorphism $\rho: G \longrightarrow G^{\prime}$ where $\widehat{\rho} \omega$ is as in Remark 2.1(iii).

Proof. Let $\rho: G \longrightarrow G^{\prime}$ be an isomorphism such that $\widehat{\rho} \omega=\omega^{\prime} \delta b^{\prime}$ for some normalized 2-cochain $b^{\prime}$ on $G^{\prime}$. Let $\tau \in T(\omega)$. Then $\tau^{\prime} \in T\left(\omega^{\prime}\right)$ where

$$
\tau_{x^{\prime}}^{\prime}\left(y^{\prime}\right)=\tau_{\rho^{-1} x^{\prime}}\left(\rho^{-1} y^{\prime}\right) \frac{b^{\prime}\left(y^{\prime}, x^{\prime}\right)}{b^{\prime}\left(x^{\prime}, y^{\prime}\right)}
$$

for any $x^{\prime}, y, \in G^{\prime}$. The map $j: D^{\omega}(G) \longrightarrow D^{\omega^{\prime}}\left(G^{\prime}\right)$ defined by

$$
j: e(g) \otimes x \mapsto \frac{b^{\prime}(\rho g, \rho x)}{b^{\prime}(\rho x, \rho g)} e(\rho g) \otimes \rho x
$$

is a bialgebra isomorphism. In particular, $j\left(\Gamma^{\omega}(G)\right)=\Gamma^{\omega^{\prime}}\left(G^{\prime}\right)$. Moreover, for any $\sigma(\alpha, x) \in \Gamma^{\omega}(G)$

$$
j(\sigma(\alpha, x))=\sum_{g \in G} \alpha(g) \tau_{x}(g) \frac{b^{\prime}(\rho g, \rho x)}{b^{\prime}(\rho x, \rho g)} e(\rho g) \otimes \rho x
$$

and so

$$
q_{\omega^{\prime}}(j(\sigma(\alpha, x)))=\alpha(x) \tau_{x}(x)=q_{\omega}(\sigma(\alpha, x)) .
$$

It is obvious that $j(\widehat{G})=\widehat{G^{\prime}}$ and hence $j$ is an isomorphism of $\left(\Gamma^{\omega}(G), b_{\omega}, \widehat{G}\right)$ with $\left(\Gamma^{\omega^{\prime}}\left(G^{\prime}\right), b_{\omega^{\prime}}, \widehat{G}^{\prime}\right)$.

Conversely, assume that $j$ is an isomorphism of $\left(\Gamma^{\omega}(G), b_{\omega}, \widehat{G}\right)$ with $\left(\Gamma^{\omega^{\prime}}\left(G^{\prime}\right), b_{\omega^{\prime}}, \widehat{G}^{\prime}\right)$. For $x \in G$,

$$
j \sigma_{\tau}(1, x)=\sigma_{\tau^{\prime}}\left(b_{x}, \rho x\right)
$$

for some $b_{x} \in \widehat{G}^{\prime}$ and $\rho x \in G$. As $j(\widehat{G})=\widehat{G}^{\prime}$, the map $\rho: x \mapsto \rho x$ defines an isomorphism from $G$ to $G^{\prime}$. As

$$
b_{\omega^{\prime}}\left(\sigma_{\tau^{\prime}}\left(b_{x}, \rho x\right), \sigma_{\tau^{\prime}}\left(b_{y}, \rho y\right)\right)=b_{\omega}\left(\sigma_{\tau}(1, x), \sigma_{\tau}(1, y)\right),
$$

we have the equality

$$
b_{x}(y) b_{y}(x) \tau_{\rho x}^{\prime}(\rho y) \tau_{\rho y}^{\prime}(\rho x)=\tau_{x}(y) \tau_{y}(x) .
$$


Hence,

$$
\begin{aligned}
\frac{\tau_{x}(z) \tau_{y}(z)}{\tau_{x y}(z)} \omega_{z}(x, y) & =\frac{\tau_{x}(z) \tau_{z}(x) \tau_{y}(z) \tau_{z}(y)}{\tau_{x y}(z) \tau_{z}(x y)} \\
& =\frac{\tau_{\rho x}^{\prime}(\rho z) \tau_{\rho z}^{\prime}(\rho x) \tau_{\rho y}^{\prime}(\rho z) \tau_{\rho z}^{\prime}(\rho y)}{\tau_{\rho(x y)}^{\prime}(\rho z) \tau_{\rho z}^{\prime}(\rho(x y))} \frac{b_{x}(z) b_{y}(z)}{b_{x y}(z)} \\
& =\frac{\tau_{\rho x}^{\prime}(\rho z) \tau_{\rho y}^{\prime}(\rho z)}{\tau_{\rho(x y)}^{\prime}(\rho z)} \omega_{\rho z}^{\prime}(\rho x, \rho y)(\delta b)(x, y)(z)
\end{aligned}
$$

where $b \in C^{1}(G, \widehat{G})$ given by $b(x, y)=b_{x}(y)$. By equation (67) and (15), we see that $\Lambda(\widehat{\rho} \omega)$ and $\Lambda\left(\omega^{\prime}\right)$ are cohomologous. As $|G|$ is odd, it follows from Corollary 6.4 that $[\widehat{\rho} \omega]=\left[\omega^{\prime}\right]$.

For two metabolic forms $\left(\Gamma_{1}, b_{1}, G_{1}\right)$ and $\left(\Gamma_{2}, b_{2}, G_{2}\right)$, one can define the orthogonal sum, $\left(\Gamma_{1}, b_{1}, G_{1}\right) \perp\left(\Gamma_{2}, b_{2}, G_{2}\right)$ to be the metabolic form $\left(\Gamma_{1} \times G_{2}, b, G_{1} \times G_{2}\right)$ where

$$
b\left(\left(u_{1}, v_{1}\right),\left(u_{2}, v_{2}\right)\right)=b_{1}\left(u_{1}, v_{1}\right)+b_{2}\left(u_{2}, v_{2}\right)
$$

for any $u_{1}, u_{2} \in G_{1}$ and $v_{1}, v_{2} \in G_{2}$.

Proposition 13.2. Let $(\Gamma, b, G)$ be a metabolic form and $\left(K,\left.b\right|_{K}, H\right)$ a submetabolic form of $(\Gamma, b, G)$. Then, $\left(K^{\perp},\left.b\right|_{K^{\perp}}, G \cap K^{\perp}\right)$ is also a sub-metabolic form of $(\Gamma, b, G)$. Moreover,

$$
(\Gamma, b, G) \cong\left(K,\left.b\right|_{K}, H\right) \perp\left(K^{\perp},\left.b\right|_{K^{\perp}}, G \cap K^{\perp}\right) .
$$

Proof. As $b$ is non-degenerate on $K$, there exists a subgroup $Q<K^{\perp}$ such that $K+G=K+Q$. So for any $x \in G, x=u+v$ for some $u \in K$ and $v \in Q$. Now $H \subseteq G$ and $b(v, H)=0$. This implies $b(u, H)=0$. Since $H$ is a metabolizer of $\left(K,\left.b\right|_{K}\right), u \in H$, it follows that $v \in G$ and hence $G=H+\left(K^{\perp} \cap G\right)$. Moreover,

$$
\left(G \cap K^{\perp}\right)^{\perp} \cap K^{\perp}=(G+K) \cap K^{\perp}=\left(K+\left(K^{\perp} \cap G\right)\right) \cap K^{\perp}=G \cap K^{\perp} .
$$

Hence $G \cap K^{\perp}$ is a metabolizer of $\left(K^{\perp},\left.b\right|_{K^{\perp}}\right)$. It is straightforward to show that

$$
\left(K,\left.b\right|_{K}, H\right) \cong\left(\Gamma,\left.b\right|_{\Gamma}, G\right) \perp\left(K^{\perp},\left.b\right|_{K^{\perp}}, G \cap K^{\perp}\right) \cong(\Gamma, b, G)
$$

with the morphism $j: K \times K^{\perp} \longrightarrow \Gamma$ given by $j(u, v)=u+v$ for any $(u, v) \in$ $K \times K^{\perp}$.

Proposition 13.3. Every metabolic form is equivalent to an orthogonal sum of simple metabolic forms.

Proof. The result follows directly from Proposition 13.2 by induction.

Remark 13.4. It follows easily from Proposition 13.2 that a metabolic form $(\Gamma, b, G)$ is simple if $G$ is cyclic.

We will denote by $\left(K,\left.b\right|_{K}, H\right)^{\perp}$ the metabolic form $\left(K^{\perp},\left.b\right|_{K^{\perp}}, K^{\perp} \cap G\right)$ for any sub-metabolic form $\left(K,\left.b\right|_{K}, H\right)$ of $(\Gamma, b, G)$.

Lemma 13.5. Let $(\Gamma, b, G)$ be a metabolic form with $G$ homogeneous of exponent $p^{n}$. For any non-degenerate cyclic subgroup $D$ of $\Gamma$ of order greater than $p^{n}$, there exists a cyclic subgroup $H \subseteq G$ such that $\left(H+D,\left.b\right|_{H+D}, H\right)$ is a sub-metabolic 
form of $(\Gamma, b, G)$. Moreover, for any generator $y$ of $D$, there is a generator $x$ of $H$ satisfying

$$
x=x^{\prime}+p^{a} y
$$

for some $x^{\prime} \in D^{\perp}$ where $|D|=p^{n+a}$.

Proof. Let $D$ be as in the statement of the lemma. Set $G_{0}=G \cap D^{\perp}$. Then $G / G_{0}$ is cyclic since the map $g+G_{0} \mapsto b(g, \bullet)$ defines an embedding from $G / G_{0}$ into $\widehat{D}$. If $\left|G: G_{0}\right|<p^{n}$, then $\Omega_{p}(G) \subseteq G_{0}$. Therefore, $\Omega_{p}(G) \cap D$ is trivial and so is $G \cap D$. But this implies $|D| \leq p^{n}$ since

$$
D /(G \cap D) \cong(G+D) / G \subseteq \Gamma / G \cong \widehat{G} .
$$

Hence $\left|G: G_{0}\right|=p^{n}$. Then, $G=G_{0} \oplus H$ for some $H \leq G$ isomorphic to $\mathbb{Z}_{p^{n}}$. $H$ can be chosen to contain $G \cap D$ as $G$ is homogeneous. In particular, we have

$$
G \cap D=H \cap D .
$$

Set $K=H+D$. Then $G_{0} \subseteq K^{\perp}$. Therefore,

$$
K^{\perp} \cap K=\left(K+K^{\perp}\right)^{\perp} \subseteq\left(D+H+G_{0}\right)^{\perp}=G^{\perp} \cap D^{\perp}=G_{0} .
$$

As $G \cap K=H+(G \cap D)=H$ by equation (68), $G_{0} \cap K$ is trivial. Hence, $K^{\perp} \cap K=\{0\}$, i.e. $\left(K,\left.b\right|_{K}\right)$ is non-degenerate. Moreover,

$$
H^{\perp} \cap K=H+\left(H^{\perp} \cap D\right)=H+\left(\left(G_{0}+H\right)^{\perp} \cap D\right)=H+(G \cap D) .
$$

By (68), $H^{\perp} \cap K=H$ and hence $H$ is a metabolizer of $\left(K,\left.b\right|_{K}\right)$.

Let $\langle x\rangle=H$ and $\langle y\rangle=D$. Then, $x=x^{\prime}+s p^{a} y$ for some integer $s$ and $x^{\prime} \in D^{\perp}$. If $p \mid s, b\left(p^{n-1} x, y\right)=0$ and so $p^{n-1} x \in G_{0} \cap H$ which contradicts $\operatorname{ord}(x)=p^{n}$. So there is $s^{\prime} \in \mathbb{Z}$ such that $s s^{\prime} \equiv 1(\bmod p)$. The result follows if $x$ is replaced by $s^{\prime} x$.

Lemma 13.6. Let $p$ be an odd prime and $\left(\Gamma, b, G_{1}\right),\left(\Gamma, b, G_{2}\right)$ metabolic forms with $G_{1}$ and $G_{2}$ isomorphic to $\left(\mathbb{Z}_{p^{n}}\right)^{k}$ for some positive integer $k$. Then, there exist non-trivial sub-metabolic forms $\left(K_{i},\left.b\right|_{K_{i}}, H_{i}\right)$ of $\left(\Gamma, b, G_{i}\right), i=1,2$ such that

$$
\left(K_{1},\left.b\right|_{K_{1}}, H_{1}\right) \cong\left(K_{2},\left.b\right|_{K_{2}}, H_{2}\right) .
$$

Proof. Pick $D \leq \Gamma$ cyclic of maximal order such that $\left.b\right|_{D}$ is non-degenerate (cf. Wal63). Then $|D| \geq p^{n}$. If $|D|=p^{n}, G_{1}$ and $G_{2}$ are split metabolizers of $(\Gamma, b)$. It is straightforward to show that there exist $x_{i} \in G_{i}$ and $u_{i}, v_{i} \in \Gamma$ such that $b\left(u_{i}, u_{i}\right)=1 / p^{n}, b\left(v_{i}, v_{i}\right)=-1 / p^{n}, b\left(u_{i}, v_{i}\right)=0$ and $x_{i}=u_{i}+v_{i}$ (cf. [MH73]). In particular, $\left(K_{i},\left.b\right|_{K_{i}},\left\langle x_{i}\right\rangle\right)$ is a sub-metabolic form of $\left(\Gamma, b, G_{i}\right)$ where $K_{i}$ is the subgroup generated by $u_{i}, v_{i}$. Moreover, the map $K_{1} \longrightarrow K_{2}, u_{1} \mapsto u_{2}$ and $v_{1} \mapsto v_{2}$ defines an isomorphism of $\left(K_{1},\left.b\right|_{K_{1}},\left\langle x_{1}\right\rangle\right)$ with $\left(K_{2},\left.b\right|_{K_{2}},\left\langle x_{2}\right\rangle\right)$.

Let $y$ be a generator of $D$ and $\operatorname{ord}(y)=p^{n+a}$ for some positive integer $a$. By Lemma 13.5 for $i=1,2$ there exists $H_{i} \leq G_{i}$ such that $\left(K_{i},\left.b\right|_{K_{i}}, H_{i}\right)$ is a submetabolic form of $\left(\Gamma, b, G_{i}\right)$ where $K_{i}=H_{i}+D$. Moreover, $H_{i}$ admits a generator $x_{i}$ satisfying

$$
x_{i}=x_{i}^{\prime}+p^{a} y
$$

for some $x_{i}^{\prime} \in D^{\perp}$. The map $K_{1} \longrightarrow K_{2}$, given by $y \mapsto y$ and $x_{1}^{\prime} \mapsto x_{2}^{\prime}$, defines an isomorphism of $\left(K_{1},\left.b\right|_{K_{1}}, H_{1}\right)$ with $\left(K_{2},\left.b\right|_{K_{2}}, H_{2}\right)$.

Theorem 13.7. Let $p$ be an odd prime and $(\Gamma, b, G)$ a metabolic form with $G \cong$ $\left(\mathbb{Z}_{p^{n}}\right)^{k}$. 
(i) $(\Gamma, b, G)$ is equivalent to an orthogonal sum of sub-metabolic forms with metabolizers isomorphic to $\mathbb{Z}_{p^{n}}$.

(ii) Let $\left(\Gamma^{\prime}, b^{\prime}, G^{\prime}\right)$ be a metabolic form such that $G^{\prime} \cong G$. Then $\left(\Gamma^{\prime}, b^{\prime}, G^{\prime}\right) \cong$ $(\Gamma, b, G)$ if, and only if $\left(\Gamma^{\prime}, b^{\prime}\right)$ and $(\Gamma, b)$ are equivalent.

Proof. Statement (i) follows easily from Lemma 13.6 and Proposition 13.2 by induction on $k$. For statement (ii), it is obvious that the equivalence of two metabolic forms implies the equivalence of the underlying bilinear forms. Conversely, we proceed by induction on $k$. Let $j$ be an isomorphism of $\left(\Gamma^{\prime}, b^{\prime}, G^{\prime}\right)$ with $(\Gamma, b, G)$. Then, $\left(\Gamma, b, j\left(G^{\prime}\right)\right)$ is a metabolic form. By Lemma 13.6 there exist a nontrivial submetabolic form $\left(K^{\prime},\left.b^{\prime}\right|_{K^{\prime}}, H^{\prime}\right)$ of $\left(\Gamma^{\prime}, b^{\prime}, G^{\prime}\right)$ and a sub-metabolic form $\left(K,\left.b\right|_{K}, H\right)$ of $\left(\Gamma, b, G^{\prime}\right)$ such that

$$
\left(K^{\prime},\left.b\right|_{K^{\prime}}, H^{\prime}\right) \cong\left(K,\left.b\right|_{K}, H\right) .
$$

Thus, $\left(K^{\perp},\left.b^{\prime}\right|_{K^{\prime \perp}}\right)$ and $\left(K^{\perp},\left.b\right|_{K^{\perp}}\right)$ are equivalent bilinear forms. By induction assumption $\left(K^{\prime},\left.b\right|_{K^{\prime}}, H^{\prime}\right)^{\perp} \cong\left(K,\left.b\right|_{K}, H\right)^{\perp}$ and hence

$$
\begin{aligned}
\left(\Gamma^{\prime}, b^{\prime}, G,\right) & \cong\left(K^{\prime},\left.b\right|_{K^{\prime}}, H^{\prime}\right) \perp\left(K^{\prime},\left.b\right|_{K^{\prime}}, H^{\prime}\right)^{\perp} \\
& \cong\left(K,\left.b\right|_{K}, H\right) \perp\left(K,\left.b\right|_{K}, H\right)^{\perp} \cong(\Gamma, b, G) .
\end{aligned}
$$

Theorem 13.8. Let $p$ be an odd prime with $G$ isomorphic to $\left(\mathbb{Z}_{p^{n}}\right)^{k}$.

(i) For any $\omega \in Z^{3}\left(G, \mathbb{C}^{*}\right)_{a b}$, there exists $\eta_{1}, \ldots, \eta_{k} \in Z^{3}\left(\mathbb{Z}_{p^{n}}, \mathbb{C}^{*}\right)_{a b}$ such that $D^{\omega}(G)$ is gauge equivalent to $\bigotimes_{i=1}^{k} D^{\eta_{i}}\left(\mathbb{Z}_{p^{n}}\right)$ as quasi-triangular quasi-bialgebras.

(ii) For any $\omega, \omega^{\prime} \in Z^{3}\left(G, \mathbb{C}^{*}\right)_{a b}, D^{\omega}(G)$ and $D^{\omega^{\prime}}(G)$ are gauge equivalent as quasi-triangular quasi-bialgebras if, and only if, there exists $\rho \in \operatorname{Aut}(G)$ such that $[\widehat{\rho} \omega]=\left[\omega^{\prime}\right]$.

Proof. (i) Consider a metabolic form $\left(\Gamma^{\omega}, b_{\omega}, \widehat{G}\right)$ associated to $\omega$. By Theorem 13.7 $\left(\Gamma^{\omega}, b_{\omega}, \widehat{G}\right)$ is equivalent to the orthogonal sum

$$
\left(\Gamma_{1}, b_{1}, \mathbb{Z}_{p^{n}}\right) \perp \cdots \perp\left(\Gamma_{k}, b_{k}, \mathbb{Z}_{p^{n}}\right) .
$$

By Theorem 11.3 (iv), there exist $\eta_{i} \in Z^{3}\left(\mathbb{Z}_{p^{n}}, \mathbb{C}^{*}\right)$ such that $\left(\Gamma_{i}, b_{i}\right)$ is equivalent to $\left(\Gamma^{\eta_{i}}, b_{\eta_{i}}\right)$. Hence, by equation (69), the result follows.

(ii) If $D^{\omega}(G)$ and $D^{\omega^{\prime}}(G)$ are equivalent as quasi-triangular quasi-bialgebras, by Theorem 10.4, $\left(\Gamma^{\omega}, b_{\omega}\right)$ and $\left(\Gamma^{\omega^{\prime}}, b_{\omega^{\prime}}\right)$ are equivalent bilinear forms. It follows from Theorem 13.7(ii) that $\left(\Gamma^{\omega}, b_{\omega}, \widehat{G}\right)$ and $\left(\Gamma^{\omega^{\prime}}, b_{\omega^{\prime}}, \widehat{G}\right)$ are equivalent metabolic forms. By Proposition 13.1, $[\hat{\rho} \omega]=\left[\omega^{\prime}\right]$ for some $\rho \in \operatorname{Aut}(G)$. The "only if" part follows from Remark 2.1(iii).

\section{Duality AND Symmetry}

In this section we discuss various kinds of relations that exist between suitably chosen $D^{\omega}(G)$ and $D^{\omega^{\prime}}\left(G^{\prime}\right)$. Our discussion is meant to illustrate the possibilities, and is by no means exhaustive. We make use of Wall's classification of symmetric bilinear forms [Wal63] as well as results previously established in the present paper.

The first type of relation that we discuss-symmetry-arises from the possibility that a quadratic space $(\Gamma, q)$ may have metabolizers $G, G^{\prime}$ which are not conjugate 
in the corresponding orthogonal group. Indeed, they may not even be isomorphic. This is, in fact, a rather common phenomena.

As an example, take $\Gamma \cong \mathbb{Z}_{p^{2}} \times \mathbb{Z}_{p^{2}}$ (and for convenience, $p$ an odd prime). By Wal63, the possible non-degenerate bilinear forms on $\Gamma$ can be taken to be the following: $A_{p^{2}} \oplus A_{p^{2}}, A_{p^{2}} \oplus B_{p^{2}}$. Of these, only one has a (necessarily split) cyclic metabolizer: the first if $p \equiv 1(\bmod 4)$ and the second if $p \equiv 3(\bmod 4)$. On the other hand, the subgroup of $\Gamma$ consisting of elements of order at most $p$ is a metabolizer in all cases. As we know, each of these metabolizers $G$ determines an abelian 3-cocycle $\omega \in Z^{3}\left(G, \mathbb{C}^{*}\right)_{a b}$ such that $\Gamma^{\omega} \cong \Gamma$, and $\omega$ is even a coboundary if $G$ is cyclic. More is true: if they determine the same pair $(\Gamma, q)$, then the corresponding module categories are braided monoidally equivalent. We thus conclude:

Example 14.1. Let $p$ be an odd prime, $G \cong \mathbb{Z}_{p^{2}}, G^{\prime} \cong \mathbb{Z}_{p} \times \mathbb{Z}_{p}$. Then there is an abelian 3-cocycle $\omega^{\prime} \in Z^{3}\left(G^{\prime}, \mathbb{C}^{*}\right)_{a b}$ such that $D(G)$-Mod and $D^{\omega^{\prime}}\left(G^{\prime}\right)$-Mod are equivalent as braided tensor categories. The same argument applies if we take $G^{\prime} \cong \mathbb{Z}_{p^{n}} \times \mathbb{Z}_{p^{n}}$ and $G \cong \mathbb{Z}_{p^{2 n}}$, and to a host of other situations.

Next we discuss a duality between the module categories corresponding to the twisted double of homogeneous $p$-groups.

Theorem 14.2. Let $n, k$ be a pair of positive integers, and let $G \cong\left(\mathbb{Z}_{p^{n}}\right)^{k}$ and $G^{\prime} \cong\left(\mathbb{Z}_{p^{k}}\right)^{n}$ (p an odd prime). Then the following hold:

(i) There are exactly $\left(\begin{array}{c}n+k \\ k\end{array}\right)$ equivalence classes of monoidal categories of the form $D^{\omega}(G)$-Mod, for some $\omega \in Z^{3}\left(G, \mathbb{C}^{*}\right)_{a b}$.

(ii) There is a canonical bijection between equivalence classes of monoidal categories of the form $D^{\omega}(G)$-Mod and those of the form $D^{\omega^{\prime}}\left(G^{\prime}\right)$-Mod.

(iii) There are natural bijections between equivalence classes of braided monoidal categories of the form $D^{\omega}(G)$-Mod and those of the form $D^{\omega^{\prime}}\left(G^{\prime}\right)$-Mod.

Example 14.3. One can also count the number of braided monoidal categories (up to equivalence) of the form $D^{\omega}(G)$-Mod, where $G=\left(\mathbb{Z}_{p^{n}}\right)^{k}$ and $\omega \in Z^{3}\left(G, \mathbb{C}^{*}\right)_{a b}$. Thus we have

\begin{tabular}{c||c}
$k$ & $\#$ braided monoidal categories \\
\hline 1 & $(2 n+1)$ \\
2 & $2 n^{2}+2 n+1$ \\
3 & $1 / 3\left(4 n^{3}+6 n^{2}+8 n+3\right)$ \\
$\ldots$ & $\cdots$
\end{tabular}

To prove these assertions, recall from Theorem 13.7 that if $G=\left(\mathbb{Z}_{p^{n}}\right)^{k}$ is homogeneous, $p$ odd, then the pair $(G, \omega), \omega \in Z^{3}\left(G, \mathbb{C}^{*}\right)_{a b}$, determines a non-degenerate bilinear form $(\Gamma, b)$ of the form

$$
(\Gamma, b)=\left(H_{1}, b_{1}\right) \perp \cdots \perp\left(H_{k}, b_{k}\right)
$$

where $\left(H_{i}, b_{i}\right)$ has a cyclic metabolizer $G_{i}, G=G_{1} \times \cdots \times G_{k}$, and $H_{i}$ is isomorphic to $\mathbb{Z}_{p^{n+a}} \oplus \mathbb{Z}_{p^{n-a}}$ for some integer $a$ with $0 \leq a \leq n$. Of course, $\Gamma$ is isomorphic to the group of fusion rules $\Gamma^{\omega}$. Denote by $\Gamma_{a}$ the group $\mathbb{Z}_{p^{n+a}} \oplus \mathbb{Z}_{p^{n-a}}$ (where now $n$ is fixed). If $\Gamma_{a}$ occurs with multiplicity $m_{a}$ in (70), we can represent (70) symbolically in the form

$$
\Gamma=\perp_{a=0}^{n} m_{a} \Gamma_{a}
$$


at least as far as the fusion rules are concerned. It is easy to see that we have

$$
\sum_{a=0}^{n} m_{a}=k
$$

Furthermore, any $n+1$-tuple $\left(m_{0}, m_{1}, \ldots, m_{n}\right)$ of nonnegative integers satisfying (72) may be chosen in (71) and which correspond to a pair $(\Gamma, b)$ with metabolizer $G$. Thus the number of nonisomorphic groups of fusion rules $\Gamma$ that correspond to $G$ is the number of nonnegative $(n+1)$-tuples $\left(m_{0}, m_{1}, \ldots, m_{n}\right)$ satisfying (72). This is easily seen to equal $\left(\begin{array}{c}n+k \\ k\end{array}\right)$. Now part (i) of the theorem follows from Theorem 9.4.

Note that if also $G^{\prime}=\left(\mathbb{Z}_{p^{k}}\right)^{n}$, then by part (i), there are $\left(\begin{array}{c}n+k \\ k\end{array}\right)$ equivalence classes of tensor categories both of the type $D^{\omega}(G)$-Mod and $D^{\omega^{\prime}}\left(G^{\prime}\right)$-Mod. We will establish a canonical bijection between these two sets.

With $\left(m_{0}, m_{1}, \ldots, m_{n}\right)$ as above, let $\lambda$ be the partition which contains the integer $i$ with multiplicity $m_{i}, 1 \leq i \leq n$. Thus $\lambda$ is a partition of the integer $r=\sum_{i=1}^{n} i m_{i}$. Note that $r \leq n k$ by (72). Let $\lambda^{t}$ be the dual (conjugate) partition of $\lambda$, and let $s_{j}$ be the multiplicity of $j$ in $\lambda^{t}$ for $j \geq 1$. So also $r=\sum_{j=1}^{k} j s_{j}$, the upper limit $k$ in the sum arising from the fact that the maximal part of $\lambda^{t}$ (i.e., the largest $j$ with $\left.s_{i}>0\right)$ is equal to the number of non-zero multiplicities $m_{i}$, and this is at most $k$ by (72).

Now observe that $\sum_{j=1}^{k} s_{j}=\#$ of parts of the partition $\lambda^{t}=\max \left\{i \mid m_{i}>0\right\} \leq n$. Thus if we define $s_{0}=n-\sum_{j=1}^{k} s_{j}$, then $s_{j} \geq 0$ for $j \geq 0$ and

$$
\sum_{j=0}^{k} s_{j}=n \text {. }
$$

Consider

$$
\Gamma^{\prime}=\perp_{j=0}^{k} s_{j} \Gamma_{j}^{\prime}
$$

where $\Gamma_{j}^{\prime}=\mathbb{Z}_{p^{k+j}} \oplus \mathbb{Z}_{p^{k-j}}$. By previous arguments, $\Gamma^{\prime}$ is the group of fusion rules corresponding to a suitable pair $\left(G^{\prime}, \omega^{\prime}\right)$. Thus (73) and (74) enjoy the same relation to $\left(G^{\prime}, \omega^{\prime}\right)$ that (71) and (72) do to $(G, \omega)$.

Thus the canonical bijection (duality) which relates $\Gamma$ and $\Gamma^{\prime}$ is achieved via the duality between the partitions $\lambda$ and $\lambda^{t}$ which are associated to them. This explains part (ii) of the theorem.

Example 14.4. In Table 1 below, we illustrate this duality in case the two groups in question are $G=\mathbb{Z}_{p^{2}} \times \mathbb{Z}_{p^{2}} \times \mathbb{Z}_{p^{2}}$ and $G^{\prime}=\mathbb{Z}_{p^{3}} \times \mathbb{Z}_{p^{3}}$. Groups $\Gamma, \Gamma^{\prime}$ on the same line are in duality.

Turning to part (iii), we first establish:

Lemma 14.5. With the notation of (71), let $f$ be the number of indices $a \geq 1$ such that $m_{a} \geq 1$. Then the number of inequivalent, non-degenerate bilinear forms $b$ on $\Gamma$ with metabolizer (isomorphic to) $G$ is $2^{f}$.

Proof. From Theorem 13.7 we only need to enumerate the equivalence classes of bilinear forms that arise as in (70) and (71) above.

To show that this number is $2^{f}$, let us assume for convenience that $p \equiv 1$ $(\bmod 4)($ the case $p \equiv 3(\bmod 4)$ is proved similarly). According to Wal63 and the discussion following equation (65), the only forms $\Gamma_{a}$ with cyclic metabolizer 
TABLE 1.

\begin{tabular}{|c|c|c|c|c|}
\hline \multicolumn{2}{|r|}{$G=\mathbb{Z}_{p^{2}} \times \mathbb{Z}_{p^{2}} \times \mathbb{Z}_{p^{2}}$} & \multicolumn{2}{|c|}{$G^{\prime}=\mathbb{Z}_{p^{3}} \times \mathbb{Z}_{p^{3}}$} & \\
\hline$\left(m_{1}, m_{2}\right)$ & $\Gamma$ & $\left(s_{1}, s_{2}, s_{3}\right)$ & $\Gamma^{\prime}$ & $f$ \\
\hline$\overline{(0,3)}$ & $\overline{\mathbb{Z}_{p^{4}} \times \mathbb{Z}_{p^{4}} \times \mathbb{Z}_{p^{4}}}$ & $\overline{(0,0,2)}$ & $\overline{\mathbb{Z}_{p^{6}} \times \mathbb{Z}_{p^{6}}}$ & $\overline{1} 1$ \\
\hline$(1,2)$ & $\mathbb{Z}_{p^{4}} \times \mathbb{Z}_{p^{4}} \times \mathbb{Z}_{p^{3}} \times \mathbb{Z}_{p}$ & $(0,1,1)$ & $\mathbb{Z}_{p^{6}} \times \mathbb{Z}_{p^{5}} \times \mathbb{Z}_{p}$ & 2 \\
\hline$(0,2)$ & $\mathbb{Z}_{p^{4}} \times \mathbb{Z}_{p^{4}} \times \mathbb{Z}_{p^{2}} \times \mathbb{Z}_{p^{2}}$ & $(0,2,0)$ & $\mathbb{Z}_{p^{5}} \times \mathbb{Z}_{p^{5}} \times \mathbb{Z}_{p} \times \mathbb{Z}_{p}$ & 1 \\
\hline$(2,1)$ & $\mathbb{Z}_{p^{4}} \times \mathbb{Z}_{p^{3}} \times \mathbb{Z}_{p^{3}} \times \mathbb{Z}_{p} \times \mathbb{Z}_{p}$ & $(1,0,1)$ & $\mathbb{Z}_{p^{6}} \times \mathbb{Z}_{p^{4}} \times \mathbb{Z}_{p^{2}}$ & 2 \\
\hline$(1,1)$ & $\mathbb{Z}_{p^{4}} \times \mathbb{Z}_{p^{3}} \times \mathbb{Z}_{p^{2}} \times \mathbb{Z}_{p^{2}} \times \mathbb{Z}_{p}$ & $(1,1,0)$ & $\mathbb{Z}_{p^{5}} \times \mathbb{Z}_{p^{4}} \times \mathbb{Z}_{p^{2}} \times \mathbb{Z}_{p}$ & 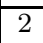 \\
\hline$(0,1)$ & $\frac{p}{\mathbb{Z}_{p^{4}} \times \mathbb{Z}_{p^{2}} \times \mathbb{Z}_{p^{2}} \times \mathbb{Z}_{p^{2}} \times \mathbb{Z}_{p^{2}}}$ & $(0,2,0)$ & $\stackrel{p}{\mathbb{Z}_{p^{4}} \times \mathbb{Z}_{p^{4}} \times \mathbb{Z}_{p^{2}} \times \mathbb{Z}_{p^{2}}}$ & 1 \\
\hline$(2,0)$ & $\mathbb{Z}_{p^{3}} \times \mathbb{Z}_{p^{3}} \times \mathbb{Z}_{p^{2}} \times \mathbb{Z}_{p^{2}} \times \mathbb{Z}_{p} \times \mathbb{Z}_{p}$ & $(0,1,0)$ & $\mathbb{Z}_{p^{5}} \times \mathbb{Z}_{p^{3}} \times \mathbb{Z}_{p^{3}} \times \mathbb{Z}_{p}$ & \\
\hline$(3,0)$ & 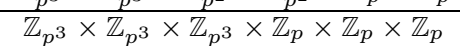 & $(0,0,1)$ & $\mathbb{Z}_{p^{6}} \times \mathbb{Z}_{p^{3}} \times \mathbb{Z}_{p^{3}}$ & \\
\hline$(1,0)$ & $\mathbb{Z}_{p^{3}} \times \mathbb{Z}_{p^{2}} \times \mathbb{Z}_{p^{2}} \times \mathbb{Z}_{p^{2}} \times \mathbb{Z}_{p^{2}} \times \mathbb{Z}_{p}$ & $(1,0,0)$ & $\mathbb{Z}_{p^{4}} \times \mathbb{Z}_{p^{3}} \times \mathbb{Z}_{p^{3}} \times \mathbb{Z}_{p^{2}}$ & 1 \\
\hline$(0,0)$ & $\left(\mathbb{Z}_{p^{2}}\right)$ & $(0,0,0)$ & $\left(\mathbb{Z}_{p^{3}}\right)$ & 0 \\
\hline
\end{tabular}

are of type $A \oplus A$ or type $B \oplus B$ if $1 \leq a \leq n-1$; type $A$ or type $B$ if $a=n$; type $A \oplus A$ (@ type $B \oplus B)$ if $a=0$. Furthermore, if $a \geq 1$, the only forms on $m_{a} \Gamma_{a}$ with metabolizer $\left(\mathbb{Z}_{p^{n}}\right)^{m_{a}}$ are (up to equivalence) of the shape $m_{a}(A \oplus A)$ or $\left(m_{a}-1\right)(A \oplus A) \oplus(B \oplus B)$. Thus, as long as $m_{a} \geq 1$ and $a \geq 1$, there are in any case just two inequivalent forms on $m_{a} \Gamma_{a}$ with homogeneous metabolizer $\left(\mathbb{Z}_{p^{n}}\right)^{m_{a}}$. Now the lemma follows immediately.

Note that the integer $f$ of Lemma 14.5 is the number of unequal parts of the partition $\lambda$. But it is easy to see (and well-known) that this is equal also to the number of unequal parts of $\lambda^{t}$. So the canonical bijection of part (ii) of Theorem 14.2 also induces bijections between the inequivalent braided monoidal categories of the form $D^{\omega}(G)$-Mod and $D^{\omega^{\prime}}\left(G^{\prime}\right)$-Mod which have fusion rules $\Gamma, \Gamma^{\prime}$ respectively. This holds for all such $\Gamma$, so that the number of inequivalent braided tensor categories $D^{\omega}(G)$-Mod is the same as the number of type $D^{\omega^{\prime}}\left(G^{\prime}\right)$-Mod.

This is not quite a natural bijection. However, we saw above that the allowable bilinear forms on $\Gamma$ are naturally indexed by $f$-tuples consisting of $A$ 's and $B$ 's. More precisely, let us again assume for convenience that $p \equiv 1(\bmod 4)$. If $a \geq 1$ with $m_{a} \geq 1$, then we may take all but one $\Gamma_{a}$ in (71) to be type $A$ or $A \oplus A$ (according to whether $a=n$ or $a<n$ ); the remaining $\Gamma_{a}$ may be taken to be either type $A$ or type $B$ (or type $A \oplus A$ or $B \oplus B$ ). All such $f$-tuples are allowable, so that there are exactly $2^{f}$ such tuples, as claimed. Since (by the lemma) this analysis applies not only to $\Gamma$ but to the dual group $\Gamma^{\prime}$, we obtain a "natural" bijection between bilinear forms, and hence braided monoidal categories, by associating those forms which correspond to the same $f$-tuple of $A$ 's and $B$ 's. This completes our discussion of part (iii) of the theorem.

Finally, the formulae in example 14.3 follow from our analysis without difficulty. For example, take the case $k=2$, so that $G \cong \mathbb{Z}_{p^{n}} \oplus \mathbb{Z}_{p^{n}}$. The possible $(n+1)$-tuples $\left(m_{0}, m_{1}, \ldots, m_{n}\right)$ with $\sum_{a \geq 0} m_{a}=2$ are trivially enumerated, and each gives rise to $2^{f}$ inequivalent forms as in Lemma 14.5. If $m_{0}=2$, then $f=0$; if $m_{0}=1$ or $m_{0}=0$ and $m_{a}=2$ for some $a \geq 1$, then $f=1$; otherwise $f=2$. Thus the total number of inequivalent allowable forms (or braided monoidal categories) is $1+2 n+2 n+4\left(\begin{array}{c}n \\ 2\end{array}\right)=2 n^{2}+2 n+1$.

Example 14.6. We can read off further examples of symmetry from Table 1. Consider the group $\Gamma=\mathbb{Z}_{p^{4}} \times \mathbb{Z}_{p^{4}} \times \mathbb{Z}_{p^{2}} \times \mathbb{Z}_{p^{2}}$. We see that the two groups 
$G=\mathbb{Z}_{p^{2}} \times \mathbb{Z}_{p^{2}} \times \mathbb{Z}_{p^{2}}$ and $G^{\prime}=\mathbb{Z}_{p^{3}} \times \mathbb{Z}_{p^{3}}$ each admit $\Gamma$ as the corresponding fusion rules for some choice of quadratic form. Indeed, the previous discussion shows that there are exactly two non-degenerate quadratic forms on $\Gamma$ with metabolizers equal to both $G$ and $G^{\prime}$. By Theorem 10.4 it follows that the following holds: there are cohomology classes $\left[\omega_{1}\right],\left[\omega_{2}\right]$ in $H^{3}\left(G, \mathbb{C}^{*}\right)_{a b}$ and $\left[\omega_{1}^{\prime}\right],\left[\omega_{2}^{\prime}\right]$ in $H^{3}\left(G^{\prime}, \mathbb{C}^{*}\right)_{a b}$ such that $D^{\omega_{i}}(G)$ and $D^{\omega_{i}^{\prime}}\left(G^{\prime}\right), i=1,2$, are gauge equivalent.

\section{REFERENCES}

[AF92] Frank W. Anderson and Kent R. Fuller, Rings and categories of modules, Second ed., Springer-Verlag, New York, 1992. MR 94i:16001

[Bor86] Richard E. Borcherds, Vertex algebras, Kac-Moody algebras, and the Monster, Proc. Nat. Acad. Sci. U.S.A. 83 (1986), no. 10, 3068-3071. MR 87m:17033

[Bro82] Kenneth S. Brown, Cohomology of groups, Springer-Verlag, New York-Berlin, 1982; Corrected reprint, Springer-Verlag, 1994. MR 83k:20002; MR 96a:20072

[CP95] Vyjayanthi Chari and Andrew Pressley, A guide to quantum groups, Cambridge University Press, Cambridge, 1995, Corrected reprint of the 1994 original. MR 96h:17014

[DL93] Chongying Dong and James Lepowsky, Generalized vertex algebras and relative vertex operators, Birkhäuser Boston, Inc., Boston, MA, 1993. MR 95b:17032

[DPR92] R. Dijkgraaf, V. Pasquier, and P. Roche, Quasi-Hopf algebras, group cohomology and orbifold models, Integrable systems and quantum groups (Pavia, 1990), World Sci. Publishing, River Edge, NJ, 1992, pp. 75-98. MR 92m:81238

[Dri87] V. G. Drinfel'd, Quantum groups, Proceedings of the International Congress of Mathematicians, Vol. 1, 2 (Berkeley, Calif., 1986), Amer. Math. Soc., Providence, RI, 1987, pp. 798-820. MR 89f:17017

[Dri90] V. G. Drinfel'd, Quasi-Hopf algebras, Leningrad Math. J. 1 (1990), 1419-1457. MR 91b:17016

[DW90] Robbert Dijkgraaf and Edward Witten, Topological gauge theories and group cohomology, Comm. Math. Phys. 129 (1990), no. 2, 393-429. MR 91g:81133

[EM50a] Samuel Eilenberg and Saunders Mac Lane, Cohomology theory of Abelian groups and homotopy theory. I, Proc. Nat. Acad. Sci. U. S. A. 36 (1950), 443-447. MR 12:350c

[EM50b] Samuel Eilenberg and Saunders Mac Lane, Cohomology theory of Abelian groups and homotopy theory. II, Proc. Nat. Acad. Sci. U. S. A. 36 (1950), 657-663. MR 12:520a

[FHL93] Igor B. Frenkel, Yi Zhi Huang, and James Lepowsky, On axiomatic approaches to vertex operator algebras and modules, Mem. Amer. Math. Soc. 104 (1993), no. 494, viii+64. MR 94a:17007

[FLM88] Igor Frenkel, James Lepowsky, and Arne Meurman, Vertex operator algebras and the Monster, Academic Press Inc., Boston, MA, 1988. MR 90h:17026

[HL92] Yi Zhi Huang and James Lepowsky, Toward a theory of tensor products for representations of a vertex operator algebra, Proceedings of the XXth International Conference on Differential Geometric Methods in Theoretical Physics, Vol. 1, 2 (New York, 1991) (River Edge, NJ), World Sci. Publishing, 1992, pp. 344-354. MR 94k:17045

[HL94a] Yi-Zhi Huang and James Lepowsky, Tensor products of modules for a vertex operator algebra and vertex tensor categories, Lie theory and geometry, Birkhäuser Boston, Boston, MA, 1994, pp. 349-383. MR 96e:17061

[HL94b] Yi-Zhi Huang and James Lepowsky, A theory of tensor products for module categories for a vertex operator algebra. I, Sūrikaisekikenkyūsho Kōkyūroku (1994), no. 883, 148203, Geometric aspects of infinite integrable systems (Japanese) (Kyoto, 1993). MR 98a: 17048

[HL95a] Y.-Z. Huang and J. Lepowsky, A theory of tensor products for module categories for a vertex operator algebra. I, II, Selecta Math. (N.S.) 1 (1995), no. 4, 699-756, 757-786. MR 98a:17047

[HL95b] Yi-Zhi Huang and James Lepowsky, A theory of tensor products for module categories for a vertex operator algebra. III, J. Pure Appl. Algebra 100 (1995), no. 1-3, 141-171. MR 98a:17049

[HO97] Reinhard Häring-Oldenburg, Reconstruction of weak quasi Hopf algebras, J. Algebra 194 (1997), no. 1, 14-35. MR 98h:16062 
[JS93] André Joyal and Ross Street, Braided tensor categories, Adv. Math. 102 (1993), no. 1, 20-78. MR 94m:18008

[Kas95] Christian Kassel, Quantum groups, Springer-Verlag, New York, 1995. MR 96e:17041

[Kne70] Manfred Knebusch, Grothendieck- und Wittringe von nichtausgearteten symmetrischen Bilinearformen, S.-B. Heidelberger Akad. Wiss. Math.-Natur. Kl. 1969/70 (1969/1970), 93-157. MR 42:6001

[Lam99] T. Y. Lam, Lectures on modules and rings, Springer-Verlag, New York, 1999. MR 99i: 16001

[Mac52] Saunders Mac Lane, Cohomology theory of Abelian groups, Proceedings of the International Congress of Mathematicians, Cambridge, Mass., 1950, vol. 2, Amer. Math. Soc., Providence, R. I., 1952, pp. 8-14. MR 13:529d

[Maj92] Shahn Majid, Tannaka-Krein theorem for quasi-Hopf algebras and other results, Deformation theory and quantum groups with applications to mathematical physics (Amherst, MA, 1990), Amer. Math. Soc., Providence, RI, 1992, pp. 219-232. MR 93k:16073

[Mas95] Geoffrey Mason, The quantum double of a finite group and its role in conformal field theory, Groups '93 Galway/St. Andrews, Vol. 2, Cambridge Univ. Press, Cambridge, 1995, pp. 405-417. MR 97a:11067

[MH73] John Milnor and Dale Husemoller, Symmetric bilinear forms, Springer-Verlag, New York, 1973, Ergebnisse der Mathematik und ihrer Grenzgebiete, Band 73. MR 58:22129

[MS89] Gregory Moore and Nathan Seiberg, Classical and quantum conformal field theory, Comm. Math. Phys. 123 (1989), no. 2, 177-254. MR 90e:81216

[Swe69] Moss E. Sweedler, Hopf algebras, W. A. Benjamin, Inc., New York, 1969, Mathematics Lecture Note Series. MR 40:5705

[tD79] Tammo tom Dieck, Transformation groups and representation theory, Springer, Berlin, 1979. MR 82c:57025

[TY98] Daisuke Tambara and Shigeru Yamagami, Tensor categories with fusion rules of self-duality for finite abelian groups, J. Algebra 209 (1998), no. 2, 692-707. MR 2000b: 18013

[Wal63] C. T. C. Wall, Quadratic forms on finite groups, and related topics, Topology 2 (1963), 281-298. MR 28:133

Department of Mathematics, University of California, Santa Cruz, California 95064

E-mail address: gem@cats.ucsc.edu

Department of Mathematics, University of California, Santa Cruz, California 95064

Current address: Department of Mathematics, Towson University, Baltimore, Maryland 21252

E-mail address: rng@towson.edu 